\title{
Estabilidade Não Linear de Equações a Derivadas Parciais do Tipo Parabólico
}

\author{
José Eduardo Castilho
}

Orientação:

José Alberto Cuminato

Dissertação apresentada ao Instituto de Ciências Matemáticas de São Carlos, da Universidade de São Paulo, como parte dos requisitos para obtenção do título de mestre em Ciências de Computação e Matemática Computacional.

USP - São Carlos

1.991 
Dedico este trabalho

Aos meus pais e a Tina,

pelo apoio, dedicação e incentivo constantes. 


\section{Agradecimentos}

Ao Poti, pela orientação sempre segura, dedicada e objetiva.

Ao Prof. Odelar, pelas constantes palavras de incentivo.

Ao Mauro, Ben-Hur e Tereza Cristina, pela amizade e companheirismo durante todas as atividades dentro e fora do ICMSC-USP.

Aos professores e funcionários do ICMSC-USP, pela atenção que me foi dedicada.

À CAPES pelo apoio financeiro.

À todos os trabalhadores deste país.

Àqueles que de um modo ou de outro contribuiram para a realização deste trabalho. 


\begin{abstract}
The main purpose of this work is to describe the manisfetation of numerical instability in Reaction-Diffusion problems.

A unified analysis of the continuous problem and its discretisation shows clearly when and why the discretisation breaks down. This analysis provides background for interpretation of numerical instability in nonlinear parabolic partial differential equations.

The problems, continuous and discrete, are analysed from the points of view of local bifurcation, linear stability and weakly nonlinear stability theories. It is shown that numerical instability is associated with the bifurcation of periodic orbits in the discrete problems, a fact that does not happen in the continuous case. Numerical examples that illustrate the various possibilities are presented and analysed in light of this theory.
\end{abstract}




\section{Resumo}

O objetivo principal deste trabalho é descrever a manifestação da instabilidade numérica em problemas de Reação-Difusão.

Uma análise conjunta do problema contínuo e sua discretização mostra claramente onde e quando a discretização falha. Esta análise fornece um conhecimento básico para a interpretação da instabilidade numérica em equações diferenciais parciais parabólicas não lineares.

Os problemas, contínuo e discreto, são analisados através da teoria da bifurcação local, estabilidade linear e estabilidade não linear fraca.

Mostra-se que a instabilidade numérica está associada com a bifurcação periódica no problema discreto, fato que não ocorre no problema contínuo. Isto é ilustrado através de exemplos numéricos. 


\section{Conteúdo}

1 Introdução às Equações Diferenciais Parciais Parabólicas 1

1.1 Definições Básicas . . . . . . . . . . . . . . . . 1

1.2 Um exemplo de EDP Parabólica . . . . . . . . . . . . . . . . 2

1.3 Método de Separação de Variáveis . . . . . . . . . . . . 3

1.4 Princípio do Máximo-Mínimo ............. 5

2 Métodos de Diferenças Finitas $\quad 7$

2.1 Aproximação de Derivadas por Diferenças Finitas . . . . . . . 7

2.2 Um Método Explícito . . . . . . . . . . . . . . . . 11

2.3 Métodos de Crank-Nicolson ............... . 12

2.4 Condições de Fronteira a Derivadas . . . . . . . . . . . . 13

2.5 Erro de Truncamento Local . . . . . . . . . . . . . . . 14

2.6 Cońsistência ou Compatibilidade ............ 15

2.7 Convergência ....................... 16

2.8 Estabilidade Absoluta . . . . . . . . . . . . 18

2.9 Equação Não Linear . . . . . . . . . . . . . . . . . . 22

3 Equação Reação-Difusão 25

3.1 Introdução à Teoria da Bifurcação . . . . . . . . . . . . 25

3.2 Problema de Reação-Difusão . . . . . . . . . . . . . . . . . 29

3.3 O Problema Linearizado . . . . . . . . . . . . . . 30 
3.4 Análise das Bifurcações . . . . . . . . . . . . . . . . 31

3.5 Análise Não Linear Fraca . . . . . . . . . . . . . . . . . . 34

3.6 Bifurcação no Infinito . . . . . . . . . . . . . . 39

4 Discretização da Equação Reação-Difusão 40

4.1 O Problema Discreto . . . . . . . . . . . . . . 40

4.2 O Problema Linearizado . . . . . . . . . . . . . 41

4.3 Análise das Bifurcações . . . . . . . . . . . . . . . . . . 42

4.4 Análise Não Linear Fraca . . . . . . . . . . . . . . . . 46

4.5 Bifurcação no Infinito . . . . . . . . . . . . . . . . 64

5 Conclusão 65

$\begin{array}{ll}\text { Bibliografia } & 67\end{array}$ 


\section{Capítulo 1}

\section{Introdução às Equações Diferenciais Parciais Parabólicas}

Neste capítulo procuramos dar uma noção geral do problema de equações diferenciais parciais parabólicas. Inicialmente damos algumas definições básicas quanto aos tipos de equações, das condições iniciais e de contorno.

Consideramos o problema da condução do calor em uma barra como exemplo de equação parabólica e procuramos condições de existência pelo Método de Separação de Variáveis, unicidade e dependência contínua dos dados iniciais pelo Princípio do Máximo-Mínimo.

\subsection{Definições Básicas}

Com a criação do Cálculo Diferencial e Integral no século XVII as equações diferenciais parciais surgiram da procura de modelos matemáticos que descrevessem os fenômenos da Mecânica do Contínuo e outros ramos da Física.

A forma geral de uma equação diferencial parcial (EDP) de segunda ordem em duas variáveis independentes é a seguinte:

$$
a u_{x x}+2 b u_{x t}+c u_{t t}+d u_{x}+e u_{t}+f u+g=0 .
$$

Quando algum dos coeficientes $a, b, \ldots, g$ for uma função de $u$ ou de alguma derivada de $u$ dizemos que a equação é não linear, caso contrário será linear.

As curvas ao longo das quais a EDP pode ser escrita, numa forma con- 
tendo apenas derivadas totais de $u_{x}$ e $u_{t}$, são conhecidas como curvas características, ver Willians [25], e estas curvas satisfazem a equação:

$$
a\left(\frac{d x}{d t}\right)^{2}-2 b \frac{d x}{d t}+c=0
$$

De acordo com o sinal do discriminante $\Delta=b^{2}-a c$ existirá uma, duas ou nenhuma curva característica da equação (1.1). Com isto introduzimos a seguinte definição:

Definição 1.1.1: A equação (1.1) é dita ser do tipo:

(i ) Elíptica quando $\Delta<0$;

(ii) Parabólica quando $\Delta=0$;

(iii) Hiperbólica quando $\Delta>0$.

Assim como em EDO's as EDP's necessitam de informações suplementares para a unicidade de solução. No caso das EDP's temos que a solução está definida numa região $\Omega \subseteq \mathbf{R}^{n}$. Quando impomos condições sobre a fronteira da região $\Omega$ temos um problema de contorno e quando as condições são dadas numa sub-variedade de co-dimensão um em $\mathbf{R}^{n}$ temos o problema de valor inicial. Em certos problemas, como o fenômeno de difusão, temos que impor condições de contorno e condições iniciais para obtermos a solução, um exemplo é a equação da condução do calor.

\section{2 Ưm exemplo de EDP Parabólica}

Consideremos o problema de variação de temperatura de uma barra de secção reta uniforme e comprimento $L$, feita de material homogêneo com difusividade térmica $\alpha^{2}$ satisfazendo as seguintes hipóteses:

(i ) A dimensão da secção reta é pequena em relação ao comprimento L;

(ii) A superfície lateral da barra é isolada termicamente;

(iii) A temperatura nas extremidades da barra é mantida constante com valor igual a zero ( condição de contorno); 
(iv) No instante $t=0$ a barra tem uma distribuição de temperatura $u_{0}(x)$ conhecida (condição inicial).

Este fenômeno físico pode ser representado pelo problema em EDP dado por:

$$
\begin{cases}u_{t}=\alpha^{2} u_{x x} ; & x \in(0, L), t>0 \\ u(0, t)=u(L, t)=0 ; & t>0 \\ u(x, 0)=u_{0}(x) ; & x \in[0, L]\end{cases}
$$

Tendo em vista a interpretação física do problema, é natural procurarmos solução na região $\Omega=(0, L) \times(0, \infty)$ de tal forma que $u \in C^{2}(\Omega) \cap C(\bar{\Omega})$, e neste caso devemos impor que $u_{0} \in C[0, L]$. Notamos que as condições iniciais e de contorno não são independentes, pois $u_{0}(x)$ deve satisfazer as condições de compatibilidade $u_{0}(0)=u_{0}(L)=0$.

Três questões são fundamentais, existência, unicidade e dependência contínua dos dados iniciais. Quando um problema satisfaz estas três propriedades, dizemos que ele é bem posto no sentido de Hadamard. A existência é mostrada através do Método de Separação de Variáveis, a unicidade e a dependência contínua dos dados iniciais, através do Princípio do Máximo-Mínimo.

A discussão da dependência contínua dos dados iniciais é muito importante, pois devemos lembrar que os dados de um problema prático são experimentais, sujeitos a erros de medida. Portanto é natural perguntar se pequenas variações nos dados acarretam pequenas variações na solução. Do ponto de vista matemático isto se traduz em perguntar se as soluções variam continuamente como função dos dados iniciais em alguma topologia conveniente.

No caso das EDP's lineares, obtida a existência e a unicidade, a dependência contínua dos dados está essencialmente provada. No caso de equações não lineares a discusão é bem diferente e pode ser bastante difícil.

\subsection{Método de Separação de Variáveis}

Vamos procurar uma solução para o problema (1.2) que seja da forma $u(x, t)=\phi(x) \psi(t)$ onde:

$$
\phi(x) \in C^{2}(0, L) \cap C[0, L] \text { e } \psi(t) \in C^{2}(0, \infty) \cap C[0, \infty) .
$$

Impondo as condições de contorno temos $\phi(0) \psi(t)=\phi(L) \psi(t)=0$. Para obtermos uma solução $u(x, t)$, que não seja a solução nula, devemos ter:

$$
\phi(0)=\phi(L)=0 \text {. }
$$


Substituindo $\phi(x) \psi(t)$ na equação (1.2) e levando em conta que $x$ e $t$ são variáveis independentes temos:

$$
\frac{\phi^{\prime \prime}(x)}{\phi(x)}=-\sigma=\frac{\psi^{\prime}(t)}{\psi(t) \alpha^{2}} .
$$

Desta equação obtemos as seguintes equações diferenciais ordinárias:

$$
\begin{gathered}
\phi^{\prime \prime}(x)=-\sigma \phi(x) ; \quad 0<x<L, \\
\psi^{\prime}(t)=-\sigma \alpha^{2} \psi(t) ; \quad t>0 .
\end{gathered}
$$

0 problema (1.3)- (1.4) é um problema de autovalor para o operador $-\partial^{2} / \partial x^{2}$ com condições de contorno de Dirichlet. Não é difícil mostrar que $\sigma$ é um número real e $\sigma>0$, (ver lório [9]), logo a solução geral de (1.4) é da forma:

$$
\phi(x)=A \cos (\sqrt{\sigma} x)+B \operatorname{sen}(\sqrt{\sigma} x) .
$$

Impondo as condições de (1.3) obtemos que $\sigma=\frac{s^{2} \pi^{2}}{L^{2}}$ e conseqüentemente (1.4) e (1.5) têm como solução respectivamente:

$$
\phi_{s}(x)=B \operatorname{sen}\left(\frac{s \pi x}{L}\right) \text { e } \psi_{s}(t)=C \exp \left(\frac{-s^{2} \pi^{2}}{L^{2}} \alpha^{2} t\right),
$$

com $s$ inteiro não nulo.

Sem perda de generalidade vamos considerar $B=C=1$. Observe que o problema (1.2) é um problema linear e homogêneo, portanto vale o princípio da superposição, isto é, sendo $u_{s}(x, t)=\phi_{s}(x) \psi_{s}(t)$, então:

$$
u(x, t)=\sum_{s=1}^{\infty} b_{s} u_{s}(x, t)
$$

também é solução. Note que a variação de $s$ no somatório é restrita a valores positivos devido ao fato que $\operatorname{sen}(-s x)=-\operatorname{sen}(s x)$. Logo um candidato à solução geral de (1.2) é dado por:

$$
u(x, t)=\sum_{s=1}^{\infty} b_{s} \operatorname{sen}\left(\frac{s \pi x}{L}\right) \exp \left(\frac{-s^{2} \pi^{2}}{L^{2}} \alpha^{2} t\right) .
$$

Para determinar os coeficientes $b_{s}$ impomos a condição inicial e obtemos:

$$
u_{0}(x)=\sum_{s=1}^{\infty} b_{s} \operatorname{sen}\left(\frac{s \pi x}{L}\right)
$$

De acordo com os resultados da Análise de Fourier sabemos que quando $u_{0}(x) \in$ $C[0, L]$ e $u_{0}(0)=u_{0}(L)=0$, a série acima é convergente, e os $b_{s}$ são os coeficientes de Fourier dados por:

$$
b_{s}=\frac{2}{L} \int_{0}^{L} u_{0}(x) \phi_{s}(x) d x
$$


Sendo $u_{0}(x) \in C[0, L]$, temos da equação acima que:

$$
\left|b_{s}\right| \leq 2 \sup _{x \in[0, L]}\left|u_{0}(x)\right| ; s=0,1,2, \ldots
$$

de modo que a série (1.6) converge absoluta e uniformemente em qualquer conjunto da forma $[0, L] \times\left[t_{0}, \infty\right)$ com $t_{0}>0$. Portanto (1.6) é de fato uma solução geral de (1.2), e isto justifica a existência de soluçôes.

\subsection{Princípio do Máximo-Mínimo}

Teorema 1.4.1 (Princípio do Máximo-Mínimo): Seja $u(x, t)$ uma função contínua no retângulo $\Re=\left\{(x, t): x_{1} \leq x \leq x_{2}, t_{1} \leq t \leq \infty\right\}$, no qual $u(x, t)$ é solução de $u_{t}=\alpha^{2} u_{x x}$, então para $(x, t) \in \Re \circ \max u(x, t)(\min u(x, t))$ em $\Re$ pertence a fronteira de $\Re$.

Prova: ver Figueiredo [5] pag. 120.

Com o Princípio do Máximo-Mínimo podemos mostrar a unicidade da solução do problema (1.2). De fato:

Teorema 1.4.2 (Unicidade): Seja $u(x, t)$ uma solução para (1.2) em $\Omega=$ $[0, L] \times[0, \infty)$, então $u(x, t)$ é única.

Prova: Supondo que existam duas soluções $u_{1}(x, t)$ e $u_{2}(x, t)$ com as mesmas condiçóes iniciais e de contorno, então $w(x, t)=u_{1}(x, t)-u_{2}(x, t)$ é solução do problema:

$$
\begin{cases}w_{t}=\alpha^{2} w_{x x} ; & x \in(0, L) ; t>0 \\ w(0, t)=w(L, t)=0 ; & t>0 \\ w(x, 0)=0 ; & x \in[0, L]\end{cases}
$$

Usando o Principio do Máximo-Mínimo temos que $w(x, t) \equiv 0$, logo $u_{1}(x, t) \equiv u_{2}(x, t)$.

Teorema 1.4.3 (Dependência Contínua dos Dados): Seja $u(x, t)$ solução de (1.2) em $\Omega=[0, L] \times[0, \infty)$, então $u(x, t)$ depende continuamente dos dados iniciais.

Prova: Supondo que existam duas soluções $u_{1}(x, t)$ e $u_{2}(x, t)$ satisfazendo as condições do teorema com condições iniciais $u_{1}(x, 0)=f_{1}(x)$ e $u_{2}(x, 0)=$ 
$f_{2}(x)$, então $u(x, t)=u_{1}(x, t)-u_{2}(x, t)$ satisfaz a equação diferencial com condição inicial $u(x, 0)=f_{1}(x)-f_{2}(x)$.

Assim pelo Princípio do Máximo-Mínimo temos que:

$$
\max |u| \leq \max \left|f_{1}-f_{2}\right|
$$

o que prova o teorema. 


\section{Capítulo 2}

\section{Métodos de Diferenças Finitas}

Neste capítulo apresentamos os métodos clássicos de aproximação para equações parabólicas, usando a equação do calor como exemplo.

Iniciamos com a aproximação de derivadas por operadores de diferenças finitas. Em seguida mostramos um método explícito, passando para os métodos de Crank-Nicolson.

Estudamos também o problema de condições de fronteira com derivadas, o erro de truncamento local e os critérios de consistência, de convergência e de estabilidade.

\subsection{Aproximação de Derivadas por Diferenças Finitas}

Nesta seção desejamos mostrar o uso dos operadores diferenças finitas na aproximação das derivadas de uma função num ponto. Vamos primeiramente considerar uma função de uma variável $w(x)$ contínua, com derivadas contínuas. Através da fórmula de Taylor temos:

$$
\begin{aligned}
& w(x+h)=w(x)+h \frac{d w(x)}{d x}+\frac{h^{2}}{2} \frac{d^{2} w(x)}{d x^{2}}+\frac{h^{3}}{6} \frac{d^{3} w(x)}{d x^{3}}+\mathcal{O}\left(h^{4}\right) . \\
& w(x-h)=w(x)-h \frac{d w(x)}{d x}+\frac{h^{2}}{2} \frac{d^{2} w(x)}{d x^{2}}-\frac{h^{3}}{6} \frac{d^{3} w(x)}{d x^{3}}+\mathcal{O}\left(h^{4}\right) .
\end{aligned}
$$

Subtraindo a equação (2.2) de (2.1) obtemos uma aproximação para 
$d w / d x$ com erro de $\mathcal{O}\left(h^{2}\right)$ dada por:

$$
\frac{d w(x)}{d x}=\frac{1}{2 h}\{w(x+h)-w(x-h)\}+\mathcal{O}\left(h^{2}\right) .
$$

Também podemos obter outras aproximações para $d w / d x$ provenientes do truncamento das equações (2.1) e (2.2) no segundo termo, sendo estas:

$$
\begin{aligned}
& \frac{d w(x)}{d x}=\frac{1}{h}\{w(x+h)-w(x)\}+\mathcal{O}(h) . \\
& \frac{d w(x)}{d x}=\frac{1}{h}\{w(x)-w(x-h)\}+\mathcal{O}(h) .
\end{aligned}
$$

Introduzindo a notação de operadores diferenças ( Spiegel [21]), temos que as equações (2.3), (2.4) e (2.5) são respectivamente denotadas por:

$$
\frac{d w(x)}{d x} \simeq \frac{\delta w(x)}{2 h}
$$

onde $\delta w(x)=w(x+h)-w(x-h)$ é chamado de operador diferença central;

$$
\frac{d w(x)}{d x} \simeq \frac{\Delta w(x)}{h}
$$

onde $\Delta w(x)=w(x+h)-w(x)$ é chamado de operador diferença progressiva;

$$
\frac{d w(x)}{d x} \simeq \frac{\nabla w(x)}{h}
$$

onde $\nabla w(x)=w(x)-w(x-h)$ é chamado de operador diferença regressiva.

Geometricamente temos a seguinte situação (ver Figura 2.1): a fórmula (2.6) aproxima $d w / d x$ pela inclinação da corda $\overline{A B}$; a fórmula (2.7) aproxima $d w / d x$ pela inclinação da corda $\overline{P B}$; a fórmula (2.8) aproxima $d w / d x$ pela inclinação da corda $\overline{A P}$.

Para obtermos uma aproximação para $d^{2} w / d x^{2}$, somamos (2.1) com (2.2) resultando em:

$$
\frac{d^{2} w(x)}{d x^{2}}=\frac{1}{h^{2}}\{w(x+h)-2 w(x)+w(x-h)\}+\mathcal{O}\left(h^{2}\right) .
$$

Em certas ocasiões é conveniente aplicarmos o operador diferença central com passo $h / 2, \operatorname{logo} \delta w(x)=w(x+h / 2)-w(x-h / 2)$. Assim temos que $\delta^{2} w(x)=$ 


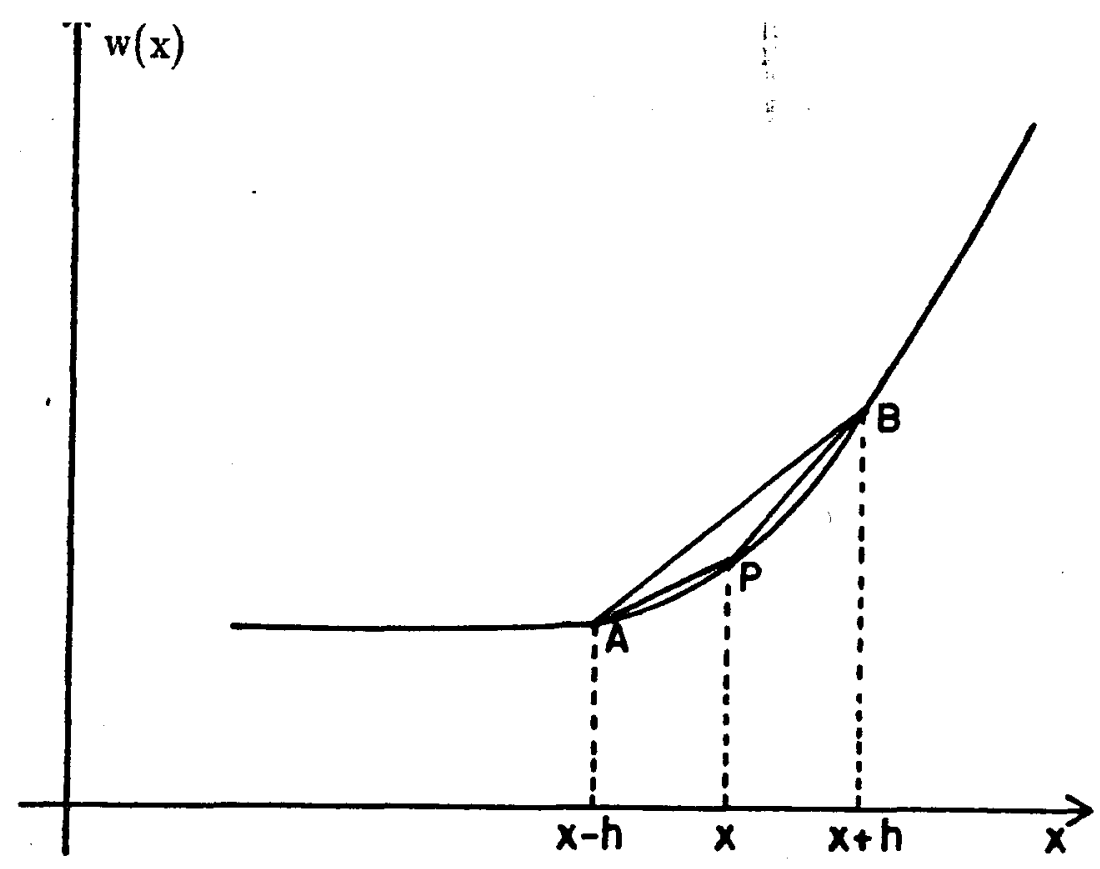

Figura 2.1:

$\delta(\delta w(x))=w(x+h)-2 w(x)+w(x-h)$ e conseqüentemente a equação (2.9) pode ser escrita da seguinte forma:

$$
\frac{d^{2} w(x)}{d x^{2}}=\frac{\delta^{2} w(x)}{h^{2}}+\mathcal{O}\left(h^{2}\right) .
$$

Consideremos agora funções de duas variáveis $p(x, t)$, com derivadas contínuas. Subdividindo o plano $(x, t)$ em um conjunto de retângulos de lados $h$ e $k$ (Figura 2.2), obtemos uma malha de pontos $(j k, n h)$. Denotando o valor da função $p(x, t)$ no ponto $(j k, n h)$ por $p_{j}^{n}$, obtemos através da fórmula de Taylor aproximaçôes para as derivadas parciais de $p(x, t)$ calculada em um ponto $(j k, n h)$, da seguinte forma:

$$
\begin{aligned}
&\left(p_{t}\right)_{j}^{n} \simeq \frac{\Delta_{t} p_{j}^{n}}{h}=\frac{p_{j}^{n+1}-p_{j}^{n}}{h} \\
&\left(p_{x}\right)_{j}^{n} \simeq \frac{\nabla_{x} p_{j}^{n}}{k}=\frac{p_{j}^{n}-p_{j-1}^{n}}{k} \\
&\left(p_{x}\right)_{j}^{n} \simeq \frac{\delta_{x} p_{j}^{n}}{2 k}=\frac{p_{j+1}^{n}-p_{j-1}^{n}}{2 k} \\
&\left(p_{x x}\right)_{j}^{n} \simeq \frac{\delta_{x}^{2} p_{j}^{n}}{k^{2}}=\frac{p_{j-1}^{n}-2 p_{j}^{n}+p_{j+1}^{n}}{k^{2}}
\end{aligned}
$$

Os índices $x$ e $t$ nos operadores, indicam em que variável estão sendo aplicados. 


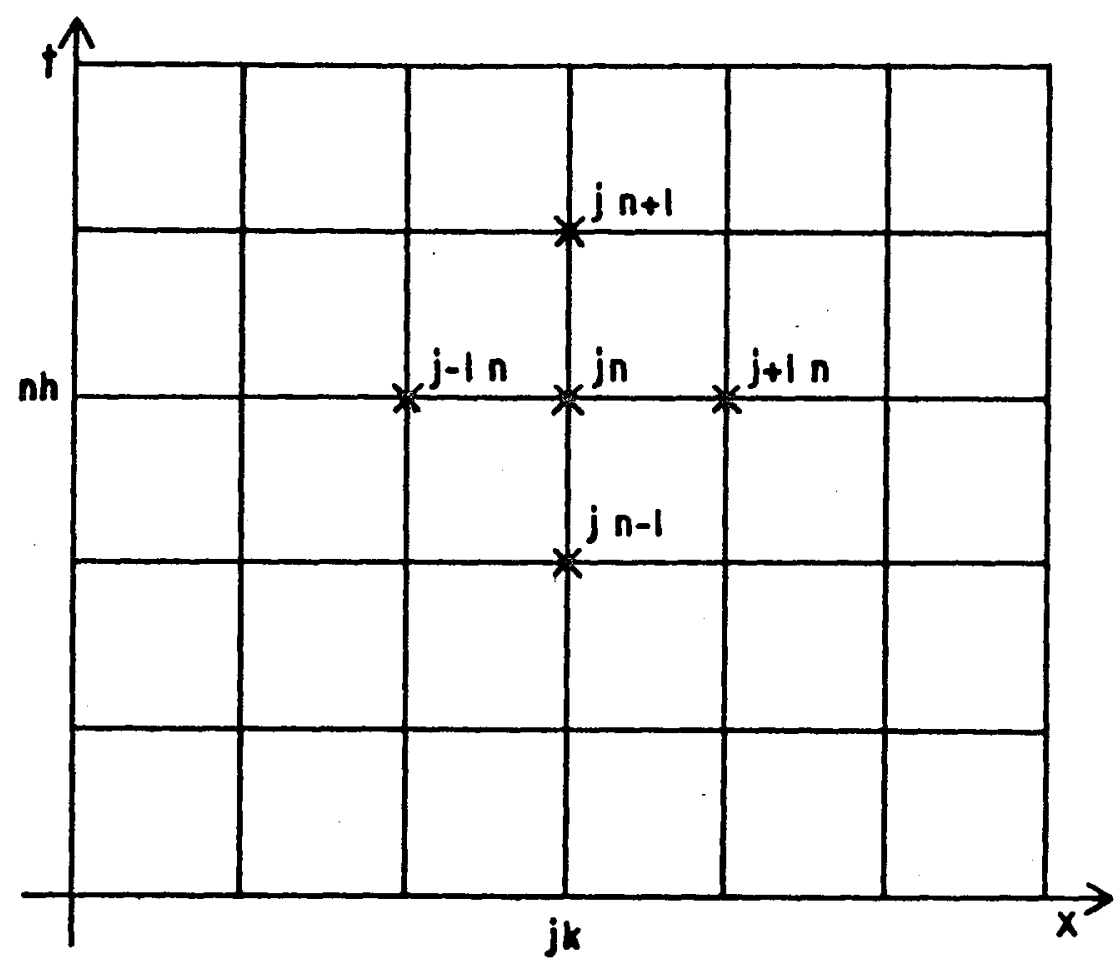

Figura 2.2:

Pode-se obter uma melhor aproximação para as derivadas usando a extrapolação para o limite,(Henrici [7]). Para mostrar este processo vamos considerar o caso de uma única variável. Subtraindo (2.2) de (2.1) obtemos a equação:

$$
w(x+h)-w(x-h)=2 h \frac{d w(x)}{d x}+\frac{h^{3}}{3} \frac{d^{3} w(x)}{d x^{3}}(x)+\mathcal{O}\left(h^{5}\right)
$$

Substituindo h por h/2 na equação acima temos:

$$
w(x+h / 2)-w(x-h / 2)=h \frac{d w(x)}{d x}+\frac{h^{3}}{24} \frac{d^{3} w(x)}{d x^{3}}(x)+\mathcal{O}\left(h^{5}\right) .
$$

Assim podemos eliminar o termo de $\mathcal{O}\left(h^{3}\right)$ subtraindo oito vezes (2.15) de (2.14) obtendo:

$$
\frac{d w(x)}{d x}=\frac{1}{6 h}\{w(x-h)+8[w(x+h / 2)-w(x-h / 2)]-w(x+h)\}+\mathcal{O}\left(h^{4}\right) .
$$

A desvantagem deste procedimento é o aumento do número de termos envolvidos na aproximação, acarretando um aumento no esforço computacional. 


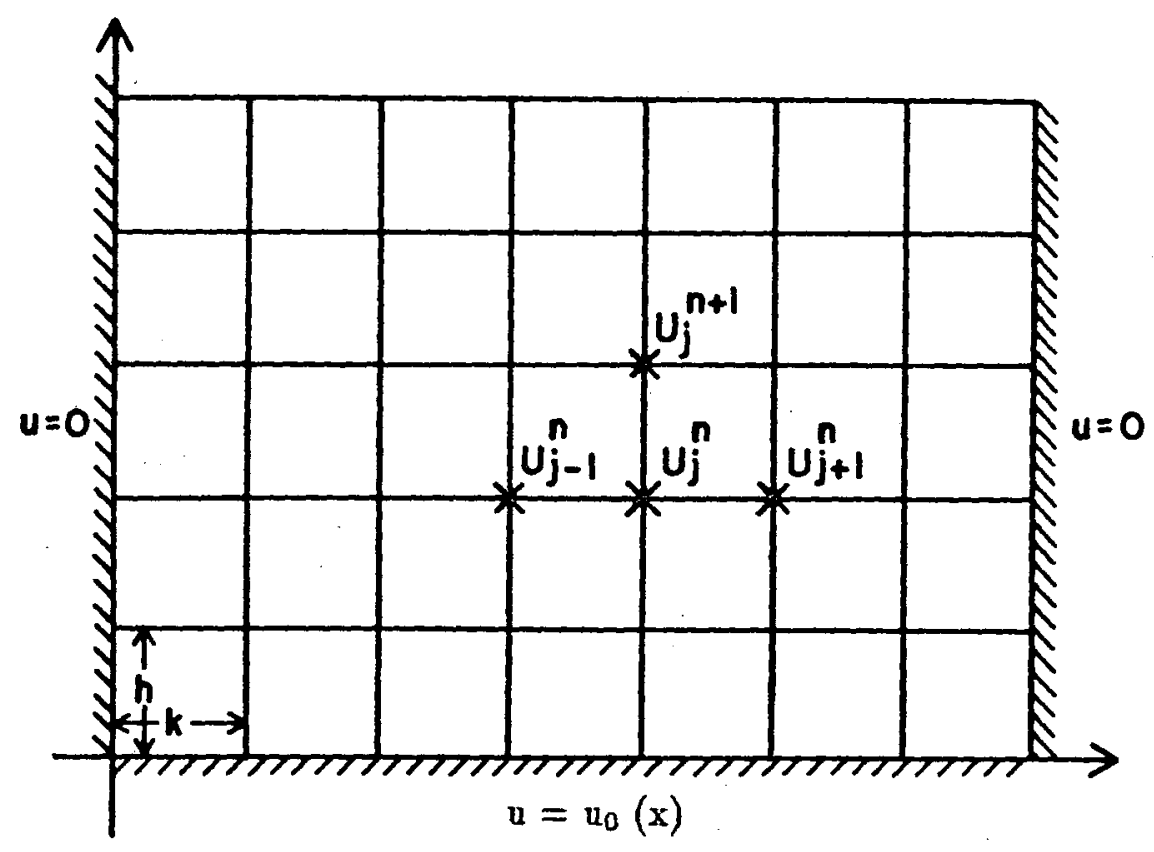

Figura 2.3: Esquema do método explícito

\subsection{Um Método Explícito}

Inicialmente vamos considerar o problema da condução do calor.

$$
\begin{cases}u_{t}(x, t)=u_{x x}(x, t) ; & x \in(0,1), t>0 \\ u(0, t)=u(1, t)=0 ; & t>0 \\ u(x, 0)=u_{0}(x) ; & x \in[0,1]\end{cases}
$$

Através dos operadores diferença (2.10) e (2.13) aproximamos o problema acima por:

$$
\left\{\begin{array}{l}
u_{j}^{n+1}=r u_{j+1}^{n}+(1-2 r) u_{j}^{n}+r u_{j-1}^{n} . \\
u_{0}^{n}=u_{j}^{n}=0 \\
u_{j}^{0}=u_{0}(j k) \quad j=1,2, \ldots, J-1
\end{array}\right.
$$

onde $r=h / k^{2}$ e $J k=1$.

Esta fórmula expressa um valor desconhecido da $(n+1)$-ésima linha de tempo em função dos valores conhecidos na $n$-ésima linha de tempo como mostra Figura 2.3. Assim, podemos aproximar a solução ao longo da primeira linha de tempo $t=h$ em termos dos valores iniciais $t=0$ e dos valores de fronteira, que são 
dados. Em seguida calculamos as aproximações na segunda, terceira,...,n-ésima linhas de tempo.

A este tipo de rnétodo, onde o cálculo de um valor desconhecido é função dos valores conhecidos, chamamos método explícito. Um método explícito é computacionalmente simples, mas tem sérias desvantagens. $O$ passo no tempo $h$ deve ser necessariamente muito pequeno, porque o processo só é estável quando $0 \leq r \leq 1 / 2$, como verernos no estudo da estabilidade.

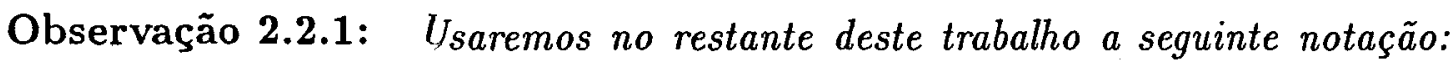
$u_{j}^{n}$ denota sempre a solução da equação diferença gerada pela discretização do problema contínuo; $U_{j}^{n}$ denotara a solução exata da EDP calculada no ponto $(j k, n h)$, isto é $U_{j}^{n}=u(j k, n h)$.

\subsection{Métodos de Crank-Nicolson}

Crank e Nicolson propuseram em 1.947 um novo método, que conjuga boas propriedades numb́ricas com um volume de cálculo reduzido. Eles aproximaram a equação $(2.17)$ no ponto $(j k,(n+1 / 2) h)$ da seguinte forma:

$$
\frac{1}{h} \delta_{t} u_{j}^{n+1 / 2}=\frac{1}{2 k^{2}} \delta_{x}^{2}\left(u_{j}^{n+1}+u_{j}^{n}\right)
$$

que nos fornecem a seguinte equação discreta:

$$
-r u_{j-1}^{n+1}+(2+2 \gamma) u_{j}^{n+1}-r u_{j+1}^{n+1}=r u_{j-1}^{n}+(2-2 r) u_{j}^{n}+r u_{j+1}^{n}
$$

Se a malha possuir $(J-1)$-pontos interiores ao longo de cada linha de tempo, (2.19) nos fornece um sistema linear tridiagonal com $(J-1)$-equações e $(J-1)$-incógnitas. Um método onde o cálculo de um valor desconhecido requer a solução de um sistema de equações é chamado de método implícito.

Generalizando o procedimento que Crank e Nicolson desenvolveram, obtemos uma classe geral de métodos para (2.17) dados por:

$$
\left\{\begin{array}{l}
u_{j}^{n+1}-u_{j}^{n}=r \delta_{x}{ }^{2}\left[\theta u_{j}^{n+1}+(1-\theta) u_{j}^{n}\right] \\
u_{0}^{n}=u_{J}^{n}=0 \\
u_{j}^{0}=u_{0}(j k) \quad j=1,2, \ldots, J-1
\end{array}\right.
$$

onde $0 \leq \theta \leq 1, j k=1$ e $r=h / k^{2}$.

Outros métodos para aproximação da equação (2.17) podem ser obtidos usando as mais diversas combinações de operadores a diferenças finitas. Em Richtmyer [17] (pag 189) temos uma relação destes métodos com as condições de convergência e estabilidade. 


\subsection{Condições de Fronteira a Derivadas}

No problema da equação do calor (1.2) tínhamos a superfície da barra isolada termicamente, logo o fluxo de calor na direção normal $(\eta)$ à superfície é igual a zero $(\partial u / \partial \eta=0)$.

Vamos considerar agora o caso onde a superfície não esteja isolada termicamente. A taxa de transferência de calor por radiação, através da superfície numa temperatura $u$, é muitas vezes assumida ser proporcional a $(u-v)$, onde $v$ é a temperatura externa. Uma suposição fundamental da Teoria do Calor é que a proporção do fluxo através de qualquer superfície é igual a $-C \partial u / \partial \eta$ unidades de calor por unidade de tempo, com isto a correspondente condição de fronteira é dada por:

$$
-C \frac{\partial u}{\partial \eta}=P(u-v)
$$

onde $C$ é a constante de condutividade térmica do material e $P$ é o coeficiente de transferência de calor da superfície, logo:

$$
\frac{\partial u}{\partial \eta}=-p(u-v)
$$

onde $p=P / C$.

Consideremos uma haste fina isolada termicamente ao longo de seu comprimento e que irradia calor através do extremo $x=0$, a temperatura neste extremo é desconhecida em tempo $t$, isto requer uma equação extra dada por:

$$
-u_{x}(0, t)=-p(u-v)
$$

o sinal negativo na derivada indica que o fluxo é em direção contrária ao eixo $x$.

Usando o operador diferença (2.10) na condição de fronteira obtemos:

$$
\frac{u_{1}^{n}-u_{0}^{n}}{k}=p\left(u_{0}^{n}-v\right) \text {. }
$$

Uma outra aproximação mais precisa é obtida usando o operador diferença central. Para isto seria necessário introduzimos uma temperatura ficticia $u_{-1}^{n}$ no ponto externo da malha $(-k, n h)$ ( supondo que a barra possa ser extendida Figura 2.4). Assim a condição de fronteira é representada por:

$$
\frac{u_{1}^{n}-u_{-1}^{n}}{2 k}=p\left(u_{0}^{n}-v\right)
$$




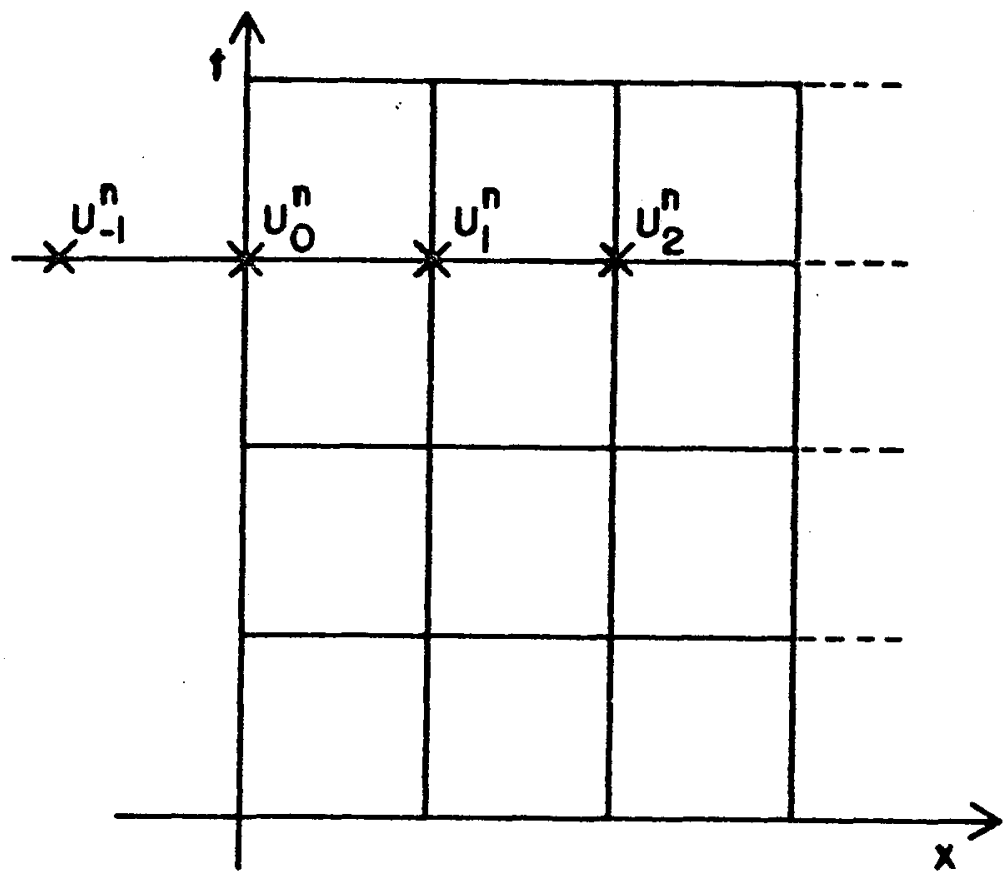

Figura 2.4:

Sendo $u_{-1}^{n}$ desconhecido necessitamos de outra equação. Assumindo que a equação do calor é satisfeita em $x=0$ e usando o método explícito para discretizá-la, obtem-se:

$$
u_{0}^{n+1}=u_{0}^{n}+r\left(u_{-1}^{n}-2 u_{0}^{n}+u_{1}^{n}\right)
$$

Eliminando $u_{-1}^{n}$ das equações (2.22) e (2.21) obtemos :

$$
u_{0}^{n+1}=u_{0}^{n}+r\left\{-2 k p\left(u_{0}^{n}-v\right)-2 u_{0}^{n}+2 u_{1}^{n}\right\} .
$$

Outras condições de contorno, que envolvam derivadas, podem ser tratadas de maneira análoga.

\subsection{Erro de Truncamento Local}

Como salientado na observação (2.2.1) denotamos por $u_{j}^{n}$ a solução exata da equação diferença e por $U_{j}^{n}$ o valor da solução exata da $\operatorname{EDP}$ no ponto $(j k, n h)$. Em geral os valores $U_{j}^{n}$ não satisfazem a equação diferença. Temos por conseguinte a definição: 
Definição 2.5.1: $O$ valor $T_{j}^{n}$, obtido da substituição de $U_{j}^{n}$ na equação diferença é chamado de erro de truncamento local no ponto $(j k, n h)$ da malha.

Vamos expressar $T_{j}^{n}$ de (2.20) em termos de potências de $h$ e $k$ e das derivadas parciais de $u$ em $(j k, n h)$, usando a fórmula de Taylor. Apesar de $u$ e suas derivadas não serem conhecidas de um modo geral, esta análise é conveniente, porque ela produz um método de comparação da precisão local de diferentes métodos. Assim pela definição de $T_{j}^{n}$ temos:

$$
\begin{aligned}
T_{j}^{n}= & -r \theta U_{j-1}^{n+1}+(1+2 r \theta) U_{j}^{n+1}-r \theta U_{j+1}^{n+1}- \\
& (1-\theta) r U_{j-1}^{n}+[1+2 r(1-\theta)] U_{j}^{n}-(1-\theta) r U_{j+1}^{n},
\end{aligned}
$$

Expandindo a solução $u$ por Taylor no ponto $(j k, n h)$ obtemos:

$$
\begin{aligned}
U_{j+1}^{n} & =u\left(x_{j}+k, t_{n}\right)=U_{j}^{n}+k\left(u_{x}\right)_{j}^{n}+\frac{1}{2} k^{2}\left(u_{x x}\right)_{j}^{n}+\frac{1}{6} k^{3}\left(u_{x x x}\right)_{j}^{n}+\cdots \\
U_{j-1}^{n} & =u\left(x_{j}-k, t_{n}\right)=U_{j}^{n}-k\left(u_{x}\right)_{j}^{n}+\frac{1}{2} k^{2}\left(u_{x x}\right)_{j}^{n}-\frac{1}{6} k^{3}\left(u_{x x x}\right)_{j}^{n}+\cdots ; \\
U_{j}^{n+1} & =u\left(x_{j}, t_{n}+h\right)=U_{j}^{n}+h\left(u_{t}\right)_{j}^{n}+\frac{1}{2} h^{2}\left(u_{t t}\right)_{j}^{n}+\frac{1}{6} h^{3}\left(u_{t t t}\right)_{j}^{n}+\cdots \\
U_{j}^{n-1} & =u\left(x_{j}, t_{n}-h\right)=U_{j}^{n}-h\left(u_{t}\right)_{j}^{n}+\frac{1}{2} h^{2}\left(u_{t t}\right)_{j}^{n}-\frac{1}{6} h^{3}\left(u_{t t t}\right)_{j}^{n}+\cdots
\end{aligned}
$$

e substituindo na expressão (2.23) obtemos:

$$
\begin{aligned}
T_{j}^{n}= & \left(u_{t}-u_{x x}\right)_{j}^{n}+h\left[\left(\frac{1}{2} u_{t}-\theta u_{x x}\right)_{t}\right]_{j}^{n}+\frac{h^{2}}{2}\left(\frac{1}{3} u_{t t t}-\theta u_{x x t}\right)_{j}^{n}- \\
& \frac{k^{2}}{12}\left(u_{x x x x}\right)_{j}^{n}+\cdots
\end{aligned}
$$

Logo sendo $u$ solução da EDP, então $T_{j}^{n}=\mathcal{O}(h)+\mathcal{O}\left(k^{2}\right)$. Quando $\theta=1 / 2$ temos que o termo de $\mathcal{O}(h)$ se anula, logo o método de Crank-Nicolson tem erro de truncamento de ordem $\mathcal{O}\left(h^{2}\right)+\mathcal{O}\left(k^{2}\right)$.

\subsection{Consistência ou Compatibilidade}

Pode ocorrer que a aproximação numérica de uma EDP convirja para a solução de outra EDP, quando o tamanho da malha tende a zero. Quando isto ocorre dizemos que o método de diferenças é inconsistente ou incompatível. Definimos consistência em função do erro de truncamento local, como: 
Definição 2.6.1: Uma equação diferença é consistente se o valor limite do erro de truncamento local é zero quando a malha tende a zero.

Por (2.24) temos que $T_{j}^{n} \rightarrow 0$ quando $h, k \rightarrow 0$, logo os métodos de Crank-Nicolson são consistentes.

\subsection{Convergência}

O problema de convergência é posto como a determinação de condições suficientes para que a solução exata da equação diferença convirja para a solução exata da EDP, quando diminuimos o tamanho da malha, isto é:

Definição 2.7.1: Sejam $x_{j}=j k$ e $t_{n}=n h . A$ equação diferença é dita ser convergente no ponto $\left(x_{j}, t_{n}\right)$ se $u_{j}^{n} \rightarrow U_{j}^{n}$, quando $h \rightarrow 0$ e $k \rightarrow 0$. A equação é dita ser convergente se esta é convergente para todos os pontos do domínio.

As condições de convergência, aqui estabelecidas, são para equações parabólicas lineares de segunda ordem. Para equações não lineares não existe uma teoria geral, aplicando-se um tratamento caso a caso.

A diferença $U_{j}^{n}-u_{j}^{n}$ é chamada de erro de discretização. Alguns autores chamam esta diferença de erro de truncamento, mas deixaremos este termo para a diferença entre a equação diferencial e sua aproximação por diferenças finitas. A magnitude do erro de discretização em qualquer ponto da malha depende do tamanho da malha $k$ e $h$. O erro de discretização deve (teoricamente) diminuir com o decréscimo de $k$ e $h$, porém o número de equações a serem resolvidas aumenta na mesma proporção. Portanto existe uma limitação devido ao custo computacional.

Em geral o problema da convergência é um problema difícil de ser investigado convenientemente, pois a expressão final do erro de discretização envolve derivadas desconhecidas para as quais nem sempre pode ser calculado um limitante. Lax e Richtmyer relacionaram convergência com estabilidade via o Teorema de Equivalência de Lax, onde a estabilidade é definida como:

Definição 2.7.2: A equação diferença é dita ser estável se o erro de discretização permanece limitado quando a malha tende a zero numa região limitada. 
Teorema 2.7.1 (Equivalência de Lax): Considere um problema de valor inicial e sua aproximação por diferenças finitas. Esta aproximação será convergente se, e somente se, ela for estável e consistente.

Prova: ver Richtmyer [17].

Da definição de $U_{j}^{n}$ e $u_{j}^{n}$ temos que o erro em cada ponto da malha é dado por $e_{j}^{n}=U_{j}^{n}-u_{j}^{n}$ e portanto $u_{j}^{n}=U_{j}^{n}-e_{j}^{n}$. Assim a equação para o erro no caso do método explicito (2.18) fica:

$$
e_{j}^{n+1}=r e_{j-1}^{n}+(1-2 r) e_{j}^{n}+r e_{j+1}^{n}-r\left(U_{j-1}^{n}-2 U_{j}^{n}+U_{j+1}^{n}\right)-U_{j}^{n}+U_{j}^{n+1} .
$$

Através da fórmula de Taylor temos:

$$
\begin{aligned}
U_{j+1}^{n} & =u\{(j+1) k, n h\}=U_{j}^{n}+k u_{x}(j k, n h)+\frac{k^{2}}{2} u_{x x}\left(x_{j}+\theta_{1} k, t_{n}\right) \\
U_{j-1}^{n} & =u\{(j-1) k, n h\}=U_{j}^{n}-k u_{x}(j k, n h)+\frac{k^{2}}{2} u_{x x}\left(x_{j}-\theta_{2} k, t_{n}\right) \\
U_{j}^{n+1} & =u\{j k,(n+1) h\}=U_{j}^{n}+h u_{t}\left(x_{j}, t_{n}+\theta_{3} h\right)
\end{aligned}
$$

onde $0 \leq \theta_{i} \leq 1$ para $i=1,2,3$ e substituindo na equação temos:

$$
e_{j}^{n+1}=r e_{j-1}^{n}+(1-2 r) e_{j}^{n}+r e_{j+1}^{n}+h T(j k, n h),
$$

onde

$$
T(j k, n h)=\left\{u_{t}\left(x_{j}, t_{n}+\theta_{3} h\right)-u_{x x}\left(x_{j}+\theta_{4} k, t_{n}\right)\right\} \quad \text { com }-1<\theta_{4}<1 .
$$

Seja $E_{n}=\max \left|e_{j}^{n}\right|$ ao longo da $n$-ésima linha de tempo e seja $M=\max |T(j k, n h)|$, para todo $j, n$. Quando $0<r \leq 1 / 2$ todos os coeficientes de $e$ na equação (2.26) são não negativos, logo:

$$
\begin{aligned}
\left|e_{j}^{n+1}\right| & \leq r\left|e_{j-1}^{n}\right|+(1-2 r)\left|e_{j}^{n}\right|+r\left|e_{j+1}^{n}\right|+h M \\
& \leq r E_{n}+(1-2 r) E_{n}+r E_{n}+h M \\
& =E_{n}+h M .
\end{aligned}
$$

Sendo a expressão verdadeira para todos valores de $e_{j} j=0,1, \ldots, J$, também é verdadeira para $\max _{j}\left|e_{j}^{n+1}\right|$, conseqüentemente temos:

$$
E_{n+1} \leq E_{n}+h M \leq\left(E_{n-1}+h M\right)+h M \leq \ldots \leq E_{0}+(n+1) h M .
$$

Como os valores iniciais $u_{j}^{n}$ e $U_{j}^{n}$ são iguais, então $E_{0}=0$, portanto temos:

$$
E_{n} \leq n h M=t M \text {. }
$$


Quando $k \rightarrow 0$ temos que $h=r k^{2} \rightarrow 0$, portanto:

$$
M \rightarrow\left|\left(u_{t}-u_{x x}\right)_{j}^{n}\right|=0 \Rightarrow E_{n} \rightarrow 0,
$$

e portanto o método é convergente.

Desta forma temos que uma condição suficiente para que o método explícito seja convergente é que $r \leq 1 / 2$.

Consideremos $r>1 / 2$ e vamos analisar o que ocorre com o erro de discretização. Na equação (2.26) temos uma equação diferença não homogênea, com coeficientes constantes, cuja solução geral é igual a uma solução particular mais a solução geral da equação homogênea, assim consideremos o problema:

$$
\phi_{j}^{n+1}=r \phi_{j-1}^{n}+(1-2 r) \phi_{j}^{n}+r \phi_{j+1}^{n} \text {. }
$$

Vamos procurar uma solução da forma $\phi_{j}^{n}=e^{\lambda n} e^{i \beta j}$, onde $\beta$ é real. Substituindo na equação acima obtemos:

$$
e^{\lambda} \phi_{j}^{n}=(1-2 r) \phi_{j}^{n}+\phi_{j}^{n} r\left[e^{-\imath \beta}+e^{\imath \beta}\right] .
$$

Assim para que $\phi_{j}^{n}$ seja solução, é necessário que:

$$
e^{\lambda}=1-2 r(1-\cos (\beta))=1-4 r \operatorname{sen}^{2}\left(\frac{\beta}{2}\right) \text {. }
$$

Sendo $r>1 / 2$ temos que $\left|e^{\lambda}\right|>1, \operatorname{logo} \phi_{j}^{n}$ é ilimitado quando $n \rightarrow \infty$, logo existem soluções $e_{j}^{n}$ que crescem exponencialmente quando $n \rightarrow \infty$. Assim $r \leq 1 / 2$ é condição necessária para convergência

\subsection{Estabilidade Absoluta}

A idéia essencial atrás do conceito de estabilidade é que o processo numérico, à medida que evolui, deveria limitar os erros cometidos nos passos anteriores e não propagá-los. O conceito de estabilidade apresentado por Lax e Richtmyer (Definição 2.7.2) não é de aplicação prática, pois não utiliza-se um método numérico com a malha tendendo a zero. Os passos $h$ e $k$ são normalmente mantidos constantes quando a solução é propagada no tempo, e muitos autores definem estabilidade em termos da limitação da solução numérica para malha de tamanho fixo, ou seja:

Definição 2.8.1: A equação diferença é dita ser absolutamente estável para uma malha de tamanho fixo, se o erro de discretização, quando $n \rightarrow \infty$, permanece limitado. 
Este conceito será adotado daqui em diante. Em particular fixaremos o valor de $k$ (pequeno), conseqüentemente $J$ será fixo. Estudaremos a seguir critérios para caracterização da estabilidade para (2.20).

Supondo que a condição inicial $u_{o}(x)$ possa ser expandida numa série de senos, mostraremos a seguir que a solução de (2.20) também tem essa propriedade. De fato, sejam $A, \xi$ e $s$ constantes e $s$ inteiro. Substituindo na equação (2.20) a expressão:

$$
u_{j}^{n}=A \xi^{n} \operatorname{sen}\left(\frac{s \pi j}{J}\right)
$$

temos que esta será satisfeita se:

$$
\xi=\frac{1-4 r(1-\theta) \operatorname{sen}^{2}\left(\frac{s \pi}{2 J}\right)}{1+4 r \theta \operatorname{sen}^{2}\left(\frac{s \pi}{2 J}\right)} .
$$

Desta forma $u_{j}^{n}$ definido por:

$$
u_{j}^{n}=\sum_{s=-\infty}^{+\infty} A_{s} \xi_{s}{ }^{n} \operatorname{sen}\left(\frac{s \pi j}{J}\right)
$$

onde os $A_{s}$ são os coeficientes de Fourier da condição inicial $u_{0}$, é a solução exata de (2.20), pois:

a) A série (2.28) é absolutamente convergente, dado que a série para $u_{o}(x)$ é absolutamente convergente e os $\xi_{s}, s=0,1, \ldots, J$ são limitados em $s$.

b) Cada termo da série (2.28) satisfaz a equação diferença e portanto a soma também.

c) Para $n=0(2.28)$ se reduz a série de $u_{0}(j k)$ e desta forma satisfaz a condição inicial.

d) Para $j=0$ e $j=J$ os termos da série se anulam, logo a série satisfaz as condições de fronteira.

Comparando o fator de crescimento do problema contínuo (ver (1.6)) com o do problema discreto, podemos obter os critérios de estabilidade em função de $\xi_{s}$, como vemos no teorema que segue.

Teorema 2.8.1 (Critério de Estabilidade Absoluta): Suponha que a série de Fourier para $u_{0}(x)$ seja absolutamente convergente. Então o erro de discretização $U_{j}^{n}-u_{j}^{n}$ permanece limitado quando $n \rightarrow \infty$, para $k$ e fixos se, e somente se:

$$
\max _{s}\left|\xi_{s}\right| \leq 1
$$




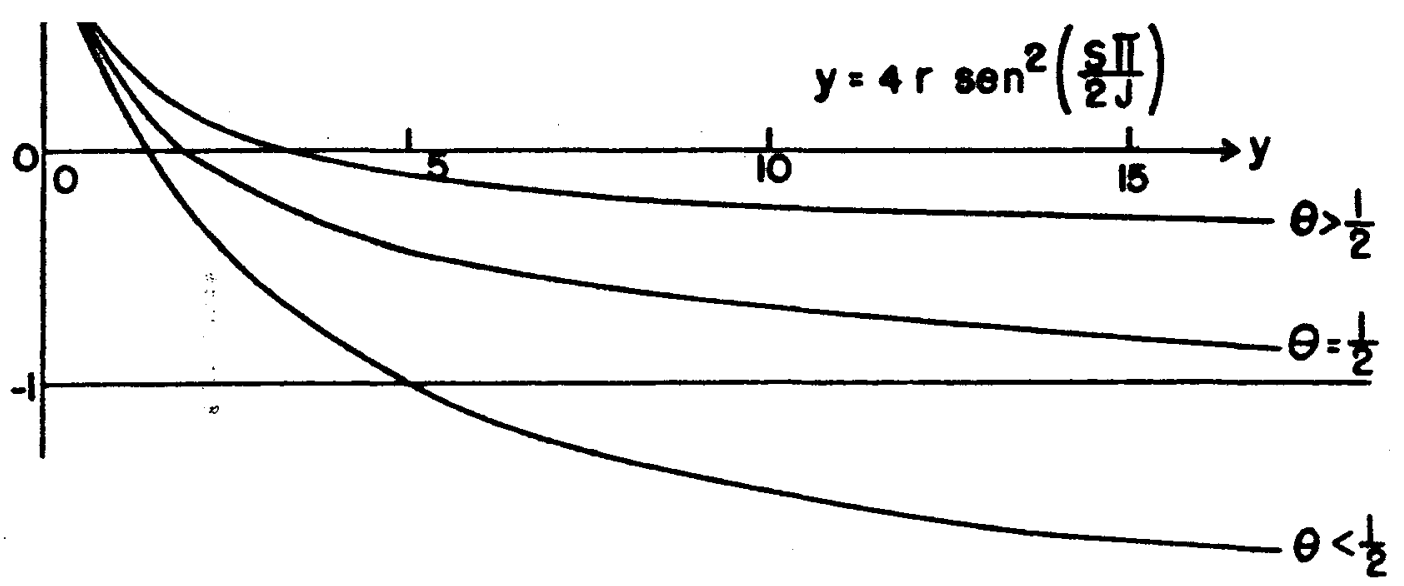

Figura 2.5: Fator de crescimento para métodos de Crank-Nicolson.

Prova: Desde que u(x,t) é a solução exata do problema contínuo, esta é limitada quando $t \rightarrow \infty$. Então o erro será limitado se, e somente se, $u_{j}^{n}$ for limitado quando $n \rightarrow \infty$, porém:

$$
\begin{aligned}
\left|u_{j}^{n}\right| & =\left|\sum_{s=-\infty}^{+\infty} A_{s} \xi_{s}^{n} \exp \left(\frac{i s \pi j}{J}\right)\right| \\
& \leq \sum_{s=-\infty}^{+\infty}\left|A_{s} \| \xi_{s}\right|^{n} \\
& \leq \sum_{s=-\infty}^{+\infty}\left|A_{s}\right| .
\end{aligned}
$$

Desde que a série de Fourier de $u_{0}$ é absolutamente convergente, então $u_{j}^{n}$ é limitado.

Por outro lado se $\left|\xi_{s}\right|>1$ para algum $s=s_{1}$, é suficiente que tomemos $u_{0}(x)=\operatorname{sen}\left(s_{1} \pi x\right)$ para exibir uma solução ilimitada em $n$ dada por:

$$
u_{j}^{n}=\xi_{s_{1}}^{n} \operatorname{sen}\left(\frac{s_{1} \pi j}{J}\right)
$$

o que prova o teorema.

Na Figura (2.5) mostramos os valores de $\xi_{s}$ como uma função de $y=4 r \operatorname{sen}^{2}(s \pi / 2 J)$, para vários valores de $\theta$. Quando $y$ cresce através de 


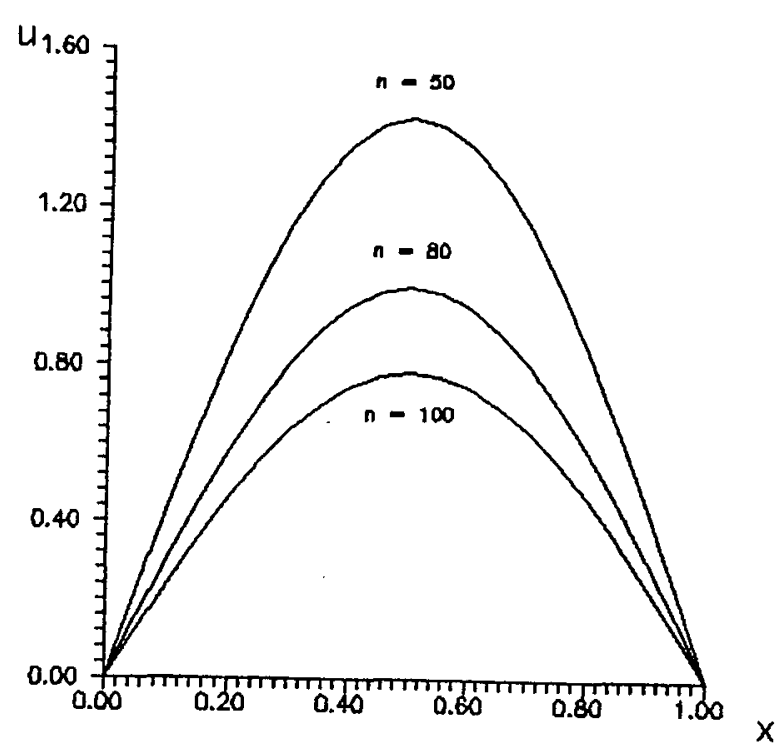

Figura 2.6: Solução da equação diferença com $h=0.0012$

valores positivos, os valores de $\xi_{s}$ decrescem monotonicamente de 1 para $-(1-$ $\theta) / \theta$. Se $0.5 \leq \theta \leq 1$ o comportamento assintótico de $\xi_{s}$ é maior que -1 . Se $0 \leq \theta<0.5$ o comportamento assintótico de $\xi_{s}$ é menor que -1 . Logo $y$ deve ser restrito a valores para os quais a curva intercepta a linha $\xi_{s}=-1$. Com isto concluimos que as condições de estabilidade para os métodos de Crank-Nicolson são:

(i) $\operatorname{Se} \theta \in[0.5,1]$, então $r>0$;

(ii) $\operatorname{Se} \theta \in[0,0.5)$, então $r \leq \frac{1}{2(1-2 \theta)}$.

A Figura 2.6 mostra a solução de (2.20) $\operatorname{com} \theta=0, J=20, u_{0}(x)=$ $10\left(-x^{2}+x\right)$ e $h=0.0012$. Neste caso $r=0.48<0.5$, e portanto satisfaz o critério de estabilidade. A Figura 2.7 foi gerada com os mesmos parâmetros que a Figura 2.6 , exceto que $h=0.0013(r=0.52>0.5)$. Neste caso o critério de estabilidade não é satisfeito, e podemos ver a instabilidade começando a se manifestar com $n=80$. Conforme $n$ cresce a solução tem um crescimento ilimitado, contrariando assim o comportamento assintótico da solução analítica ( ver (1.6) ). 


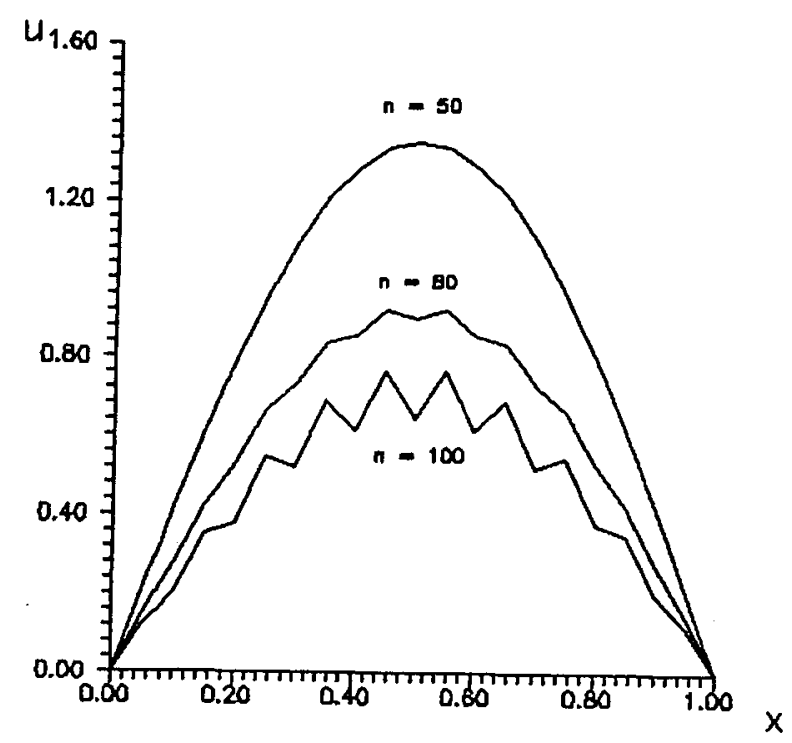

Figura 2.7: Solução da equação diferença com $h=0.0013$

\subsection{Equação Não Linear}

Até o momento consideramos uma equação parabólica linear, porém muitos problemas têm como modelo equações não lineares. Não existe dificuldade em aplicar formalmente os métodos de diferenças finitas para equações parabólicas nâo lineares. A forma de uma equação parabólica não linear de segunda ordem é:

$$
u_{t}=f\left(x, t, u, u_{x}, u_{x x}\right) .
$$

Um método explícito pode ser obtido de forma análoga ao caso linear, e uma aproximação para (2.29) é dada por:

$$
\frac{u_{j}^{n+1}-u_{j}^{n}}{h}=f\left(x_{j}, t_{n}, u_{j}^{n}, \frac{\delta_{x} u_{j}^{n}}{2 k}, \frac{\delta_{x}^{2} u_{j}^{n}}{k^{2}}\right) .
$$

Este método, ainda que produza uma equação fácil de se resolver, sofre do problema de estabilidade. No caso linear, como foi mostrado anteriormente, o passo $h$ é limitado por $h / k^{2} \leq 0.5$, já no caso não linear esta limitação certamente depende de $f$.

Considerando os métodos de Crank-Nicolson, a equação (2.29) pode ser 
aproximada por:

$$
\frac{u_{j}^{n+1}-u_{j}^{n}}{h}=f\left(x_{j}, t_{n}, w_{n}, \frac{\delta_{x} w_{n}}{2 k}, \frac{\delta_{x}{ }^{2} w_{n}}{k^{2}}\right),
$$

onde $w_{n}=\theta u_{j}^{n+1}+(1-\theta) u_{j}^{n}$ e $0 \leq \theta \leq 1$.

Infelizmente o problema algébrico a ser resolvido é mais complicado, pois (2.30) representa um sistema de equações não lineares (quando $\theta \neq 0$ ). Em geral métodos diretos são difíceis de serem aplicados neste caso. Assim, estas equações são resolvidas iterativamente após serem linearizadas por algum processo. Apresentamos aqui dois métodos, o método de Newton e o método de Três Níveis de Lees.

Inicialmente analisaremos o método de Newton, para isto vamos considerar um sistema não linear com $N$-equações e $N$-incógnitas $u_{1}, u_{2}, \ldots, u_{N}$ dado por:

$$
f_{i}\left(u_{1}, u_{2}, \ldots, u_{N}\right)=0 ; \quad i=1,2, \ldots, N
$$

Seja $v_{i}$ uma aproximação conhecida para a solução exata $u_{i}, \operatorname{com} i=$ $1,2, \ldots, N$. Fazendo $u_{i}=v_{i}+\varepsilon_{i}$, substituindo na equação e usando a expansão de Taylor em $\varepsilon_{i}$ obtemos:

$$
f_{i}\left(v_{1}, v_{2}, \ldots, v_{N}\right)+\left[\left(f_{i}\right)_{u_{1}}\left(v_{1}\right) \varepsilon_{1}+\cdots+\left(f_{i}\right)_{u_{N}}\left(v_{N}\right) \varepsilon_{N}\right]=0
$$

A equação (2.31) representa um sistema linear de $N$-equações para $N$-incógnitas $\varepsilon_{1}, \ldots, \varepsilon_{N}$. Após o cálculo dos $\varepsilon$ 's o processo se repete, onde a nova aproximação é dada por $v_{i}+\varepsilon_{i}$, até que os $u_{i}$ 's tenham sido encontrados com o grau de precisão desejado.

Lees [12] considerou a equação não linear da forma:

$$
b(u) u_{t}=\left(a(u) u_{x}\right)_{x} ; a(u)>0, \quad b(u)>0
$$

e investigou um método que:

(i ) realiza a linearização nos $u_{j}^{n+1}$ através do cálculo de todos os coeficientes de $u_{j}^{n+1}$ em uma linha de tempo conhecida.

(ii) preserva a estabilidade através do cálculo de $u_{j}^{n}$ em três níveis de tempo.

(iii) conserva precisão através do uso do operador diferença central. 
Usando a aproximação por operador diferença central em (2.32) obtemos:

$$
b\left(u_{j}^{n}\right) \frac{1}{2 h}\left(u_{j}^{n+1}-u_{j}^{n-1}\right)=\frac{1}{k^{2}}\left\{a\left(u_{j+1 / 2}^{n}\right)\left(u_{j+1}^{n}-u_{j}^{n}\right)-a\left(u_{j-1 / 2}^{n}\right)\left(u_{j}^{n}-u_{j-1}^{n}\right)\right\}
$$

que é instável para $a \equiv b \equiv 1$.

Para evitar os pontos médios $j \pm 1 / 2$, Lees substituiu $a\left(u_{j+1 / 2}^{n}\right)$ e $a\left(u_{j-1 / 2}^{n}\right)$ por $a\left\{1 / 2\left(u_{j+1}^{n}+u_{j}^{n}\right)\right\}$ e $a\left\{1 / 2\left(u_{j}^{n}+u_{j-1}^{n}\right)\right\}$ respectivamente, e também substituiu $u_{j+1}^{n}, u_{j}^{n}$ e $u_{j-1}^{n}$ por:

$$
\begin{gathered}
\frac{1}{3}\left(u_{j+1}^{n+1}+u_{j+1}^{n}+u_{j+1}^{n-1}\right) \\
\frac{1}{3}\left(u_{j}^{n+1}+u_{j}^{n}+u_{j}^{n-1}\right) \\
\frac{1}{3}\left(u_{j-1}^{n+1}+u_{j-1}^{n}+u_{j-1}^{n-1}\right)
\end{gathered}
$$

respectivamente. Com isto Lees provou que para $h$ e $k$ suficientemente pequeno existe $A$ constante tal que:

$$
\max _{j n}\left|U_{j}^{n}-u_{j}^{n}\right| \leq A\left(h^{2}+k^{2}\right)
$$

Com a substituição acima vemos que os valores na $(n+1)$-ésima linha de tempo dependem dos valores nas $n$ e $(n-1)$-ésima linhas (Três Niveis). Logo necessitamos dos valores nas duas primeiras linhas de tempo para avançar um passo. A primeira linha $u_{j}^{0}$ é dada pela condição inicial. A segunda linha $u_{j}^{1}$ é obtida através da equação diferença considerando $u_{j}^{-1} \equiv 0$, por exemplo. 


\section{Capítulo 3}

\section{Equação Reação-Difusão}

Neste capítulo estudamos a equação parabólica conhecida como Equação Reação-Difusão. Este problema é encontrado no modelamento de muitos fenômenos químicos e biológicos.

Iniciamos com uma introdução à Teoria da Bifurcação e analisamos o problema através de elementos desta teoria, estabilidade linear e estabilidade não linear fraca.

\subsection{Introdução à Teoria da Bifurcação} da forma:

A Teoria da Bifurcação é o estudo de soluções de equilíbrio em equações

$$
w_{t}=G(\lambda, w),
$$

onde $\lambda$ é um parâmetro e $G: \Lambda \times H \rightarrow H$ é um operador sobre os espaços de Banach $\Lambda$ e $H$. As soluções mais simples que (3.1) pode ter são soluções de equilíbrio ( também chamadas de soluções estáticas ), isto é, independentes do tempo. Logo estas soluções devem satisfazer:

$$
G(\lambda, w)=0 .
$$

Certas propriedades dos problemas não lineares são determinadas através do problema linerizado. Para isto nescessitamos do conceito de Frechet diferenciavel dado por:

Definição 3.1.1 (Frechet Diferenciavel): Seja $h: A \subseteq X \rightarrow Y$ onde $X$ e $Y$ são espaços de Banach. O operador $h$ é dito Frechet diferenciavel em $x_{0} \in A$, se 
existe $T: X \rightarrow Y$, operador linear contínuo, tal que:

$$
\lim _{x \rightarrow x_{0}} \frac{h(x)-h\left(x_{0}\right)-T\left(x-x_{0}\right)}{\left\|x-x_{0}\right\|}=0
$$

A principal ferramenta em Teoria da Bifurcação é o Teorema da Função Implícita dado por:

Teorema 3.1.1 (Função Implícita): Sejam $A \subseteq \Lambda \times H:$ um aberto $e G: A \rightarrow$ $H$ Frechet diferenciavel em $A$. Sejam também $\left(\lambda_{0}, w_{0}\right) \in A$ e $G\left(\lambda_{0}, w_{0}\right)=c$ e suponha que a derivada de Frechet de $G$, é contínua em $\left(\lambda_{0}, w_{0}\right)$ e que $G_{w}\left(\lambda_{0}, w_{0}\right)$ $\dot{e}$ um isomorfismo. Então existem os abertos $V \subset \Lambda$ contendo $\lambda_{0}$ e $Z \subset \Lambda \times H$ contendo $\left(\lambda_{0}, w_{0}\right)$ com as seguintes propriedades:

(i ) Existe uma função Frechet diferenciavel, $g: V \rightarrow H$ tal que $g\left(\lambda_{0}\right)=w_{0}$ e que para cada $\lambda \in V$ temos que $(\lambda, g(\lambda)) \in Z$ e $G(\lambda, g(\lambda))=c$

( ii) $g^{\prime}(\lambda)=-\left[G_{w}(\lambda, g(\lambda))\right]^{-1} \circ G_{\lambda}(\lambda, g(\lambda))$

Prova: (ver Flett [6]).

- À curva da equação $G(\lambda, w)=0$ denominamos de diagrama de bifurcação. Damos a seguir a classificação dos pontos sobre esta curva.

(1) Um ponto $\left(\lambda_{0}, w_{0}\right)$ é um ponto regular de $G(\lambda, w)=0$ se a ele podemos aplicar o teorema da Função Implícita ( Figura 3.1 (a)).

(2) Um ponto $\left(\lambda_{0}, w_{0}\right)$ não regular é dito ser singular, ou seja $G_{w}\left(\lambda_{0}, w_{0}\right)$ não é inversível (Figura 3.1 (b) ).

(3) Um ponto $\left(\lambda_{0}, w_{0}\right)$ é um ponto de bifurcação se ele for um ponto singular, e se em qualquer vizinhança de $\left(\lambda_{0}, w_{0}\right)$ existam $\left(\lambda^{*}, w_{1}\right)$ e $\left(\lambda^{*}, w_{2}\right)$ distintos tais que $\left(\lambda^{*}, w_{1}\right)$ e $\left(\lambda^{*}, w_{2}\right)$ satisfaçam $G(\lambda, w)=0$. Em outras palavras, por $\left(\lambda_{0}, w_{0}\right)$ passam no minimo dois ramos da curva $G(\lambda, w)=0$ (Figura 3.1 (c) ).

(4) Um ponto duplo é um ponto de bifurcação onde passam duas, e somente duas curvas que satisfaçam $G(\lambda, w)=0$. (Figura 3.1 (d) ).

(5) Um ponto $\left(\lambda_{0}, w_{0}\right)$ é um ponto duplo de retorno se ele é um ponto duplo no qual dois dos quatro ramos existentes tem tangente de sinais opostos na vizinhança do ponto ( Figura 3.1 (e) ). 


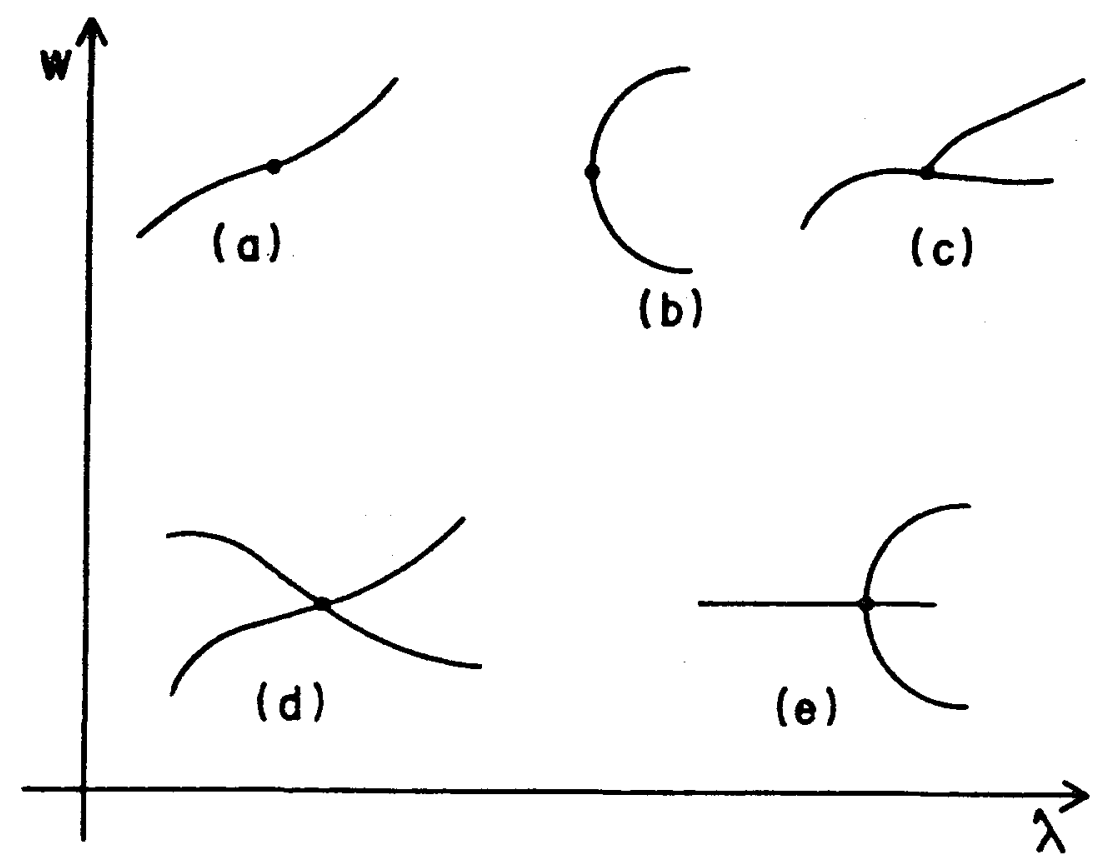

Figura 3.1: Classificação dos pontos de bifurcação: (a) Ponto regular; (b) Ponto singular; (c) Ponto de bifurcação; (d) Ponto duplo; (e) Ponto duplo de retorno.

Assumindo que existe uma curva conhecida $w=g(\lambda)$ das soluções de $G(\lambda, w)=0$, podemos fazer uma mudança de variável em (3.1) pondo $w=$ $u+g(\lambda)$, obtendo o problema:

$$
u_{t}=F(\lambda, u)
$$

onde $F(\lambda, u)=G(\lambda, u+g(\lambda))$ e satisfaz $F(\lambda, 0)=0 \forall \lambda$ (Rabinowitz [16]).

A solução $u \equiv 0$ é chamada de solução trivial de (3.2) e um ponto $\left(\lambda_{0}, 0\right)$ é um ponto de bifurcação para $F(\lambda, u)=0$ se em qualquer vizinhança de $\left(\lambda_{0}, 0\right)$, $F(\lambda, u)=0$ tem solução não trivial.

Definição 3.1.2: Sendo $\tilde{U}(x)$ uma solução estática de (3.2) dizemos que esta é estável numa dada norma se para qualquer $\epsilon>0$ existe $\delta>0$ tal que:

$$
\|u(x, 0)-\tilde{U}(x)\|<\delta \Rightarrow\|u(x, t)-\tilde{U}(x)\|<\epsilon \quad \forall t .
$$

Definição 3.1.3: Sendo $\tilde{U}(x)$ uma solução estática de (3.2) dizemos que esta é assintoticamente estável se:

$$
\|u(x, t)-\tilde{U}(x)\| \rightarrow 0 \quad \text { quando } t \rightarrow \infty
$$


A estabilidade da solução trivial é muitas vezes determinada através do problema linearizado. Assim linearizando (3.2) temos:

$$
v_{t}=\mathcal{L}(\lambda) v,
$$

onde $\mathcal{L}(\lambda)=F_{u}(\lambda, 0)$ é a derivada de Frechet de $F(\lambda, u)$ sobre a solução nula.

Definição 3.1.4: $O$ conjunto resolvente de $\mathcal{L}$ é o conjunto dos números complexos $\sigma$ tais que $\mathcal{L}-\sigma$ I tem inversa limitada definida em todo espaço.

Definição 3.1.5: $O$ espectro de $\mathcal{L}$ é o conjunto complementar do conjunto resolvente e o denotaremos por $\Sigma(\mathcal{L})$

Usando o teorema devido a Kielhöfer temos um critério de estabilidade para a solução trivial.

Teorema 3.1.2 ( Princípio da Estabilidade Linear): Se para $\forall \sigma \in \Sigma(\mathcal{L})$, $\sigma$ satisfaz $\operatorname{Re}(\sigma)<0$, então a solução trivial de (3.2) é assintoticamente estável. Se $\exists \sigma \in \Sigma(\mathcal{L})$ satisfazendo $\operatorname{Re}(\sigma)>0$, então a solução trivial de (3.2) é instável.

Prova: (ver Kielhöfer [11]).

Uma conseqüência imediata deste teorema é que para $\sigma$ satisfazendo $\operatorname{Re}(\sigma)=0$ ocorre mudança da estabilidade na solução nula. Logo temos que estes são pontos de bifurcação para o problema (3.2).

Se um par de autovalores complexos e conjugados cruzam o eixo imaginário, então as soluções bifurcantes podem ser periódicas em $t$, e a este fenômeno damos o nome de bifurcação de Hopf. As condições necessárias para que ocorra esse tipo de bifurcação é dado no seguinte teorema:

Teorema 3.1.3 (Bifurcação de Hopf):' Sejam $\sigma(\lambda)$ e $\bar{\sigma}(\lambda)$ autovalores do problema (3.3) que cruzam o eixo imaginário da esquerda para a direita em $\lambda=0$ tal que $\sigma(0)=\imath w_{0} \neq 0$ e $\operatorname{Re}\left(\sigma^{\prime}(0)\right)>0$. Então soluções periódicas de pequena amplitude bifurcam através da solução trivial.

Prova: (ver Britton [2]).

Escrevendo $\lambda$ como função de $u$ temos que um ponto de bifurcação pode ser representado por $\left(u^{*}, \lambda\left(u^{*}\right)\right)$. Com isto podemos classificar as bifurcações em três casos (Chow [3]): 


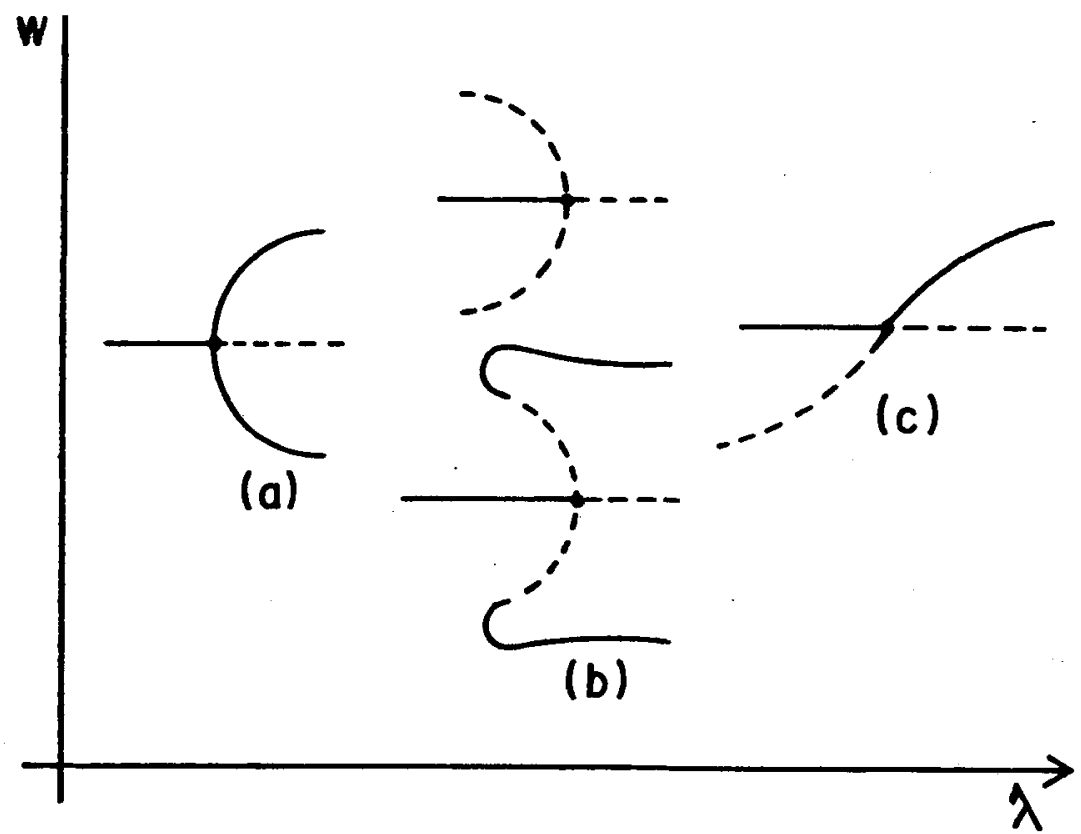

Figura 3.2: Estabilidade de soluções estáticas através de: (a) bifurcação supercrítica; (b) bifurcação subcrítica; (c) bifurcação transcrítica. Legenda: estável; - - - instável

(a) Se $\lambda^{\prime}\left(u^{*}\right)=0$ e $u \lambda^{\prime}(u)>0$ quando $u \rightarrow u^{*}$, a bifurcação é chamada de supercrítica (ver Figura 3.2-a).

(b) Se $\lambda^{\prime}\left(u^{*}\right)=0$ e $u \lambda^{\prime}(u)<0$ quando $u \rightarrow u^{*}$, a bifurcação é chamada de subcrítica (ver Figura 3.2-b).

(c) Se $\lambda^{\prime}\left(u^{*}\right) \neq 0$, a bifurcação é chamada de transcrítica (ver Figura 3.2-c).

Com o crescimento do parâmetro $\lambda$, os autovalores $\sigma$ cruzam o eixo imaginário. A solução estática originalmente estável torna-se instável.

\subsection{Problema de Reação-Difusão} satisfaça

Um problema parabólico do tipo Reação-Difusão é encontrar $u(x, t)$ que

$$
(P) \begin{cases}u_{t}=u_{x x}+\lambda f(u) & x \in(0,1), t>0 \\ u(0, t)=u(1, t)=0 & t>0 \\ u(x, 0)=u_{0}(x) & x \in[0,1]\end{cases}
$$


onde $f(u)$ geralmente é não linear e $\lambda$ é um parâmetro que pode variar num certo domínio de $\mathbf{R}$.

Analisamos as propriedades de $(P)$ considerando duas classes de funções não lineares $f(u)$, a saber:

(I) $f(u): f(0)=0, f^{\prime}(0) \neq 0, f^{\prime \prime}(0)=0$, e $f^{\prime \prime \prime}(0) \neq 0$

(II) $f(u): f(0)=f^{\prime}(0)=0$ e $f^{\prime \prime}(0) \neq 0$

O problema $(P)$ é um problema típico estudado pela Teoria da Bifurcação. Concentramos nossa análise em soluções bifurcantes da solução trivial que satisfaça $\|u\|_{\infty}<1$.

\subsection{O Problema Linearizado}

As vezes a estabilidade de soluções bifurcantes pode ser determinada através do problema linearizado. Considerando os casos (I) e (II) temos que $u \equiv 0$ é solução de $(P)$. Logo linearizando $(P)$ em $u \equiv 0$ temos o problema linear:

$$
\left\{\begin{array}{l}
v_{t}=v_{x x}+\lambda f^{\prime}(0) v \\
v(0, t)=v(1, t)=0 \\
v(x, 0)=u_{0}(x)
\end{array}\right.
$$

Resolvendo (3.4) pelo método de separação de variáveis temos que se $v(x, t)=\phi(x) \psi(t)$, então:

$$
\begin{gathered}
\psi(t)=\exp (\sigma t) \\
\left\{\begin{array}{l}
\phi_{x x}+\lambda f^{\prime}(0) \phi=\sigma \phi \\
\phi(0)=\phi(1)=0
\end{array}\right.
\end{gathered}
$$

O problema de autovalores (3.6) é fundamental para $(P)$ por dois motivos. Primeiro, a estabilidade de soluções bifurcantes pode ser determinada através do problema linearizado (3.4), e para resolver (3.4) requeremos os autovalores $\sigma$ e as autofunções $\phi(x)$ para um $\lambda$ fixo. Segundo, para determinar os pontos onde soluções estáticas bifurcam da solução trivial, precisamos dos autovalores $\sigma$ da derivada de Frechet de $(P)$ sobre a solução trivial determinados por (3.6). Como conseqüência do Teorema 3.1.2, temos que para $\sigma$ satisfazendo $\operatorname{Re}(\sigma)=0$ ocorrem bifurcações para $(P)$ através da solução nula. Stuart [22] prova através do teorema seguinte que $\operatorname{Im}(\sigma)=0$. 
Teorema 3.3.1: Os autovalores $\sigma$ do problema (3.6) são reais.

Prova: Multiplicando (3.6) por $\bar{\phi}(x)$ e integrando em [0,1] temos

$$
\begin{array}{r}
\int_{0}^{1} \bar{\phi}\left(\phi_{x x}+\lambda f^{\prime}(0) \phi-\sigma \phi\right) d x=0 \Rightarrow \\
\int_{0}^{1}\left(\bar{\phi} \phi_{x x}+\lambda f^{\prime}(0) \bar{\phi} \phi-\sigma \bar{\phi} \phi\right) d x=0 \Rightarrow \\
\left(\lambda f^{\prime}(0)-\sigma\right) \int_{0}^{1} \bar{\phi} \phi d x+\left[\left.\bar{\phi} \phi_{x}\right|_{0} ^{1}\right]-\int_{0}^{1} \bar{\phi}_{x} \phi_{x} d x=0 \Rightarrow \\
\left(\sigma-\lambda f^{\prime}(0)\right) \int_{0}^{1}|\phi|^{2} d x+\int_{0}^{1}\left|\phi_{x}\right|^{2} d x=0
\end{array}
$$

Desde que $\lambda \in \mathbf{R}$ a parte imaginária da equação é dada por:

$$
\operatorname{Im}(\sigma) \int_{0}^{1}|\phi|^{2} d x=0
$$

$\log \circ \operatorname{Im}(\sigma)=0$

Aplicando o mesmo método de linearização de $(P)$ sobre qualquer solução estática, conclui-se que $\operatorname{Im}(\sigma)=0$. Isto significa que o problema $(P)$ não satisfaz às condições do Teorema 3.1.3, portanto bifurcações do tipo Hopt não ocorrem, ou seja, $(P)$ não exibe soluções periódicas.

\subsection{Análise das Bifurcações}

Nesta seção analisamos a bifurcação de soluções estáticas de $(P)$ através de $u(x, t) \equiv 0$. Dos Teoremas (3.1.2) e (3.3.1) temos que bifurcações para $(P)$ ocorrem quando $\sigma=0$. Assim do Teorema da Função Implícita, temos que $\left(\lambda_{n}, 0\right)$ são pontos de bifurcação para $(P)$ se, e somente se, $\lambda_{n}$ são autovalores de (3.6) $\operatorname{com} \sigma=0$. O problema (3.6) com $\sigma=0$ não possui autofunções no caso (II), assim vamos considerar apenas o caso (I) e sem perda de generalidade assumimos $f^{\prime}(0)=1$. Por motivação consideramos o seguinte argumento para a obtenção de (3.6). Desejamos soluções estáticas $\tilde{U}(x)$ de $(P)$ com $\|\tilde{U}\|_{\infty} \ll 1$ da forma:

$$
\tilde{U}(x) \approx \varepsilon \phi(x)
$$

onde $\varepsilon \ll 1$. Substituindo em $(P)$ temos $\varepsilon \phi_{x x}+\lambda f(\varepsilon \phi)=0$ e linearizando obtemos: $\phi_{x x}+\lambda f^{\prime}(0) \phi=0$ como em (3.6).

Desta forma, para que (3.6) tenha solução da forma (3.7), vamos exigir que (3.6) possua solução não trivial. O problema $(3.6)$ com $f^{\prime}(0)=1$, é idêntico 
ao problema $(1.3-1.4)$ mostrado no capitulo 1 com $L=1$. Logo temos que:

$$
\lambda_{s}=(s \pi)^{2} \text { e } \phi_{s}(x)=\operatorname{sen}(s \pi x)
$$

com $s$ inteiro não nulo, são respectivamente os autovalores e autofunções de (3.6) com $\sigma=0$. Logo $\lambda_{s}=(s \pi)^{2}$ são pontos onde bifurcam soluções estáticas da solução trivial. Assim de (3.7) temos $\tilde{U}(x) \approx \varepsilon a_{s} \operatorname{sen}(s \pi x)$ para $\lambda \approx(s \pi)^{2}$ onde os $a_{s}$ ainda são indeterminados.

Vamos considerar a próxima ordem da expansão para $\lambda \approx \pi^{2}$. Sendo $f^{\prime \prime}(0)=0$ não existe o termo de $\mathcal{O}\left(\varepsilon^{2}\right)$ na expressão de $\tilde{U}(x)$, assim consideremos:

$$
\tilde{U}(x) \approx \varepsilon a_{1} \phi_{1}(x)+\varepsilon^{3} \chi(x) \text { e } \lambda \approx \pi^{2}+\lambda_{0} \varepsilon^{2} .
$$

Substituindo em $(P)$ e expandindo $f(u)$ em série de Taylor obtemos:

$$
\varepsilon a_{1} \phi_{1 x x}+\varepsilon^{3} \chi_{x x}+\left(\pi^{2}+\lambda_{0} \varepsilon^{2}\right)\left[\varepsilon a_{1} \phi_{1}+\varepsilon^{3} \chi+\frac{f^{\prime \prime \prime}(0)}{6}\left(\varepsilon a_{1} \phi_{1}+\varepsilon^{3} \chi\right)^{3}+\ldots\right]=0
$$

Considerando os termos de $\mathcal{O}\left(\varepsilon^{3}\right)$ e impondo as condições de contorno produz:

$$
\left\{\begin{array}{l}
\chi_{x x}+\pi^{2} \chi=-\lambda_{0} a_{1} \phi_{1}-\pi^{2} \frac{f^{\prime \prime \prime}(0)}{6} \phi_{1}{ }^{3} a_{1}{ }^{3} \\
\chi(0)=\chi(1)=0
\end{array}\right.
$$

A versão homogênea de (3.9) é igual à versão homogênea de (3.6) com $\lambda=\pi^{2}$, logo este é singular. Então para que (3.9) tenha solução, esta deve satisfazer a Alternativa de Fredholm ( ver Iooss [10]), ou seja (3.9) possui solução se, e somente se:

$$
\left\langle-\lambda_{0} a_{1} \phi_{1}-\pi^{2} \frac{f^{\prime \prime \prime}(0)}{6} a_{1}{ }^{3} \phi_{1}{ }^{3}, \phi_{1}\right\rangle=0
$$

com isto temos que :

$$
\left\{\begin{array}{rl}
a_{1} & =0 \\
& \text { ou } \\
a_{1}{ }^{2} & =\frac{-6 \lambda_{0} \int_{0}^{1} \phi_{1}{ }^{2} d x}{\pi^{2} f^{\prime \prime \prime}(0) \int_{0}^{1} \phi_{1}{ }^{4} d x}
\end{array} .\right.
$$

Resultados similares são encontrados para $\lambda \approx(s \pi)^{2}+\lambda_{0} \varepsilon^{2}$, onde os coeficientes $a_{s}$ são dados por:

$$
\left\{\begin{array}{rl}
a_{s} & =0 \\
& \text { ou } \\
a_{s}^{2} & =\frac{-6 \lambda_{0} \int_{0}^{1} \phi_{s}{ }^{2} d x}{s^{2} \pi^{2} f^{\prime \prime \prime}(0) \int_{0}^{1} \phi_{s}{ }^{4} d x}
\end{array} .\right.
$$

Para analisarmos a estrutura do diagrama de birfucação vamos considerar os seguintes casos: 


\section{$\|U\|_{\infty}$}

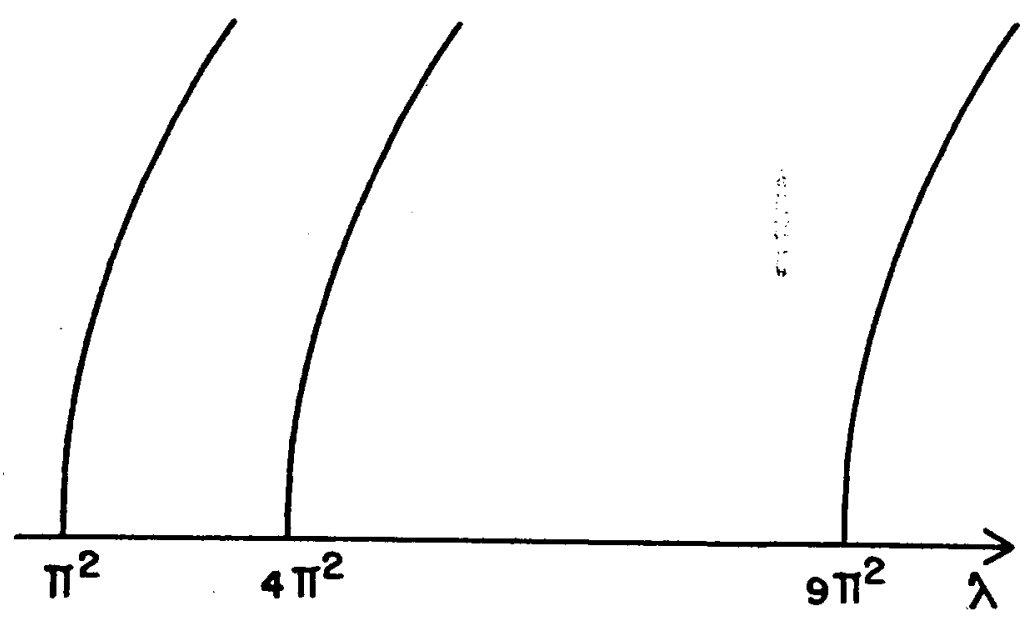

Figura 3.3: Diagrama de Bifurcação para $(P)$ quando $f^{\prime \prime \prime}(0)<0$.

i) Se $f^{\prime \prime \prime}(0)<0$, então $\lambda_{0}>0$, logo $\lambda=\pi^{2}+\varepsilon^{2} \lambda_{0}$ é uma função crescente de $\lambda_{0}$ fornecendo o diagrama de bifurcação mostrado na Figura 3.3.

ii) Se $f^{\prime \prime \prime}(0)>0$, então $\lambda_{0}<0, \log \lambda_{=}=\pi^{2}+\varepsilon^{2} \lambda_{0}$ é uma função descrescente de $\lambda_{0}$ fornecendo o diagrama de bifurcação mostrado na Figura 3.4 .

Com o Teorema 3.1.2, podemos deduzir as propriedades das soluçóes estáticas de $(P)$ através do problema linearizado. Como visto na seção 3.3 a solução do problema linearizado depende da solução de (3.5) e (3.6). Assumindo que $f^{\prime}(0)=1$, de (3.6) temos que $\phi_{x x}+(\lambda-\sigma) \phi=0$, logo $\phi(x)=\operatorname{sen}(s \pi x)$ e $\sigma=\lambda-s^{2} \pi^{2}$. Assim a solução geral do problema $(P)$ linearizado é dado por:

$$
v(x, t)=\sum_{s=1}^{\infty} a_{s} \exp \left[\left(\lambda-\pi^{2} s^{2}\right) t\right] \operatorname{sen}(s \pi x),
$$

onde os $a_{s}$ são determinados pelas condições iniciais em (3.4). Para $\lambda<\pi^{2}$ temos que $v(x, t) \rightarrow 0$ quando $t \rightarrow \infty$ e para $\lambda>\pi^{2}$ temos $v(x, t) \rightarrow \infty$ quando $t \rightarrow \infty$.

Usando o Teorema 3.1.2, podemos fazer as seguintes afirmações sobre $(P): u(x, t) \equiv 0$ é solução estável de $(P)$ para $\lambda<\pi^{2}$ e instável para $\lambda>\pi^{2}$. O valor $\lambda=\pi^{2}$ é o primeiro ponto de bifurcação através de $u \equiv 0$ ( onde $\sigma=0$ ). Conseqüentemente o primeiro ponto de bifurcação proveniente de 


\section{$\|U\|_{\infty}$}

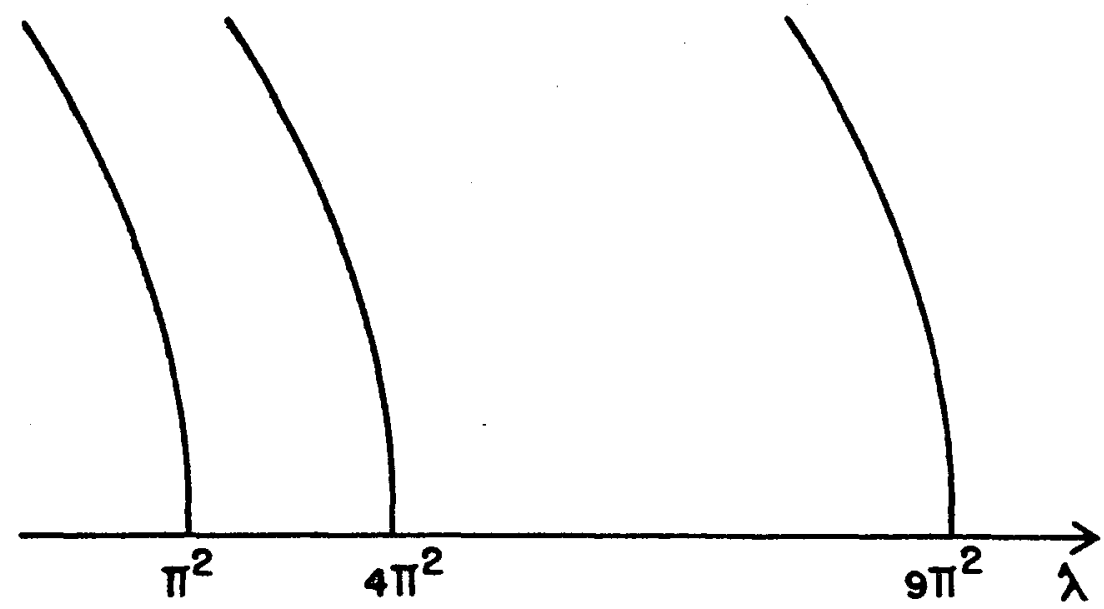

Figura 3.4: Diagrama de Bifurcação para $(P)$ quando $f^{\prime \prime \prime}(0)>0$.

$u \equiv 0$ é o ponto de mudança de estabilidade da solução trivial. Desta forma, uma análise puramente linear determina a estabilidade da solução para perturbações infinitamente pequenas.

\subsection{Análise Não Linear Fraca}

Para dizer algo a mais da dinâmica de $(P)$, devemos levar em consideração a forma não linear de $f(u)$. Faremos assim uma " análise não linear fraca" de $(P)$ para $\lambda \approx \pi^{2}$. A essência da análise não linear fraca é considerar uma modulação da solução linearizada para $\lambda=\pi^{2}$. A modulação é escolhida em uma escala de tempo reduzida, na qual, o efeito da não linearidade em $f(u)$ é pronunciado. Aplicaremos aqui o método de múltiplas escalas, onde consideramos três regiões de tempo (Matkowsky [13]). A primeira região é definida onde a solução começa a evoluir dos dados iniciais. Aqui esperamos que a teoria linear seja válida. A segunda região é aquela, na qual os efeitos da não linearidade começam a fazer efeito. A solução continua se desenvolvendo no tempo até aproximar-se do estado estático onde se forma a terceira região.

Formalmente fazemos $\lambda=\pi^{2}+\lambda_{0} \varepsilon^{2}$ e procuramos uma expansão para 
$u(x, t)$ na forma:

$$
u(x, t ; \varepsilon) \approx \sum_{m=1}^{\infty} v_{m}(x, t, \tau) \varepsilon^{m}
$$

onde $\tau=\varepsilon^{2} t$.

Suponhamos que o dado inicial é de pequena amplitude, isto é:

$$
u(x, 0)=\varepsilon g(x)
$$

De acordo com o método das múltiplas escalas, tratamos $t$ e $\tau$ como variáveis independentes e abusando da notação colocamos:

$$
\frac{\partial}{\partial t} \equiv \frac{\partial}{\partial t}+\varepsilon^{2} \frac{\partial}{\partial \tau}
$$

Substituindo (3.14) e (3.12) em $(P)$ e sendo $f(u)$ pertencente ao caso (I) temos:

$$
\begin{aligned}
\sum_{m=1}^{\infty}\left(v_{m}\right)_{t} \varepsilon^{m}+\sum_{m=1}^{\infty}\left(v_{m}\right)_{\tau} \varepsilon^{m+2}= & \sum_{m=1}^{\infty}\left(v_{m}\right)_{x x} \varepsilon^{m}+\left(\pi^{2}+\varepsilon^{2} \lambda_{0}\right)\left[\sum_{m=1}^{\infty} v_{m} \varepsilon^{m}+\right. \\
& \left.\frac{f^{\prime \prime \prime}(0)}{6}\left(\sum_{m=1}^{\infty} v_{m} \varepsilon^{m}\right)^{3}+\ldots\right]
\end{aligned}
$$

Igualando termo a termo a série de potência em $\varepsilon$ obtemos:

$$
\begin{aligned}
& \mathcal{L} v_{1}=0 \\
& \mathcal{L} v_{2}=0 \\
& \mathcal{L} v_{3}=-\left(v_{1}\right)_{\tau}+\lambda_{0} v_{1}+\pi^{2} \frac{f^{\prime \prime \prime}(0)}{6} v_{1}^{3}=r_{3} .
\end{aligned}
$$

onde $\mathcal{L} v=v_{t}-v_{x x}-\pi^{2} v$. Da equação (3.13) temos $v_{1}(x, 0)=g(x)$ e $v_{m}(x, 0)=0$ para $m>1$. A equação (3.15), junto com a condição de fronteira, tem solução dada por (3.11) com $\lambda=\pi^{2}$. Os coeficientes $a_{s}$ são funções de $\tau$, ainda não determinadas, desta forma:

$$
v_{1}(x, t)=a_{1}(\tau) \operatorname{sen}(\pi x)+\sum_{s=2}^{\infty} a_{s}(\tau) \exp \left[\left(1-s^{2}\right) \pi^{2} t\right] \operatorname{sen}(s \pi x)
$$

onde a expresão dentro da soma decai com o tempo.

A solução para $v_{2}$ é a solução nula, pois $\mathcal{L}$ é um operador linear e as condições iniciais e de fronteira são homogêneas. 
Desde que o mesmo operador $\mathcal{L}$ ocorre em sucessivas ordens, soluçóes seculares podem surgir. A condição necessária e suficiente para que o problema (3.17) tenha solução limitada (Matkowsky [13])é que:

$$
\lim _{T \rightarrow \infty} \frac{1}{T} \int_{0}^{T} \int_{0}^{1} \exp \left[\left(1-s^{2}\right) \pi^{2} t\right] \operatorname{sen}(s \pi x) r_{3} d x d t=0 ; s=1,2, \ldots
$$

A única contribuição para (3.19) ocorre com $s=1$, e surge do primeiro termo em (3.18). Quando substituimos este termo em $r_{3}$ obtemos:

$$
\begin{array}{r}
\lim _{\tau \rightarrow \infty} \frac{1}{T} \int_{0}^{T} \int_{0}^{1} \operatorname{sen}(\pi x)\left(-\frac{d a_{1}(\tau)}{d \tau} \operatorname{sen}(\pi x)+\lambda_{0} a_{1}(\tau) \operatorname{sen}(\pi x)+\right. \\
\left.\pi^{2} \frac{f^{\prime \prime \prime}(0)}{6} a_{1}^{3}(\tau) \operatorname{sen}^{3}(\pi x)\right) d x d t=0 .
\end{array}
$$

Com isto obtemos a seguinte equação diferencial ordinária:

$$
\frac{d a_{1}(\tau)}{d \tau}=\lambda_{0} a_{1}(\tau)+b a_{1}{ }^{3}(\tau)
$$

onde

$$
b=\frac{\pi^{2} f^{\prime \prime \prime}(0) \int_{0}^{1} \operatorname{sen}^{4}(\pi x) d x}{6 \int_{0}^{1} \operatorname{sen}^{2}(\pi x) d x}
$$

Os pontos críticos de (3.20) são dados por $a_{1}=0$ ou $a_{1}= \pm\left(-\lambda_{0} / b\right)^{1 / 2}$. Estes pontos críticos são precisamente a amplitude das soluções estáticas dadas por (3.10). Considerando $a_{1}(\tau) \neq 0,(3.20)$ tem solução geral dada por:

$$
a_{1}(\tau)= \pm\left(\frac{\lambda_{0} \exp \left(2 \lambda_{0} \tau\right)}{C \lambda_{0}-b \exp \left(2 \lambda_{0} \tau\right)}\right)^{1 / 2}
$$

onde $C$ é uma constante. Combinando (3.12) e (3.18) temos que $u(x, t) \approx$ $\varepsilon a_{1}(\tau) \operatorname{sen}(\pi x)$ quando $t \rightarrow \infty$. Assim podemos conhecer o comportamento de $u(x, t)$ estudando o comportamento de $a_{1}(\tau)$. Considerando a condição inicial para o problema (3.21) dada por $a_{1}(0)$ obtemos:

$$
C=\frac{b}{\lambda_{0}}+\frac{1}{a_{1}^{2}(0)}
$$

Para estudar o comportamento de $a_{1}(\tau)$ devemos considerar os seguintes casos: 


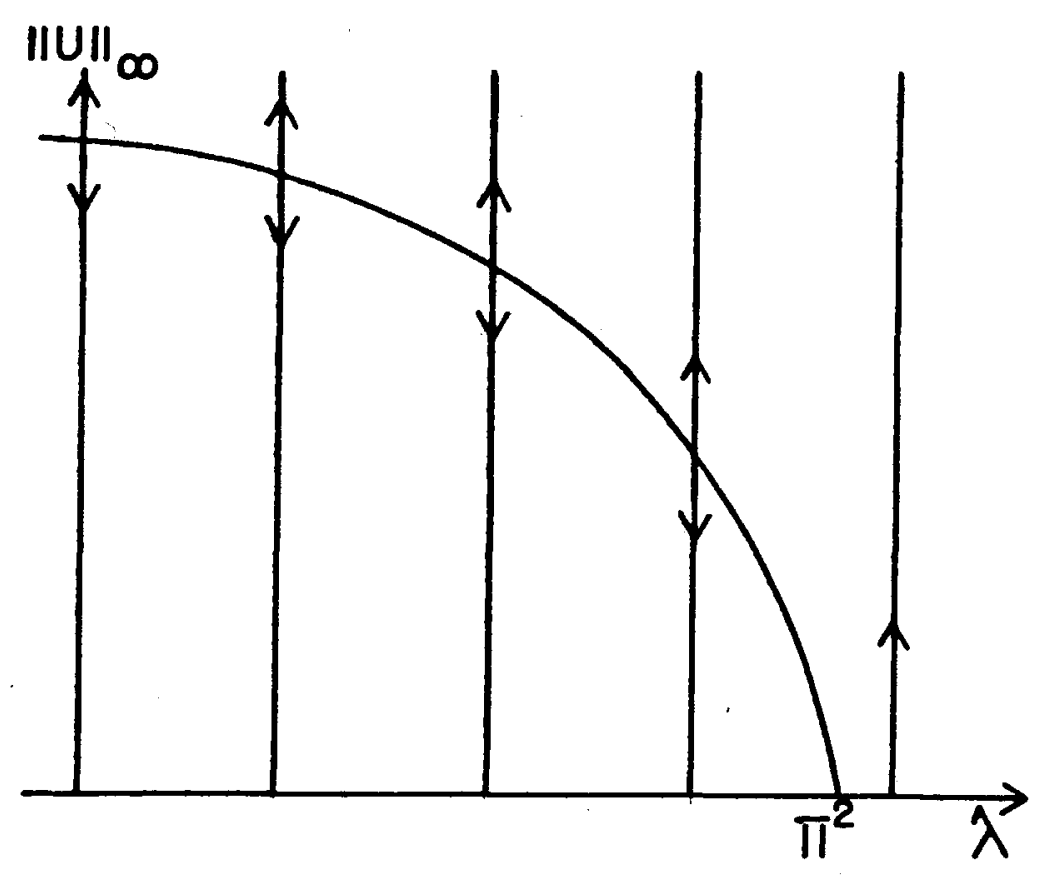

Figura 3.5: Diagrama mostrando a evolução dos dados sobre $(P)$ quando $f^{\prime \prime \prime}(0)>0$. As flechas indicam a evolução no tempo.

(a) Para $\lambda_{0}>0$ e $b>0$ temos por (3.22) que $C>0$. Então para que exista solução real em (3.21) é necessário que $\lambda_{0} C-b \exp \left(2 \lambda_{0} \tau\right)>0$. Como $\exp \left(2 \lambda_{0} \tau\right)$ é uma função crescente, então existe $\tau_{i}$ tal que :

$$
\lambda_{0} C-b \exp \left(2 \lambda_{0} \tau_{i}\right)=0
$$

e portanto $a_{1}(\tau) \rightarrow \infty$ quando $\tau \rightarrow \tau_{i}$. Logo concluimos que a solução nula é instável como previsto pela da teoria linear (Figura $3.5 \operatorname{com} \lambda>\pi^{2}$ ).

(b) Para $\lambda_{0}<0$ e $b>0$ temos que existirá solução real se $\lambda_{0} C-b \exp \left(2 \lambda_{0} \tau\right)<0$, para $\forall \tau$, isto é satisfeito com $C>0$, logo de (3.22) obtemos:

$$
\frac{b}{\lambda_{0}}+\frac{1}{a_{1}^{2}(0)}>0 \Rightarrow\left|a_{1}(0)\right|<\left(\frac{-\lambda_{0}}{b}\right)^{1 / 2} \text {. }
$$

Assim $a_{1}(\tau) \rightarrow 0$ quando $\tau \rightarrow \infty$. Para valores iniciais $\left|a_{1}(0)\right|>\left(-\lambda_{0} / b\right)^{1 / 2}$ temos que $a_{1}(\tau) \rightarrow \infty$ quando $\tau \rightarrow \infty$ (ver Figura $3.5 \operatorname{com} \lambda<\pi^{2}$ ).

Este é um exemplo de amplitude finita instável. A solução nula de $(P)$ é instável para perturbações satisfazendo $-a_{1}(0)+>\left(-\lambda_{0} / b\right)^{1 / 2}$ 


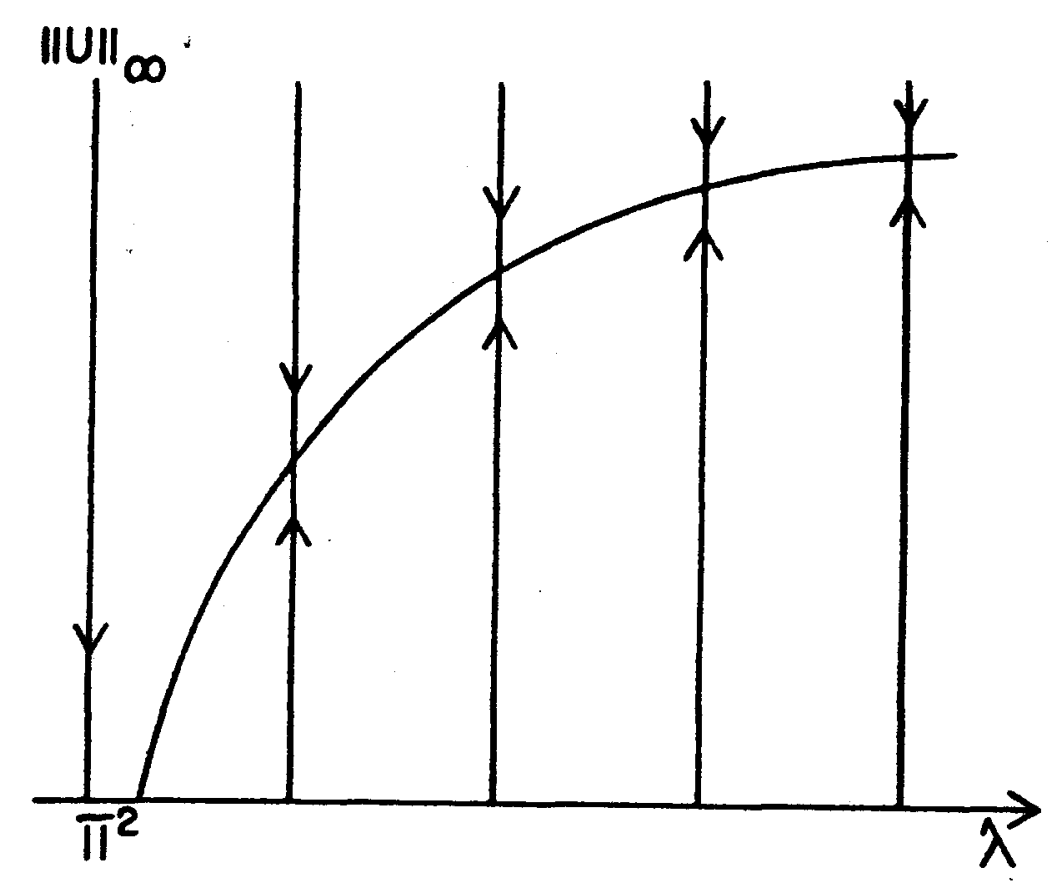

Figura 3.6: Diagrama mostrando a evolução dos dados sobre $(P)$ quando $f^{\prime \prime \prime}(0)<0$. As flechas indicam a evolução no tempo.

(c) Para $\lambda_{0}>0$ e $b<0$ existirá solução real de (3.21) para qualquer valor de $C$. Quando $\tau \rightarrow \infty$ temos que:

$$
a_{1}(\tau) \rightarrow \pm\left(\frac{-\lambda_{0}}{b}\right)^{1 / 2}
$$

Desta forma a solução nula é instável como previsto na teoria linear ( ver Figura 3.6, $\operatorname{com} \lambda>\pi^{2}$ ).

(d) Para $\lambda_{0}<0$ e $b<0$ temos que $C>0$, logo existirá solução real para qualquer valor de $C$. Quando $\tau \rightarrow \infty$ temos que $a_{1}(\tau) \rightarrow 0$ confirmando que a solução nula é estável para $\lambda_{0}<0$ (ver Figura 3.6, $\operatorname{com} \lambda<\pi^{2}$ ). 


\subsection{Bifurcação no Infinito}

Para $\lambda \rightarrow \infty$ pode existir soluções que convergem para a solução trivial. Neste caso $\lambda=\infty$ é considerado como ponto de bifurcação e este fenômeno é conhecido como bifurcação no infinito.

Vamos considerar por simplicidade, que $f(u)=u^{2}$ ( um caso particular do caso (II) ). Fazendo uma mudança de escala da forma $\tau=\lambda t$ e substituindo em $(P)$ obtemos:

$$
\left\{\begin{array}{l}
u_{\tau}=\lambda^{-1} u_{x x}+u^{2} \\
u(0, \tau)=u(1, \tau)=0
\end{array}\right.
$$

juntamente com uma condição inicial. Desde que $f^{\prime}(0)=0$ deduzimos que não existe bifurcação através da solução trivial, pois de (3.6) com $\sigma=0$ temos que $\phi_{x x}=0 \operatorname{com} \phi(1)=\phi(0)=0$ e consequëntemente $\phi(x)=0$.

Linearizando (3.23) sobre $u(x, \tau) \equiv 0$ obtemos:

$$
v_{\tau}=\lambda^{-1} v_{x x} .
$$

Resolvendo a equação acima, por separação de variáveis, obtemos a solução geral linearizada dada por:

$$
v(x, \tau)=\sum_{s=0}^{\infty} a_{s} \exp \left(\frac{-s^{2} \pi^{2}}{\lambda} \tau\right) \operatorname{sen}(s \pi x)
$$

Para $f(u)$ como no caso (I) os pontos de bifurcação $\lambda=s^{2} \pi^{2}$ são definidos como aqueles onde a taxa de crescimento das componentes individuais $\operatorname{sen}(s \pi x)$ que compõem a solução linearizada (3.11) são independentes da variável tempo ( pois $\sigma=0$ ). Neste sentido o ponto $\lambda=\infty$ é um candidato a ponto de bifurcação de multiplicidade infinita no problema do caso (II), pois a taxa de crescimento de cada componente em (3.24) se aproxima de zero quando $\lambda \rightarrow \infty$.

Esta discussão heurística não constitui uma prova. De qualquer modo no caso em que $f(u)=u^{2}$, pode-se mostrar que (3.23) possui uma solução estática da forma $\tilde{U}(x)=\lambda^{-1} \tilde{U}_{1}(x)$ não trivial (ver Rosemblat [18]). Para o nosso propósito, é suficiente notar que a bifurcação no infinito, é caracterizada pelo caso onde a taxa de crescimento da solução linearizada aproxima-se de zero. Desta forma a bifurcação é subcrítica e a solução é instável. Assim, uma instabilidade de amplitude finita ocorre neste problema, mesmo que não ocorra bifurcação através da solução trivial para qualquer valor finito de $\lambda$. 


\section{Capítulo 4}

\section{Discretização da Equação Reação-Difusão}

Neste capítulo analisamos a estabilidade numérica da discretização da equação Reação-Difusão. Iniciamos com a discretização do problema $(P)$ através dos métodos de Crank-Nicolson e analisamos o problema discreto resultante, valendo-se de idéias similares àquelas utilizadas no capítulo anterior para o caso contínuo.

\subsection{O Problema Discreto} obtemos:

Usando os métodos de Crank-Nicolson para discretizar o problema $(P)$

$$
(P D)\left\{\begin{array}{l}
u_{j}^{n+1}-u_{j}^{n}=r \delta_{x}^{2}\left[\theta u_{j}^{n+1}+(1-\theta) u_{j}^{n}\right]+\lambda h\left[\theta f\left(u_{j}^{n+1}\right)+(1-\theta) f\left(u_{j}^{n}\right)\right] \\
u_{0}^{n}=u_{J}^{n}=0 \\
u_{j}^{0}=u_{0}(j k) \text { para } j=1,2, \ldots, J-1
\end{array}\right.
$$

onde $J k=1, r=h / k^{2}$ e $0 \leq \theta \leq 1$.

Faremos primeiro a análise da estabilidade absoluta de $(P D)$ (Definição 2.8.1). e então através de uma análise similar a do problema $(P)$, mostraremos que a instabilidade numérica não-linear está associada com a bifurcação de soluções periódicas em $n$. Ao contrário do problema contínuo, o comportamento global de $(P D)$ é desconhecido. A dinâmica da discretização é geralmente mais complicada que a dinâmica do respectivo problema contínuo. Um importante trabalho de Hoff [8] generaliza o conceito de regiões invariantes para o problema discreto. 
O método pode ser usado para provar resultados globais sobre a discretização de equações de Reação-Difusão específicas.

\subsection{O Problema Linearizado}

$$
\begin{gathered}
\text { A linearização de }(P D) \text { sobre } u_{j}^{n} \equiv 0 \text { é dado por: } \\
\left\{\begin{array}{l}
v_{j}^{n+1}-v_{j}^{n}=r \delta_{x}^{2}\left[\theta v_{j}^{n+1}+(1-\theta) v_{j}^{n}\right]+\lambda h f^{\prime}(0)\left[\theta v_{j}^{n+1}+(1-\theta) v_{j}^{n}\right] \\
v_{0}^{n}=v_{J}^{n}=0 \\
v_{j}^{0}=u_{0}(j k) \quad \text { para } j=1,2, \ldots, J-1
\end{array}\right.
\end{gathered}
$$

Como no caso contínuo, este problema pode ser resolvido por separação de variáveis. Vamos procurar soluções da forma $v_{j}^{n}=\phi_{j} \psi_{n}$. Substituindo em (4.1) obtemos:

$$
\frac{\psi_{n+1}-\psi_{n}}{\theta \psi_{n+1}+(1-\theta) \psi_{n}}=\sigma=\frac{r \delta_{x}^{2} \phi_{j}+\lambda h f^{\prime}(0) \phi_{j}}{\phi_{j}}
$$

que nos fornece as seguintes equações diferenças:

$$
\begin{gathered}
\psi_{n}=\left[\frac{1+\sigma(1-\theta)}{1-\sigma \theta}\right]^{n} ; \\
\left\{\begin{array}{l}
r \delta_{x}^{2} \phi_{j}+\lambda h f^{\prime}(0) \phi_{j}=\sigma \phi_{j} \quad j=1,2, \ldots, J-1 \\
\phi_{0}=\phi_{J}=0
\end{array} .\right.
\end{gathered}
$$

As equações (4.2) e (4.3) são as discretizações das equações (3.5) e (3.6) respectivamente, e são fundamentais para $(P D)$. Na seção 3.3 vimos que bifurcações em $(P)$ ocorrem onde a solução do problema linear é independente do tempo. De forma similar Stuart [22] descreve bifurcação em sistemas discretos. Com a condição $|\xi|=1$ temos que (4.2) é neutra em $n$, onde:

$$
\xi=\frac{1+\sigma(1-\theta)}{1-\sigma \theta}
$$

O teorema que segue é uma versão discreta do Teorema 3.3.1. (Stuart [22]).

Teorema 4.2.1: Os autovalores $\sigma$ do problema (4.3) são reais.

Prova: Multiplicando (4.3) por $\bar{\phi}_{j}$ o complexo conjugado de $\phi_{j}$ e somando por partes (Richtmyer [17] pag. 14) obtemos:

$$
\sum_{j=1}^{J-1}\left[r \delta_{x}^{2} \phi_{j}+\lambda h f^{\prime}(0) \phi_{j}\right] \bar{\phi}_{j}=\sum_{j=1}^{J-1} \sigma \phi_{j} \bar{\phi}_{j} \Rightarrow
$$




$$
\begin{array}{r}
r \sum_{j=1}^{J-1}\left(\phi_{j-1}-2 \phi_{j}+\phi_{j+1}\right) \bar{\phi}_{j}+\left[\lambda h f^{\prime}(0)-\sigma\right] \sum_{j=1}^{J-1} \phi_{j} \bar{\phi}_{j}=0 \Rightarrow \\
\left(\phi_{J}-\phi_{J-1}\right) \bar{\phi}_{J}-\left(\phi_{1}-\phi_{0}\right) \bar{\phi}_{1}-r \sum_{j=1}^{J-1}\left(\phi_{j+1}-\phi_{j}\right)\left(\phi_{j+1}-\bar{\phi}_{j}\right)+ \\
{\left[\lambda h f^{\prime}(0)-\sigma\right] \sum_{j=1}^{J-1} \phi_{j} \bar{\phi}_{j}=0 \Rightarrow} \\
r \sum_{j=1}^{J-1}\left|\phi_{j+1}-\phi_{j}\right|^{2}+\left[\sigma-\lambda h f^{\prime}(0)\right] \sum_{j=1}^{J-1}\left|\phi_{j}\right|^{2}+\left|\phi_{1}\right|^{2}=0 .
\end{array}
$$

Sendo $\lambda$ real a parte imaginária da equação acima é dada por:

$$
\operatorname{Im}(\sigma) \sum_{j=1}^{J-1}\left|\phi_{j}\right|^{2}=0
$$

logo temos que $\operatorname{Im}(\sigma)=0$.

Sendo $\sigma$ real temos que $\xi$ é real, conseqüentemente as bifurcações ocorrem para $\xi= \pm 1$. Quando $\xi=1$ temos de (4.4) que $\sigma=0$, o que corresponde às bifurcações estáticas como no caso contínuo. $\mathrm{O}$ caso em que $\xi=-1$ não tem nenhuma analogia com o caso contínuo. Este fenômeno é conhecido como " flip bifurcation point ". Neste caso as soluções bifurcantes tem período $2 \mathrm{em} n$, sendo um produto da discretização. A condição de $\xi=-1$ corresponde no problema linear o inicio da instabilidade numérica (ver Figura 2.5). No caso não linear de $(P D)$, a instabilidade está associada com a bifurcação de soluções periódicas de $(P D)$ através da solução trivial. Os valores críticos onde estas bifurcações ocorrem são determinados pela teoria linear. Como no problema $(P)$, o comportamento de $(P D)$ depende das propriedades das soluções bifurcantes. Esta situação é mais complicada que o caso contínuo, pois necessitamos considerar não apenas as bifurcações de soluções estáticas, mas também as bifurcações de soluções periódicas que afetam a estabilidade numérica do sistema.

\subsection{Análise das Bifurcações}

Consideremos $f(u)$ pertencente ao caso (I). Resolvendo o problema de autovalores (4.3), determinamos os pontos de bifurcação de $(P D)$ através da solução trivial $u_{j}^{n} \equiv 0$. Vamos procurar soluções da forma:

$$
\phi_{j}=\exp \left(\frac{i s \pi j}{J}\right)
$$


onde $s$ é inteiro. Substituindo em (4.3) obtemos:

$$
2 r \exp \left(\frac{i s \pi j}{J}\right)\left[\cos \left(\frac{s \pi}{J}\right)-1\right]=\left[\sigma-\lambda h f^{\prime}(0)\right] \exp \left(\frac{i s \pi j}{J}\right) .
$$

Desta forma temos que:

$$
\sigma=-4 r \operatorname{sen}^{2}\left(\frac{s \pi}{2 J}\right)+\lambda h f^{\prime}(0) .
$$

Impondo as condições de contorno temos que $\phi_{j}$ só deve ser formado pela parte imaginária, ou seja :

$$
\phi_{j}=\operatorname{sen}\left(\frac{s \pi j}{J}\right)
$$

Para o propósito de representação numa malha discreta necessitamos apenas considerar $s=1,2, \ldots, J-1$. Substituindo (4.5) em (4.4) obtemos:

$$
\xi_{s}=\frac{1-4 r(1-\theta) \operatorname{sen}^{2}\left(\frac{s \pi}{2 J}\right)+\lambda h f^{\prime}(0)(1-\theta)}{1+4 r \theta \operatorname{sen}^{2}\left(\frac{s \pi}{2 J}\right)+\lambda h f^{\prime}(0) \theta} ; s=1,2, \ldots, J-1
$$

A condição $\xi_{s}=1$ define os pontos de bifurcação de soluções estáticas, e a condição $\xi_{s}=-1$ define os pontos de bifurcação de soluções periódicas. Vamos comparar os pontos de bifurcação de soluções estáticas de $(P D)$ com os pontos de $(P)$. Considerando $\xi_{s}=1$ e $f^{\prime}(0)=1$, temos de (4.7) que:

$$
\lambda_{s}=\frac{4}{k^{2}} \operatorname{sen}^{2}\left(\frac{s \pi}{2 J}\right) ; s=1,2, \ldots, J-1
$$

O modelo discreto possui apenas $(J-1)$ pontos de bifurcação de soluções estáticas, enquanto que ó modelo contínuo $(P)$ possui um número infinito (ver (3.8)). Comparando as autofunções em (3.8) com (4.6) vemos que estas são iguais nos pontos $x=j / J=j k$. Expandindo (4.8) em torno de $s=0$ onde $k<s \ll J=k^{-1}$ temos que:

$$
\lambda_{s} \approx \pi^{2} s^{2}\left[1-\left(\frac{s \pi k}{12}\right)^{2}\right]
$$

Comparando estes resultados com (3.8) deduzimos que para $s \ll J$ os autovalores são aproximados com erro de $\mathcal{O}\left(k^{2}\right)$. Considerando a expansão de (4.8) em torno de $s=J$, onde $(J-s) / J \ll 1$, obtemos:

$$
\lambda_{s} \approx \frac{4}{k^{2}}\left[1-\pi^{2} \frac{(J-s)^{2}}{8 J^{2}}\right] \text {. }
$$




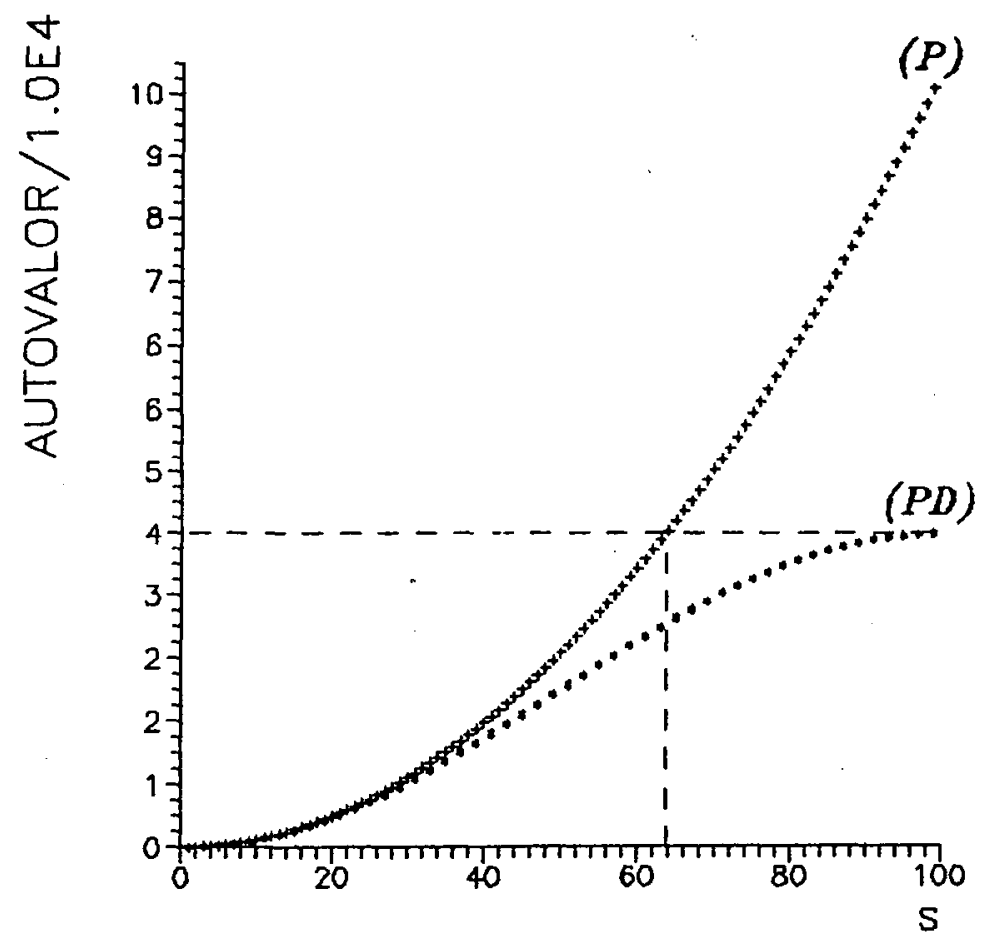

Figura 4.1: Comparação dos autovalores de $(P) \operatorname{com}(P D)$ para $k=0.01$.

Esta equação possui uma pequena semelhança com (3.8). Na figura (4.1) mostramos um gráfico comparativo dos pontos de bifurcação de $(P)$ e $(P D)$ com $k=0.01$. O gráfico confirma a análise acima e mostra que os primeiros pontos de bifurcação em $(P)$ são bem representados por $(P D)$.

É possivel construir soluções estáticas não triviais de $(P D)$, numa vizinhança dos pontos de bifurcação dados por (4.8). A análise discreta é análoga à feita na seção 3.3 , ou seja, procuramos uma expansão da forma:

$$
\tilde{U}_{j} \approx \varepsilon a_{s} \operatorname{sen}\left(\frac{s \pi j}{J}\right)+\varepsilon^{3} \chi_{j} ; \lambda \approx \lambda_{s}+\lambda_{0} \varepsilon^{2} .
$$

Sem perda de generalidade consideremos $f^{\prime}(0)=1$. Substituindo (4.9) em $(P D)$ obtemos:

$$
\begin{array}{r}
r \delta_{x}^{2}\left[\varepsilon a_{s} \operatorname{sen}\left(\frac{s \pi j}{J}\right)+\varepsilon^{3} \chi_{j}\right]+\left(\lambda_{s}+\lambda_{0} \varepsilon^{2}\right) h\left\{\varepsilon a_{s} \operatorname{sen}\left(\frac{s \pi j}{J}\right)+\varepsilon^{3} \chi_{j}+\right. \\
\left.\frac{f^{\prime \prime \prime}(0)}{6}\left[\varepsilon a_{s} \operatorname{sen}\left(\frac{s \pi j}{J}\right)+\varepsilon^{3} \chi_{j}\right]^{3}+\ldots\right\}=0 .
\end{array}
$$

Considerando os termos de $\mathcal{O}\left(\varepsilon^{3}\right)$ obtemos :

$$
r \delta_{x}^{2} \chi_{j}+\lambda_{s} h \chi_{j}=-\lambda_{0} h a_{s} \operatorname{sen}\left(\frac{s \pi j}{J}\right)-\frac{f^{\prime \prime \prime}(0)}{6} \lambda_{s} h a_{s}{ }^{3} \operatorname{sen}^{3}\left(\frac{s \pi j}{J}\right) .
$$


Como no caso contínuo, a versão homogênea do problema acima é singular. Então para que exista solução, esta deve satisfazer a Alternativa de Fredholm, logo temos que:

$$
-\lambda_{0} h a_{s} \sum_{j=0}^{J} \operatorname{sen}^{2}\left(\frac{s \pi j}{J}\right)-\frac{f^{\prime \prime \prime}(0)}{6} \lambda_{s} h a_{s}{ }^{3} \sum_{j=0}^{J} \operatorname{sen}^{4}\left(\frac{s \pi j}{J}\right)=0
$$

Com isto temos que $a_{s}$ satisfaz:

$$
\left\{\begin{array}{l}
a_{s}=0 \\
\text { ou } \\
a_{s}{ }^{2}=\frac{-6 \lambda_{0} \sum_{j=0}^{J} \operatorname{sen}^{2}\left(\frac{s \pi j}{J}\right)}{f^{\prime \prime \prime}(0) \lambda_{s} \sum_{j=0}^{J} \operatorname{sen}^{4}\left(\frac{s \pi j}{J}\right)}
\end{array}\right.
$$

onde $\lambda_{s}$ é dado por (4.8) para $s=1,2, \ldots, J-1$. Estes valores aproximam bem a amplitude das soluções que bifurcam da solução trivial em $(P)$ ( ver 3.10 ), apenas quando $\lambda_{s}$ são boas aproximações dos autovalores de $(P)$.

A deficiência de $(P D)$ em determinar todos os pontos de bifurcação de $(P)$ e a amplitude das soluções bifurcantes, é muito importante pela seguinte razão. A dinâmica de $(P)$ é determinada pelo conjunto de soluções estáticas. Em qualquer cálculo numérico existe um limite inferior para $k\left(k_{\min }\right)$ e úm limite superior correspondente $J_{\max }$. Se resolvemos $(P)$ aproximadamente usando $(P D)$, então para $J_{\max }$ podemos escolher um valor de $\lambda$ tal que o conjunto de soluções estáticas de $(P D)$ seja uma representação muito pobre das soluções em $(P)$. Conseqüentemente a dinâmica de $(P)$ e $(P D)$ para $\lambda$ suficientemente grande, diferem substancialmente. Estes resultados são reflexos da incapacidade do sistema discreto em representar as características do problema contínuo. As soluções estáticas que bifurcam através de $\lambda=(s \pi)^{2} \mathrm{em}(P)$ possuem $s-1$ raízes. Desta forma para um $J_{\max }$ podemos sempre escolher um $\lambda$ tal que a solução estática ramificante não possa ser resolvida exatamente na malha.

Quando a malha é refinada, $(P D)$ pode apresentar soluções estáticas que não correspondam a nenhuma solução estática em $(P)$ ( ver Bohl [1]). Este fenômeno é chamado de " bifurcação fantasma ". Existem dois caminhos de identificação dessas soluções espúrias:

(1) Examinar o diagrama de bifurcação para uma seqüência de malhas. Pois quando a malha é refinada, as soluções espúrias se deslocam para o infinito ou se aglutinam em um ponto de retorno.

(2) Soluções variando em escalas comparáveis de malha, são freqüentemente espúrias. 
A presença de soluções espúrias tem importantes efeitos na solução de $(P)$, pois estas podem afetar a dinâmica global do problema discreto. Um exemplo de como soluções estáticas espúrias afetam computacionalmente a dependência no tempo é descrito em Stuart [23].

Vamos considerar $\xi_{s}=-1$ onde bifurcam soluções periódicas de $u_{j}^{n} \equiv 0$ em $(P D)$. De (4.7) temos:

$$
r=\frac{2+\lambda h(1-2 \theta)}{4(1-2 \theta) \operatorname{sen}^{2}\left(\frac{s \pi}{2 J}\right)} ; s=1,2, \ldots, J-1 .
$$

Como $r\left(=h / k^{2}\right)$ é sempre positivo temos que:

(i) Quando $1 / 2<\theta \leq 1$ a equação (4.10) não determina pontos de bifurcação. Assumindo que $\lambda h$ seja pequeno o suficiente para que o numerador de (4.10) seja positivo;

(ii) Quando $\theta=1 / 2$ existem $J-1$ pontos de bifurcação para $r=\infty$;

(iii) Quando $0 \leq \theta<1 / 2$ existem $J-1$ pontos de bifurcação para $r$ finito definido por (4.10).

O menor valor de $r$ em (4.10) é determinado com $s=J-1$ e denotaremos por $r_{c}$. Este é o valor de $r$ para o qual a instabilidade numérica ocorre na equação linearizada ( ver (2.27) ).

\subsection{Análise Não Linear Fraca}

Aqui empregamos a noção de estabilidade absoluta dada na Definição 2.8.1. Em particular fixamos $k$ e conseqüentemente $J$. Assim empregamos as teorias da bifurcação e da estabilidade assumindo um problema de dimensão fixa. Descrevemos a aplicação da teoria da estabilidade não linear fraca de $(P D)$ no caso $\theta=0$ usando o método utilizado por Newell [15], podendo esta ser extendida para $0 \leq \theta<1 / 2$.

Da equação (4.8) com $s=1$ temos o menor valor crítico de $\lambda$ no qual bifurcam soluções estáticas de $u_{j}^{n} \equiv 0$ em $(P D)$, assim:

$$
\lambda_{c}=\frac{4}{k^{2}} \operatorname{sen}^{2}\left(\frac{\pi}{2 J}\right) .
$$


Sendo $h=r k^{2}$ e substituindo na equação (4.10) obtemos:

$$
r=\frac{2}{4 \operatorname{sen}^{2}\left(\frac{s \pi}{2 J}\right)-\lambda k^{2}}
$$

Assim o menor valor crítico de $r$ para o qual bifurcam soluções periódicas é obtido quando $s=J-1$, e:

$$
r_{c}=\frac{2}{4 \operatorname{sen}^{2}\left(\frac{(J-1) \pi}{2 J}\right)-\lambda k^{2}} .
$$

Denotando por $h_{c}$ o menor valor crítico para o qual (4.12) ocorre podemos escrever $r_{c}=h_{c} / k^{2}$.

Existem três possibilidade de domínio dos parâmetros nos quais podemos realizar a análise não linear fraca em $(P D)$ :

(a) $r \approx r_{c}, \lambda<\lambda_{c} \mathrm{e}\left(\lambda-\lambda_{c}\right)=\mathcal{O}(1)$

(b) $\lambda \approx \lambda_{c}, r<r_{c} \mathrm{e}\left(r-r_{c}\right)=\mathcal{O}(1)$

(c) $r \approx r_{c}, \lambda \approx \lambda_{c}$.

O caso (a) é essencialmente o considerado por Newell [15], onde ele considera perturbações iniciais proporcionais à componente mais instável $(\operatorname{sen}((J-1) \pi j / J))$. Stuart [22] segue o trabalho de Matkowsky [13] para o problema contínuo e considera perturbações iniciais arbitrárias. $\mathrm{O}$ caso (b) é a discretização da análise feita na seção 3.5 ( Weiss [24] ). O caso (c) descreve a interação da instabilidade numérica com a dinâmica da EDP ( Stuart [22]).

Vamos analisar o caso (a). Procuramos a solução de $(P D)$ para $r$ próximo do valor crítico definido em (4.12). Desde que estamos considerando um problema de dimensão fixa ( $k$ fixo), qualquer perturbação em $r$ necessariamente conduz a uma perturbação de $h$. Conseqüentemente escrevemos:

$$
r=r_{c}+r_{0} \varepsilon^{2} ; \quad h=h_{c}+r_{0} k^{2} \varepsilon^{2} .
$$

Como $\left(\lambda-\lambda_{c}\right)=\mathcal{O}(1)$ e supondo $\lambda<\lambda_{c}$, procuramos uma expansão para $u_{j}^{n}$ na forma:

$$
u_{j}^{n} \approx \sum_{m=1}^{\infty} v_{m}(n, j, \eta) \varepsilon^{m}
$$

onde $\eta$ é uma função de $n$ que varia muito pouco com $n$, e será tratada como variável independente. Escolhemos os dados iniciais com amplitude pequena da forma:

$$
u_{j}^{0}=\varepsilon g(j k)
$$


Suponhamos que $v_{1}(n, j, \eta)$ tem a forma da solução linearizada, isto é:

$$
v_{1}(n, j, \eta)=\sum_{s=1}^{J-1} \xi_{s}^{n} \operatorname{sen}\left(\frac{s \pi j}{J}\right) A_{s}(n ; \varepsilon)
$$

onde $A_{s}(n ; \varepsilon) \equiv a_{s}(\eta)$ é suposto depender fracamente de $n$, e os $\xi_{s}$ são definidos por (4.7) $\operatorname{com} \theta=0, r=r_{c}, h=h_{c}$ e $f^{\prime}(0)=1$. Portanto

$$
\xi_{s}=1-4 r_{c} \operatorname{sen}^{2}\left(\frac{s \pi}{2 J}\right)+\lambda h_{c} ; \quad s=1,2, \ldots, J-1 .
$$

Sendo $r_{c}$ determinado por $s=J-1$, então temos que $\xi_{J-1}=-1$ e $\left|\xi_{s}\right|<1$ para $s=1,2, \ldots, J-2$. Uma suposição muito importante feita por Newell [15] é que:

$$
A_{s}(n+1 ; \varepsilon)-A_{s}(n ; \varepsilon)=\varepsilon^{2}\left[a_{s}(\eta+1)-a_{s}(\eta)\right] .
$$

O significado preciso da variável $\eta$ não está claro como no caso contínuo. Desde que $(P D)$ é defindo numa malha discreta (representando intervalos de tempo discreto), então frações do passo-tempo não tem significado. Para o nossó propósito é suficiente considerar (4.16) como a equação para $a_{s}(\eta+1)$ e notar que os $a_{s}$ podem ser eliminados numa relação de recorrência final ( ver (4.23)). Substituindo (4.13) e (4.14) em $(P D) \operatorname{com} \theta=0$ temos:

$$
\begin{array}{r}
\sum_{m=1}^{\infty}\left[v_{m}(n+1, j, \eta)-v_{m}(n, j, \eta)\right] \varepsilon^{m}=\left(r_{c}+r_{0} \varepsilon^{2}\right) \delta_{x}^{2}\left[\sum_{m=1}^{\infty} v_{m}(n, j, \eta) \varepsilon^{m}\right]+ \\
\lambda\left(h_{c}+r_{0} k^{2} \varepsilon^{2}\right)\left\{\sum_{m=1}^{\infty} v_{m}(n, j, \eta) \varepsilon^{m}+\frac{f^{\prime \prime \prime}(0)}{6}\left[\sum_{m=1}^{\infty} v_{m}(n, j, \eta)\right]^{3}+\ldots\right\}(4 .
\end{array}
$$

Sendo $\Delta x_{s} y_{s}=x_{s+1} \Delta y_{s}+y_{s} \Delta x_{s}$ (ver Mickens [14] pag. 35), então de (4.15) e (4.16) temos:

$$
\begin{array}{r}
\Delta_{n} v_{1}(n, j, \eta)=\varepsilon^{2} \sum_{s=1}^{J-1} \xi_{s}{ }^{n+1} \operatorname{sen}\left(\frac{s \pi j}{J}\right)\left[a_{s}(\eta+1)-a_{s}(\eta)\right]+ \\
\sum_{s=1}^{J-1} \xi_{s}{ }^{n+1} \operatorname{sen}\left(\frac{s \pi j}{J}\right) a_{s}(\eta)-\sum_{s=1}^{J-1} \xi_{s}{ }^{n} \operatorname{sen}\left(\frac{s \pi j}{J}\right) a_{s}(\eta)
\end{array}
$$

Substituindo (4.18) em (4.17) e igualando termo a termo a série de potências de $\varepsilon$ obtemos:

$\mathcal{L}_{d} v_{1}=0$

$\mathcal{L}_{d} v_{2}=0$ 


$$
\begin{aligned}
\mathcal{L}_{d} v_{3}= & \sum_{s=1}^{J-1}\left[\xi_{s}{ }^{n+1}\left(a_{s}(\eta)-a_{s}(\eta+1)\right) \operatorname{sen}\left(\frac{s \pi j}{J}\right)+\xi_{s}{ }^{n} r_{0} \delta_{x}{ }^{2} \operatorname{sen}\left(\frac{s \pi j}{J}\right) a_{s}(\eta)\right]+ \\
& \lambda r_{0} k^{2}\left[\sum_{s=1}^{J-1} \xi_{s}{ }^{n} \operatorname{sen}\left(\frac{s \pi j}{J}\right) a_{s}(\eta)\right]+ \\
& \lambda h_{c} \frac{f^{\prime \prime \prime}(0)}{6}\left[\sum_{s=1}^{J-1} \xi_{s}{ }^{n} \operatorname{sen}\left(\frac{s \pi j}{J}\right) a_{s}(\eta)\right]^{3}
\end{aligned}
$$

com $\left(v_{m}\right)_{0}=\left(v_{m}\right)_{J}=0$ onde $\mathcal{L}_{d} v_{j}^{n}=v_{j}^{n+1}-v_{j}^{n}-r_{c} \delta_{x}{ }^{2} v_{j}^{n}-\lambda h_{c} v_{j}^{n}$. Impondo as condições iniciais obtemos $\left(v_{1}\right)_{j}^{0}=g(j k)$ e $\left(v_{m}\right)_{j}^{0}=0$ para $m \geq 2$, assim $v_{2}$ é identicamente nula.

Como no caso contínuo termos seculares pode ocorrer, pois o operador diferença finita $\mathcal{L}_{d}$ ocorre em sucessivas ordens. Para anular os termos seculares. devemos remover os termos proporcionais a $(-1)^{n}$. Isto requer uma condição análoga a (3.19) para o caso discreto, ou seja:

$$
\lim _{N \rightarrow \infty} \frac{1}{N} \sum_{n=1}^{N} \sum_{j=0}^{J} \xi_{s}{ }^{n} \operatorname{sen}\left(\frac{s \pi j}{J}\right) r_{3}=0 ; \quad s=1,2, \ldots, J-1
$$

onde $r_{3}$ denota o lado direito de (4.21). Desde que $\left|\xi_{s}\right|<1$ para $s \neq J-1$, a única contribuição de (4.22) ocorre $\operatorname{com} s=J-1$. Note que:

$$
\delta_{x}^{2} \operatorname{sen}\left(\frac{s \pi j}{J}\right)=-4 \operatorname{sen}\left(\frac{s \pi j}{J}\right) \operatorname{sen}^{2}\left(\frac{s \pi}{2 J}\right)
$$

Desta forma com $s=J-1 \mathrm{em}(4.22)$ temos:

$$
a_{J-1}(\eta+1)-a_{J-1}(\eta)=\left[4 r_{0} \operatorname{sen}^{2}\left(\frac{(J-1) \pi}{2 J}\right)-\lambda r_{0} k^{2}\right] a_{J-1}(\eta)-b a_{J-1}{ }^{3}(\eta)
$$

onde $b$ é dado por:

$$
b=\frac{\lambda h_{c} f^{\prime \prime \prime}(0) \sum_{j=0}^{J} \operatorname{sen}^{4}\left(\frac{(J-1) \pi j}{J}\right)}{6 \sum_{j=0}^{J} \operatorname{sen}^{2}\left(\frac{(J-1) \pi j}{J}\right)}
$$

Da equação (4.12) temos que a igualdade acima pode ser escrita como:

$$
a_{J-1}(\eta+1)-a_{J-1}(\eta)=\frac{2 r_{0}}{r_{c}} a_{J-1}(\eta)-b a_{J-1}^{3}(\eta)
$$

A equação (4.23) descreve a evolução da amplitude de $a_{J-1}(\eta)$. As componentes associadas com outras amplitudes são decrescentes $\left(\left|\xi_{s}\right|<1\right)$. Desta forma (4.23) determina o comportamento assintótico de $(P D)$ para $\left|u_{j}^{n}\right| \ll 1$ e $r \approx r_{c}$. 
Por simplicidade denotaremos $a_{J-1}(\eta)=a(\eta)$, então (4.23) pode ser escrita na seguinte forma:

$$
a(\eta+1)=G(a(\eta))=\left(\frac{2 r_{0}}{r_{c}}+1\right) a(\eta)-b a^{3}(\eta)
$$

Definição 4.4.1: Um ponto c é chamado de ponto fixo de uma equação diferença se $c=G(c)$.

Uma equação da forma (4.24) tende para um ponto fixo, ou tende para $\pm \infty$, quando $\eta \rightarrow \infty$. As condições de convergência para um ponto fixo são dadas no sequinte terema:

Teorema 4.4.1: Seja c um ponto fixo da equação (4.24). Fazendo $\eta \rightarrow \infty$ temos que:

(i ) Se $\left|G^{\prime}(c)\right|<1$, então $a(\eta)$ converge para c;

(ii) Se $\left|G^{\prime}(c)\right|=1$, então $a(\eta)$ converge condicionalmente para c;

(iii) Se $\left|G^{\prime}(c)\right|>1$, então $a(\eta)$ diverge de c;

(iv) $S e|a(\eta+1)-c|<|a(\eta)-c| \forall \eta$, então $a(\eta)$ converge para $c$

Prova: ver Mickens [14].

O comportamento de (4.23) depende dos sinais dos parâmetros $r_{0}$ e $b$, assim consideremos os seguintes casos.

Para $r_{0}<0$ e $b>0,(4.24)$ apresenta um ponto fixo dado por $c=0$, então para que (4.24) convirga para $c$ impomos a condição (i) do Teorema (4.4.1), ou seja:

$$
\left|G^{\prime}(0)\right|<1 \Rightarrow\left|\frac{2 r_{0}}{r_{c}}+1\right|<1 \Rightarrow-r_{c}<r_{0}<0
$$

Através da condição (iv) do Teorema (4.4.1) temos que a condição inicial $a(0)$ deve satisfazer:

$$
\begin{array}{r}
\left|\left(\frac{2 r_{0}}{r_{c}}+1\right) a(0)-b a(0)^{3}\right|<|a(0)| \Rightarrow \\
-2-\frac{2 r_{0}}{r_{c}}<-b a(0)^{2}<\frac{-2 r_{0}}{r_{c}} \Rightarrow \\
\frac{2 r_{0}}{b r_{c}}<a(0)^{2}<\frac{1}{b}\left(\frac{2 r_{0}}{r_{c}}+2\right)
\end{array}
$$


Como $2 r_{0} / r_{c}<0$ o lado direito da desigualdade (4.26) leva a:

$$
|a(0)|<\left[\frac{1}{b}\left(\frac{2 r_{0}}{r_{c}}+2\right)\right]^{1 / 2}=\gamma
$$

Logo $|a(\eta)| \rightarrow 0$ quando $\eta \rightarrow \infty$ se $a(0)$ satisfaz a condição acima. Quando $|a(0)|=\gamma$ temos que $\pm a(0)=G(\mp a(0))$. Estes pontos são conhecidos como pontos antípodas. Neste caso deduzimos através de (4.14) e (4.16) que:

$$
u_{j}^{n} \approx \varepsilon(-1)^{n} \operatorname{sen}\left(\frac{(J-1) \pi j}{J}\right) \gamma
$$

quando $\eta \rightarrow \infty$. Desta forma $u_{j}^{n}$ aproxima-se de soluções periódicas em $n$ ( ver Figura 4.2-a).

Para $r_{0}<0$ e $b<0$ os pontos fixos de (4.24) são dados por:

$$
\begin{gathered}
c=0 \\
c= \pm\left(2 r_{0} / b r_{c}\right)^{1 / 2} .
\end{gathered}
$$

Primeiramente vamos analizar a possibilidade de (4.24) convergir para $c=0$. Impondo a condição (i) do Teorema (4.4.1) temos que (4.25) deve ser satisfeita. Da equação (4.26) temos que a condição inicial deve satisfazer:

$$
|a(0)|<\left(\frac{2 r_{0}}{b r_{c}}\right)^{1 / 2}
$$

Assim $|a(\eta)| \rightarrow 0$ quando $\eta \rightarrow \infty$ se $a(0)$ satisfaz a condição acima.

Consideremos o caso em que $c$ é dado pór (4.28), logo:

$$
\left|G^{\prime}\left( \pm\left(\frac{2 r_{0}}{b r_{c}}\right)^{1 / 2}\right)\right|=\left|\frac{4 r_{0}}{r_{c}}+1\right|>1 \text {. }
$$

Assim temos que $a(\eta)$ diverge de $c$ dado por (4.28), quando $\eta \rightarrow \infty$ ( condição (iii) do Teorema (4.4.1)). Aqui $c$ é um ponto crítico não nulo de (4.24). Deste modo instabilidade de amplitude finita é possível no problema não linear para valores de $r<r_{c}$, onde $a_{J-1}(0)$ é suficientemente grande (ver Figura 4.2-b ) Para $r_{0}>0$ e $b<0,(4.24)$ possue um único ponto fixo dado por $c=0$ e:

$$
\left|G^{\prime}(0)\right|=\left|\frac{2 r_{0}}{r_{c}}+1\right|>1
$$

Desta forma, da condição (iii) do Teorema (4.4.1), temos que $|a(\eta)| \rightarrow \infty$ quando $\eta \rightarrow \infty$ (ver Figura 4.2-d) Esta é uma manifeștação natural da instabilidade 


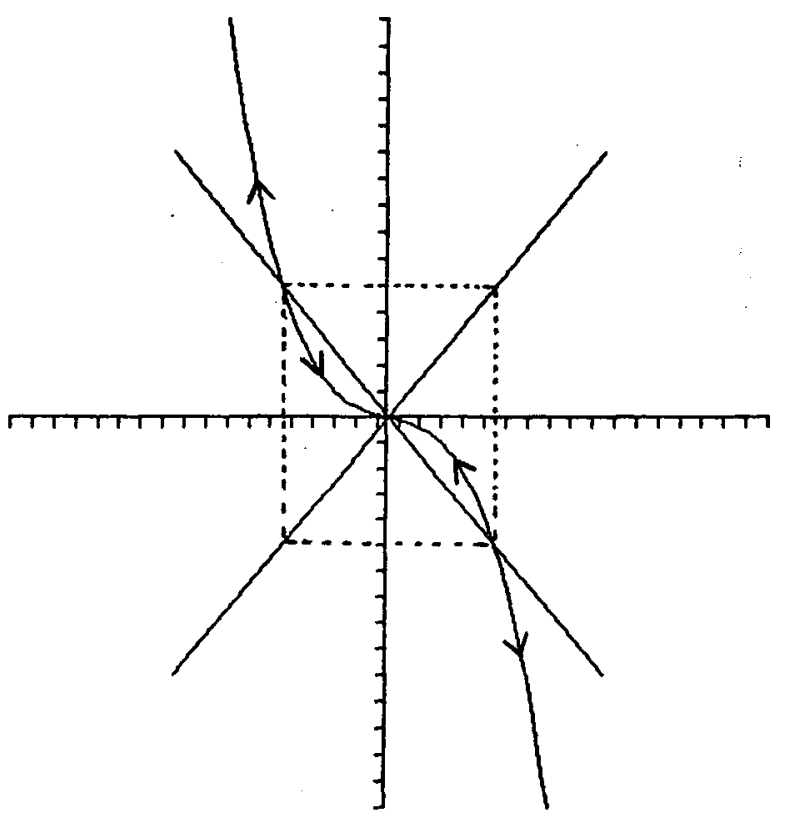

(a)

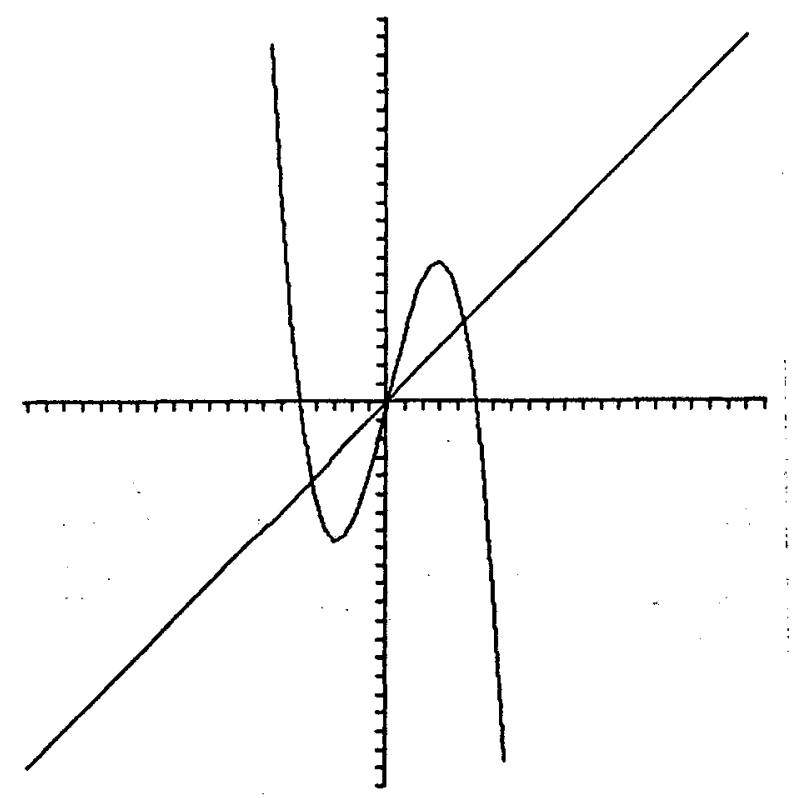

(c)

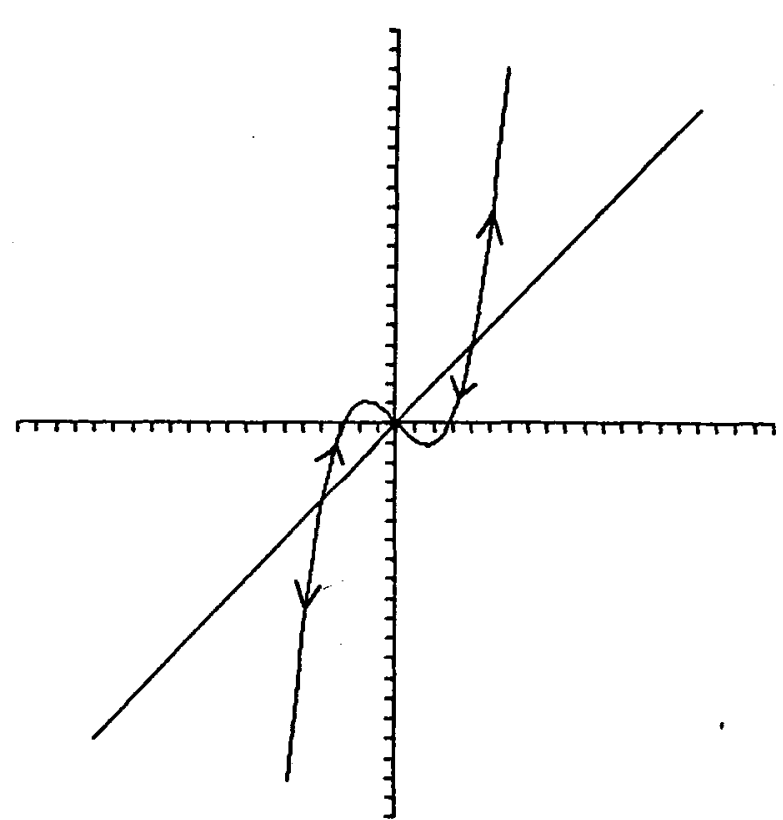

(b)

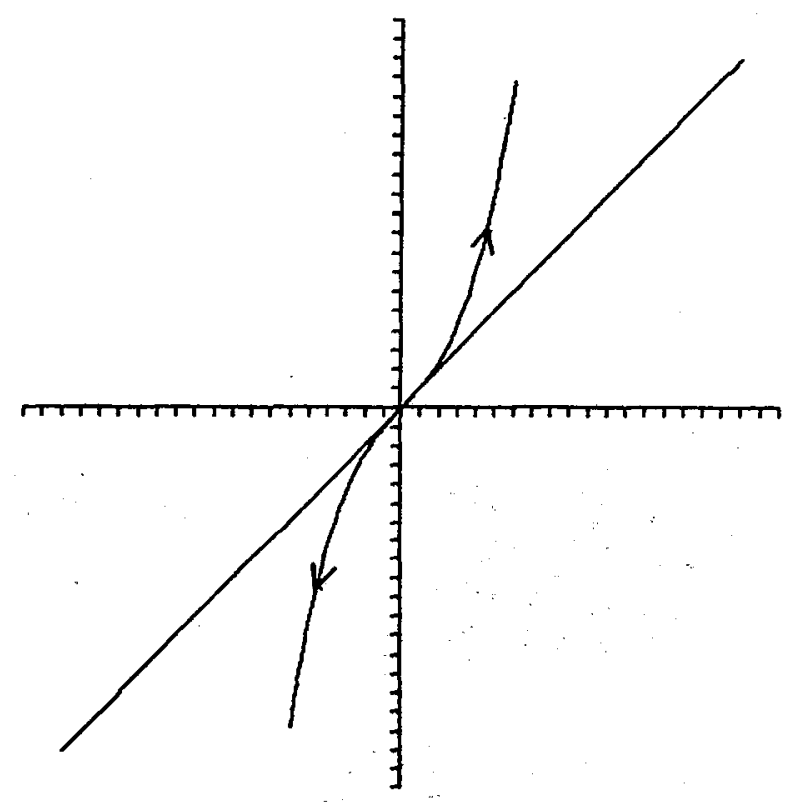

(d)

Figura 4.2: Curva de $a(\eta+1)=G(a(\eta))$ : (a) $r_{0}<0$ e $b>0$; (b) $r_{0}<0$ e $b<0$; (c) $r_{0}>0$ e $b>0$; (d) $r_{0}>0$ e $b<0$. As flechas indicam a direção das sucessivas iterações, exeto em (c). 


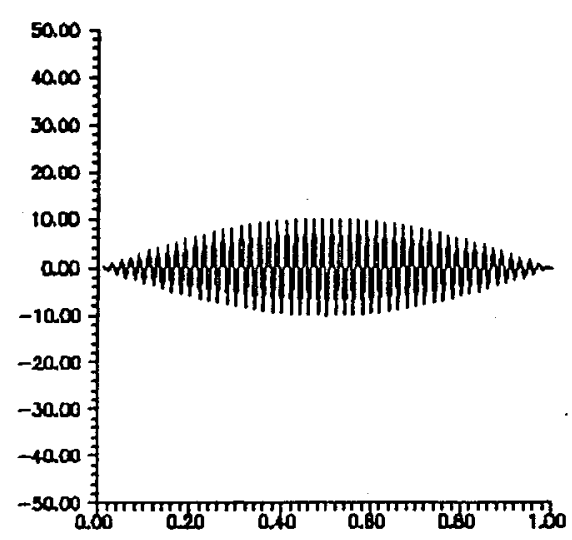

(a)

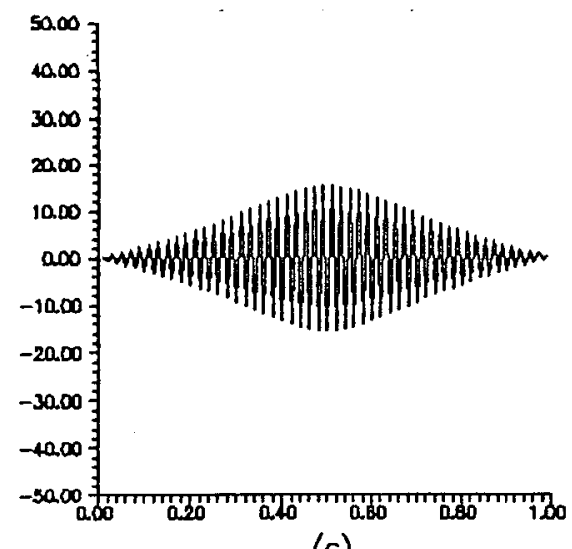

(c)

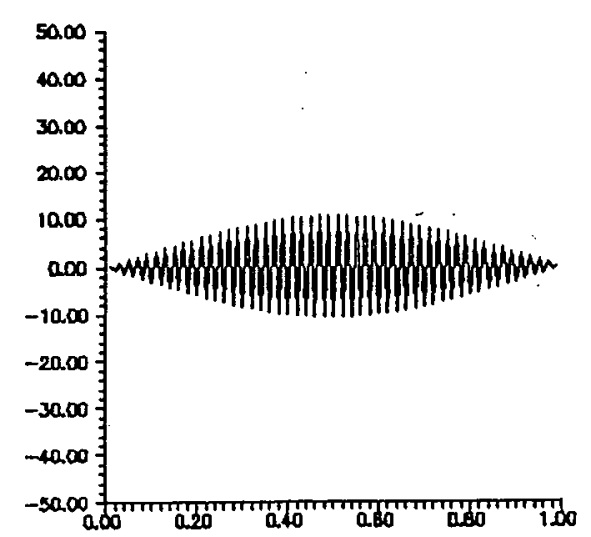

(b)

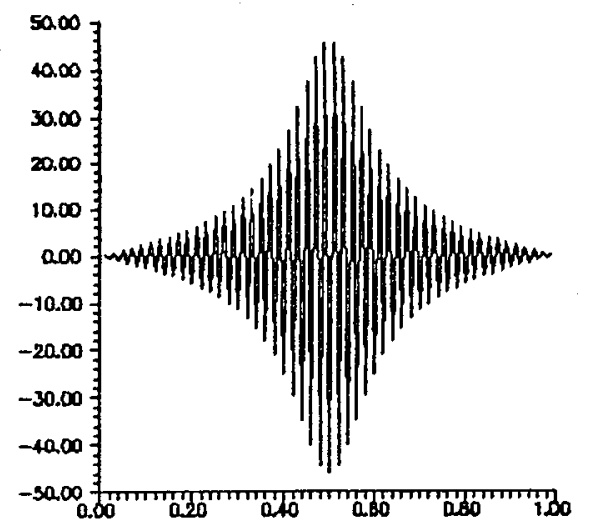

(d)

Figura 4.3: Solução de $(P D)$ para $r<r_{c} \operatorname{com} f^{\prime \prime \prime}(0)<0$ : (a) $t=0$; (b) $t=20 h$; (c) $t=100 h ;(d) t=150 h$.

Esta é uma manifestação natural da instabilidade numérica no problema parabólico, ou seja, o crescimento ilimitado do harmônico mais oscilatório. Note que a instabilidade é mais " forte" do que prediz a teoria linear $(b=0)$.

Para $r_{0}>0$ e $b>0,(4.24)$ possue os pontos fixos dados por (4.27) e (4.28). Quando $c=0$ temos que:

$$
\left|G^{\prime}(0)\right|=\left|\frac{2 r_{0}}{r_{c}}+1\right|>1
$$

Desta forma $|a(\eta)| \not 0$ quando $\eta \rightarrow \infty$. Considerando o caso em que $c$ é dado por (4.28) e impondo a condição (i) do Teorema (4.4.1) obtemos:

$$
\left|\frac{-4 r_{0}}{b r_{c}}+1\right|<1 \Rightarrow 0<r_{0}<\frac{r_{c}}{2}
$$

Com isto vemos que existe a possibilidade de que algurnas soluções convirgam para o ponto fixo (4.28), quando $\eta \rightarrow \infty$ (ver Figura 4.2-d). Os pontos críticos dados por (4.28) podem ser atratores. Algumas soluções de (4.24) são tais que $a(\eta) \rightarrow c$ quando $\eta \rightarrow \infty$. Consequëntemente de (4.14) e (4.16) conclui-se:

$$
u_{j}^{n}=\varepsilon(-1)^{n} \operatorname{sen}\left(\frac{(J-1) \pi j}{J}\right) c
$$



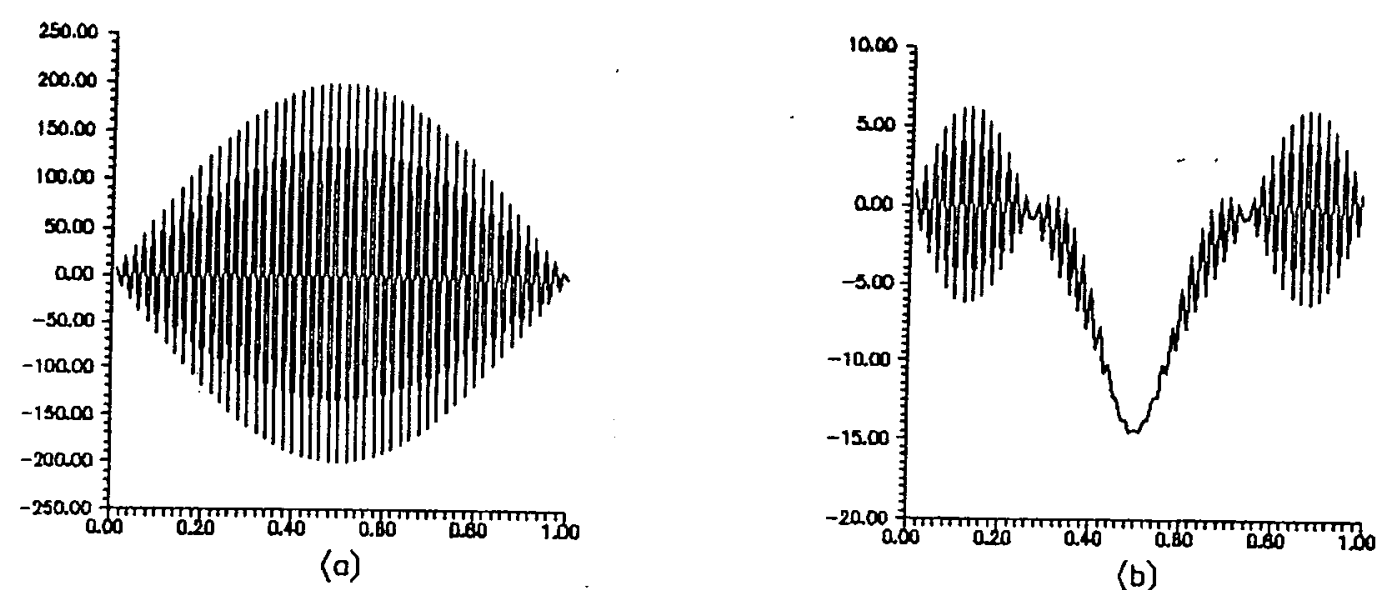

(b)

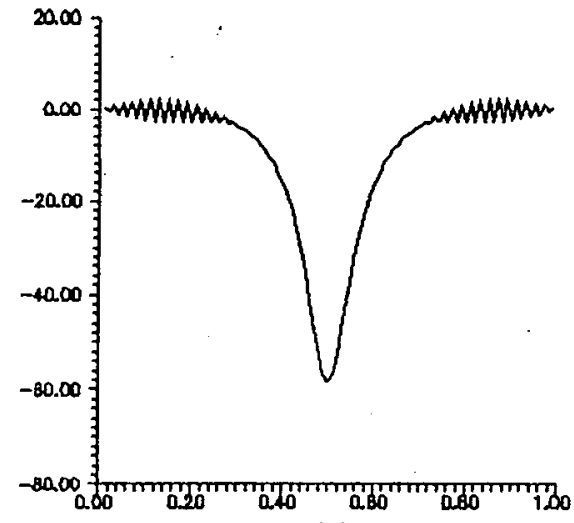

(c)

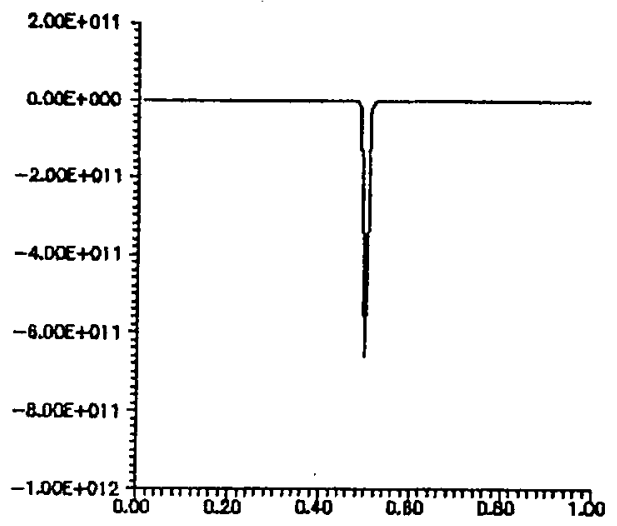

(d)

Figura 4.4: Solução de $(P D)$ para $r<r_{c} \operatorname{com} f^{\prime \prime \prime}(0)>0$ : (a) $t=0$; (b) $t=100 h$; (c) $t=190 h ;(\mathrm{d}) t=198 h$.

quando $\eta \rightarrow \infty$. Desta forma $u_{j}^{n}$ evolui na direção de soluções oscilatórias em $n$. Este estado oscilatório estável é puramente um produto da discretização. Este não é um comportamento padrão de instabilidade pois seu crescimento é limitado. Aqui a instabilidade numérica é inibida pela não-linearidade e os dois efeitos se balanceiam para produzir uma periodicidade.

Em resumo, no caso (a) notamos que a instabilidade numérica em $(P D)$ esta associada com a bifurcação de uma órbita periódica através da solução trivial $u_{j}^{n} \equiv 0$. A manifestação da instabilidade numérica depende crucialmente das propriedades destas órbitas periódicas. Se a órbita repele os dados iniciais numa vizinhança, então a instabilidade numérica ocorre de uma forma familiar (crescimento ilimitado de um harmônico oscilatório). Instabilidade numérica de amplitude finita também ocorre para valores de $r$ menores que os valores críticos prescritos através da teoria linear. Isto é demostrado na Figura 4.3 que mostra a solução de $(P D)$ com os seguintes parâmetros: $k=0.01, \lambda=1.0, r<r_{c}$ com $f(u)=u-u^{3}\left(r_{0}<0\right.$ e $\left.b<0\right)$. Os dados iniciais são proporcionais à componente mais instável $\left(u_{0}(x)=10 \operatorname{sen}(99 \pi x)\right)$. A evolução inicial da componente é governada por (4.23). Eventualmente outras componentes são estimuladas, uma ocorrência da não linearidade. Logo ápos na Figura 4.3-d a solução atinge 


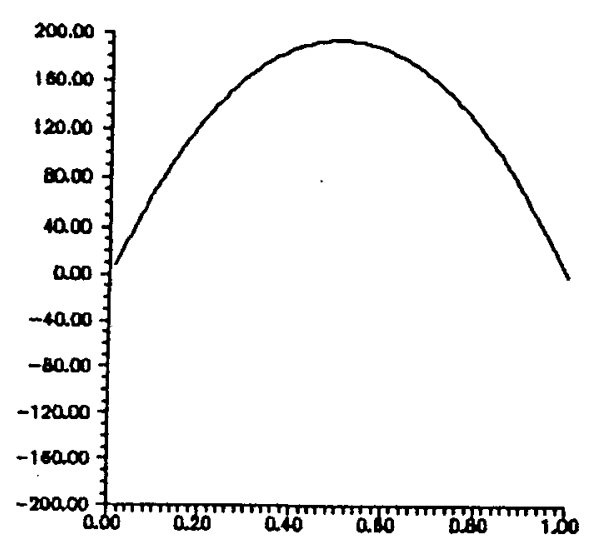

(o)

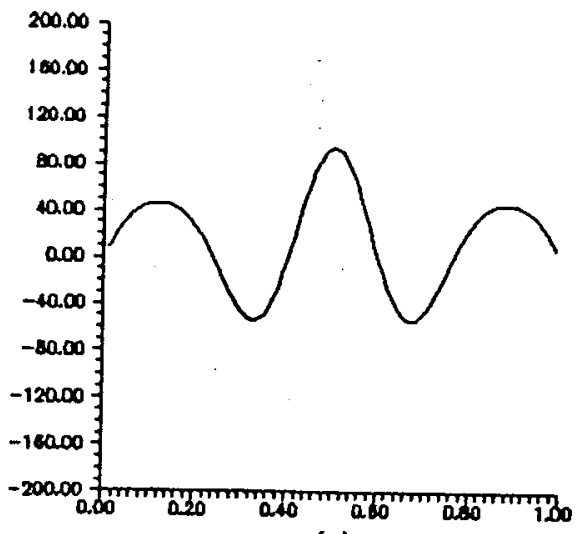

(c)

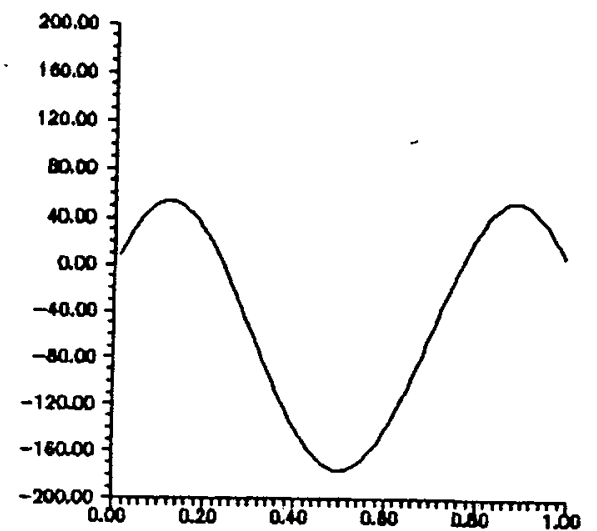

(b)

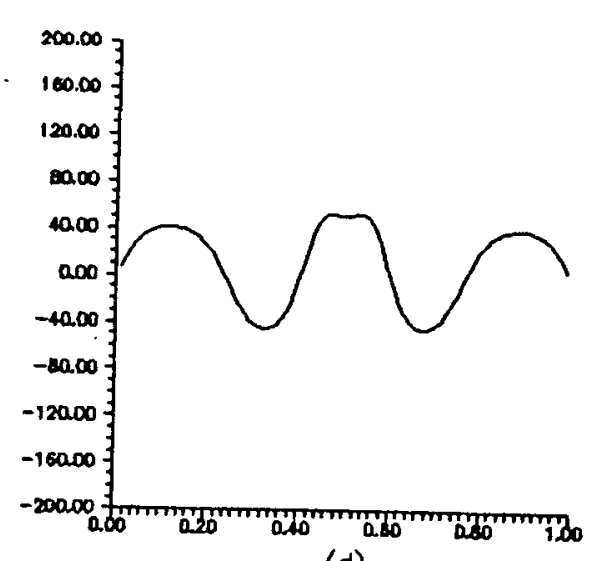

(d)

Figura 4.5: Solução de $(P D)$ para $r<r_{c} \operatorname{com} f^{\prime \prime \prime}(0)<0$ : (a) $t=0$; (b) $t=h$; (c) $t=2 h$; (d) $t=3 h$.

"overflow". Sendo $f(0)=0$ podemos mostrar que a norma $L_{2}$ da solução de $(P)$ é uma função monotonicamente decrescente, satisfazendo para $t>0$ :

$$
\|u(\cdot, t)\|<\|u(\cdot, 0)\| e^{-\beta t}
$$

onde $\beta>0$ ( ver Teorema 4.1 em Conway, Hoff e Smoller [4] )

A Figura 4.4 foi gerada com os mesmos parâmetros que os da Figura 4.3 onde $f(u)=u+u^{3}\left(r_{0}<0 \mathrm{e} b>0\right)$. Os dados iniciais são proporcionais à componente mais instável $\left(u_{0}(x)=199.85 \operatorname{sen}(99 \pi x)\right)$. Neste caso a Figura 4.4-d mostra o crescimento ilimitado dos pontos numa vizinhança de $x=0.5$.

Os parâmetros utilizados para gerar a Figura 4.5 são os mesmos da Figura 4.3, mas com os dados iniciais tendo um perfil parabólico $\left(u_{0}(x)=\right.$ $\left.780 *\left(-x^{2}+x\right)\right)$. Enquanto a solução assintótica está correta $\left(\left|u_{j}^{n}\right| \rightarrow 0\right.$ quando $n \rightarrow \infty)$, as soluções transitórias não estão, pois é possivel mostrar através do Teorema da Comparação (ver Smoller [20] pag. 94), que $u(x, t) \geq 0, \forall t$. A estrutura espacial mostrada na Figura 4.5-c é caracteristica de instabilidade numérica subcrítica, e esta pode ser explicada através de uma análise da equação modificada (ver Stuart [23]).

Se a órbita periódica é um atrator para os dados iniciais numa vizi- 
nhança, então a instabilidade numérica do método é mais sutil, visto que esta manifestação está limitada pelo comportamento periódico em $n$. Isto é observado na Figura 4.6 que mostra a solução de $(P D) \operatorname{com} f(u)=u+u^{3}, k=0.05, \lambda=1$ e $r>r_{\mathrm{c}}\left(r_{0}>0\right.$ e $\left.b>0\right)$. Os dados iniciais tem um perfil parabólico de amplitude pequena $\left(u_{0}(x)=5 *\left(-x^{2}+x\right)\right)$. Para $n$ grande as soluções oscilam entre o perfil mostrado na Figura 4.6-d e sua oposta em sucessivos passos de tempo. A Figura 4.6-e mostra o gráfico do comportamento da norma $L_{2}$ da solução em comparação ao tempo. Inicialmente a amplitude decai, enquanto a instabilidade linear amplifica significativamente a componente mais instável. Depois disso a amplitude continua a crescer enquanto a não linearidade balanceia a instabilidade linear e um equilibrio é atingido.

Consideremos o caso (b). Neste caso analisaremos as soluções de (PD) para

$$
\lambda=\lambda_{c}+\lambda_{0} \varepsilon^{2},
$$

supondo que $r-r_{c}=\mathcal{O}(1)$ e $r<r_{c}$. A análise é análoga à considerada no caso (a). Desejamos uma expansão para $u_{j}^{n}$ da forma (4.14), onde $v_{1}(n, j, \eta)$ é dada por (4.15). Os $\xi_{s}$ são definidos por (4.7), $\operatorname{com} \theta=0$ e $\lambda=\lambda_{c}(\operatorname{ver}(4.11)) \mathrm{e}$ satisfazem:

$$
\begin{aligned}
& \xi_{s}=1-4 r \operatorname{sen}^{2}\left(\frac{s \pi}{2 J}\right)+\lambda_{c} h \\
& =1-4 r \operatorname{sen}^{2}\left(\frac{s \pi}{2 J}\right)+4 r \operatorname{sen}^{2}\left(\frac{\pi}{2 J}\right)
\end{aligned}
$$

Desta forma $\xi_{1}=1 \mathrm{e}\left|\xi_{s}\right|<1$ para $s=2,3, \ldots, J-1$. Substituindo $u_{j}^{n}$ dado por (4.14) e $\lambda$ dado por (4.29) em (PD), e igualando termo a termo a série de potências de $\varepsilon$ obtemos:

$$
\begin{aligned}
\mathcal{L}_{d} v_{1}= & 0 \\
\mathcal{L}_{d} v_{2}= & 0 \\
\mathcal{L}_{d} v_{3}= & \sum_{s=1}^{J-1} \xi_{s}{ }^{n+1} \operatorname{sen}\left(\frac{s \pi j}{J}\right)\left(a_{s}(\eta)-a_{s}(\eta+1)\right)+\lambda_{0} h \sum_{s=1}^{J-1} \xi_{s}{ }^{n} \operatorname{sen}\left(\frac{s \pi j}{J}\right) a_{s}(\eta) \\
& +\lambda_{c} h \frac{f^{\prime \prime \prime}(0)}{6}\left[\sum_{s=1}^{J-1} \xi_{s}{ }^{n} \operatorname{sen}\left(\frac{s \pi j}{J}\right) a_{s}(\eta)\right]^{3}
\end{aligned}
$$

onde $\mathcal{L}_{d} v_{j}^{n}=v_{j}^{n+1}-v_{j}^{n}-r \delta_{x}{ }^{2} v_{j}^{n}-\lambda_{c} h v_{j}^{n}$. Impondo as condições iniciais temos que $\left(v_{1}\right)_{j}^{0}=g(j k) \mathrm{e}\left(v_{2}\right)_{j}^{0}=\left(v_{3}\right)_{j}^{0}=0$. Como no caso anterior, para que não ocorram termos seculares devemos impor a condição (4.22). Onde $r_{3}$ denota o lado direito de (4.32). Desde que $\left|\xi_{s}\right|<1$ para $s \neq 1$ obtemos:

$$
a_{1}(\eta+1)-a_{1}(\eta)=\lambda_{0} h a_{1}(\eta)+b a_{1}^{3}(\eta)
$$

onde :

$$
b=\frac{\lambda_{c} h f^{\prime \prime \prime}(0) \sum_{s=1}^{J-1} \operatorname{sen}^{4}\left(\frac{\pi j}{J}\right)}{6 \sum_{s=1}^{J-1} \operatorname{sen}^{2}\left(\frac{\pi j}{J}\right)} .
$$




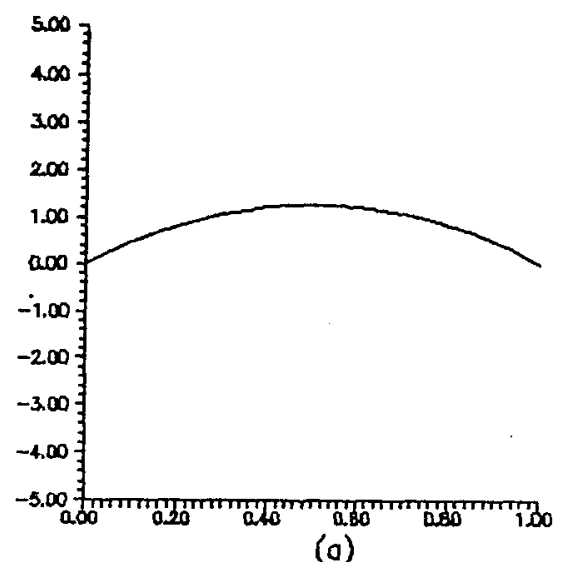

(a)

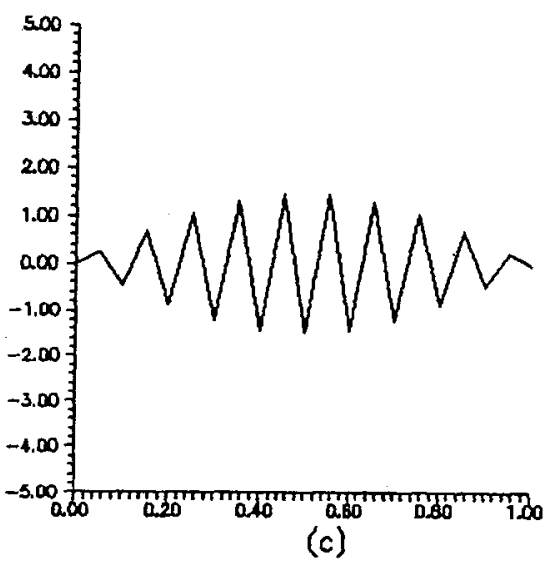

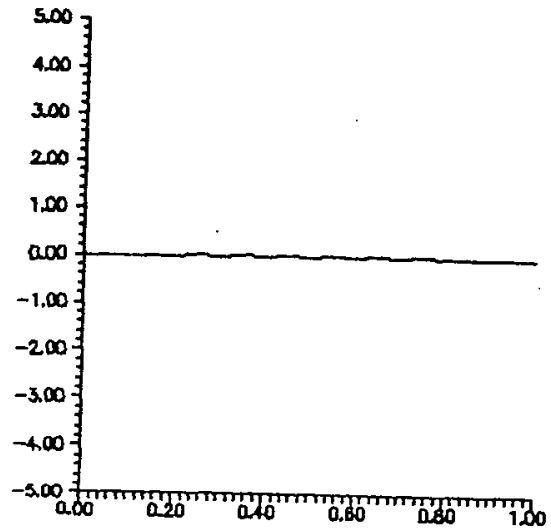

(b)

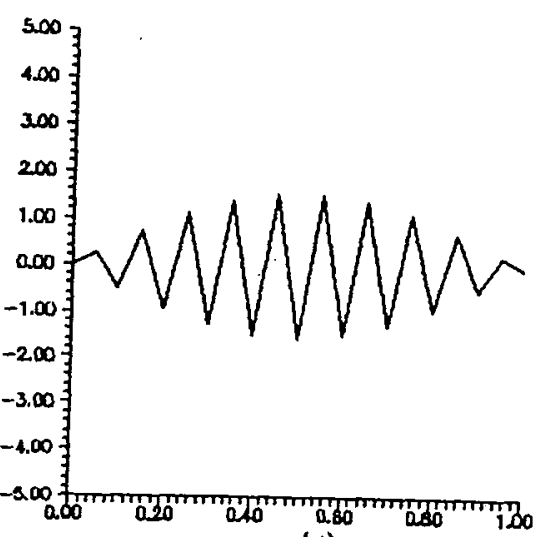

(d)

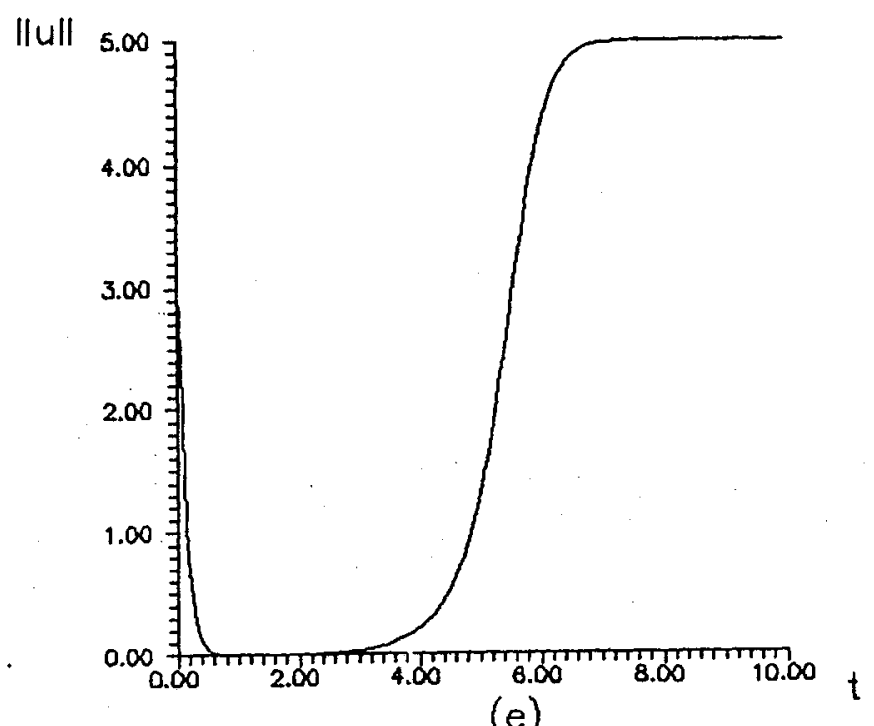

(e)

Figura 4.6: Solução de $(P D)$ para $r>r_{c} \operatorname{com} f^{\prime \prime \prime}(0)>0$ : (a) $t=0$; (b) $t=1500 h$; (c) $t=5000 h ;$ (d) $t=7500 h ;$ (e) Comportamento da norma $L_{2}$ em relação ao tempo. 


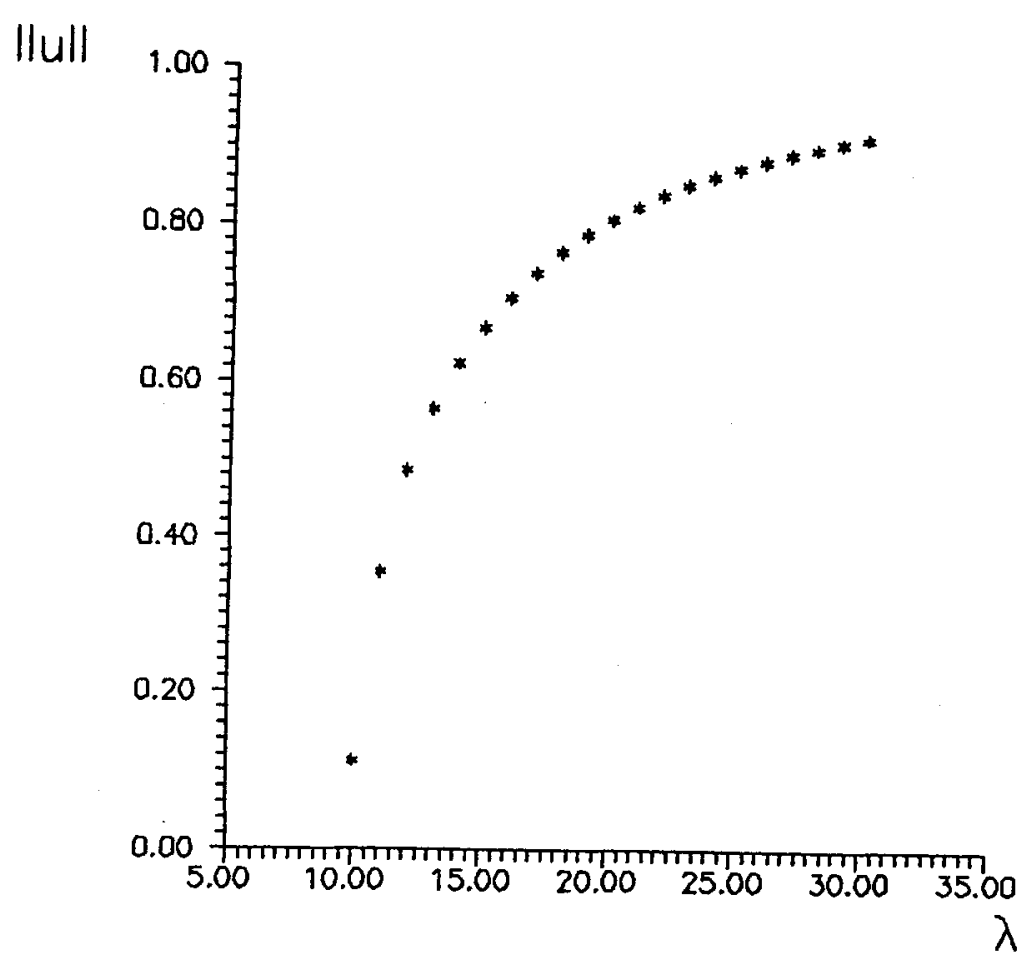

Figura 4.7: Diagrama de Bifurcação de $(P D) \operatorname{com} \lambda>\lambda_{c}$ e $t=2000 h$

Reescrevendo (4.33) em termos de $A_{1}(n ; \varepsilon)$, e usando (4.16) obtemos:

$$
\frac{A_{1}(n+1 ; \varepsilon)-A_{1}(n ; \varepsilon)}{\varepsilon^{2} h}=\lambda_{0} A_{1}(n ; \varepsilon)+\frac{b}{h} A_{1}{ }^{3}(n ; \varepsilon) .
$$

Tomando o limite quando $k, h \rightarrow 0$ recuperamos (3.20), e a solução de (4.33) é essencialmente igual ao caso contínuo, resumido nas Figuras (3.5) e (3.6).

A Figura (4.7) foi gerada com $k=0.05, r<r_{c}$, e $f(u)=u-u^{3}$. Os dados iniciais são proporcionais à componente $\operatorname{sen}(\pi x)$ com amplitude bem pequena. Aqui a norma da solução é calculada com $n$ grande e fixo $(n=2000)$ e $\lambda>\lambda_{c}$. O comportamento da norma da solução de $(P D)$ é similar a $(P)$ numa vizinhança do ponto $\lambda \approx \pi^{2}$ ( comparar Figura 4.7 com Figura 3.6 ). Esta análise é válida para outros pontos onde bifurcam soluções estáticas, exceto nos casos onde os pontos de bifurcação no problema $(P D)$ e as correspondentes autofunções não são bem representadas com o crescimento de $s$.

Analisaremos o caso (c), onde $\lambda$ e $r$ estão próximos de seus valores críticos $\lambda_{c}$ e $r_{c}$. Stuart [22] usa uma extensão do método empregado por Newell [15] no caso (a). O autovalor do problema linearizado (4.3), que determina as bifurcações é de multiplicidade 2. A existência de um autovalor duplo quase sempre está associado com bifurcação secundária, é o que ocorre neste caso. Mostraremos que o início da instabilidade numérica no problema não-linear $(P D)$, não 
está associada apenas com a bifurcação periódica da solução trivial (caso (a)) mas também com a bifurcação de órbitas periódicas de ramos estáticos não triviais. Assumimos como em (4.14)-(4.16) uma expansão para $u_{j}^{n}$ e que:

$$
\left\{\begin{array}{l}
\lambda=\lambda_{c}+\lambda_{0} \varepsilon^{2} \\
h=h_{c}+r_{0} k^{2} \varepsilon^{2} \\
r=r_{c}+r_{0} \varepsilon^{2}
\end{array}\right.
$$

Da equação (4.7) $\operatorname{com} \theta=0$ temos que:

$$
\xi_{s}=1-4 r_{c} \operatorname{sen}\left(\frac{s \pi}{2 J}\right)+\lambda_{c} h_{c}
$$

que satisfaz $\xi_{1}=1, \quad \xi_{J-1}=-1$ e $\left|\xi_{s}\right|<1$ para $s=2,3, \ldots, J-2$. Aqui $r_{c}$ é dado por (4.12) $\operatorname{com} \lambda=\lambda_{c}$. Substituindo (4.14)-(4.16) e (4.34) em (PD) e igualando. as sucessivas potências de $\varepsilon$ temos:

$$
\begin{aligned}
\mathcal{L}_{d} v_{1}= & 0 \\
\mathcal{L}_{d} v_{2}= & 0 \\
\mathcal{L}_{d} v_{3}= & \sum_{s=1}^{J-1}\left[\xi_{s}{ }^{n+1}\left(a_{s}(\eta)-a_{s}(\eta+1)\right) \operatorname{sen}\left(\frac{s \pi j}{J}\right)\right]+r_{0} \delta_{x}{ }^{2}\left[\sum_{s=1}^{J-1} \xi_{s}{ }^{n} \operatorname{sen}\left(\frac{s \pi j}{J}\right) a_{s}(\eta)\right]+ \\
& \left(\lambda_{0} h_{c}+r_{0} k^{2} \lambda_{c}\right)\left[\sum_{s=1}^{J-1} \xi_{s}{ }^{n} \operatorname{sen}\left(\frac{s \pi j}{J}\right) a_{s}(\eta)\right]+ \\
& \lambda_{c} h_{c} \frac{f^{\prime \prime \prime}(0)}{6}\left[\sum_{s=1}^{J-1} \xi_{s}{ }^{n} \operatorname{sen}\left(\frac{s \pi j}{J}\right) a_{s}(\eta)\right]^{3}
\end{aligned}
$$

onde $\mathcal{L}_{d} v_{j}^{n}=v_{j}^{n+1}-v_{j}^{n}-r_{c} \delta_{x}^{2} v_{j}^{n}-\lambda_{c} h_{c} v_{j}^{n}$. Resolvemos (4.37) usando a decomposição de Fourier em termos das autofunções $\operatorname{sen}(s \pi j / J)$ e escolhemos os $a_{s}(\eta)$ de maneira a suprimir a ocorrência dos termos seculares em $n$. Desta forma, na decomposição de $v_{3}$ que corresponde a $s=1$, removemos os termos constantes em $n$, e na decomposição que corresponde a $s=J-1$, removemos os termos proporcionais a $(-1)^{n}$. Impondo a condição dada em (4.22), onde $r_{3}$ denota $o$ lado direito de (4.37), obtemos as seguintes relações de recorrência:

$$
\begin{aligned}
a_{1}(\eta+1)-a_{1}(\eta) & =d_{1} a_{1}(\eta)+b_{1} \dot{a}_{1}^{3}(\eta)+b_{2} a_{1}(\eta) a_{J-1}{ }^{2}(\eta) \\
a_{J-1}(\eta+1)-a_{J-1}(\eta) & =d_{2} a_{J-1}(\eta)+b_{3} a_{J-1}{ }^{3}(\eta)+b_{4} a_{J-1}(\eta) a_{1}{ }^{2}(\eta)
\end{aligned}
$$

onde os coeficientes são dados por:

$$
\begin{aligned}
& d_{1}=\lambda_{c} r_{0} k^{2}+\lambda_{0} h_{c}-4 r_{0} \operatorname{sen}^{2}\left(\frac{\pi}{2 J}\right)=\lambda_{0} h_{c}, \\
& d_{2}=4 r_{0} \operatorname{sen}^{2}\left(\frac{(J-1) \pi}{2 J}\right)-\lambda_{0} h_{c}-\lambda_{c} r_{0} k^{2}=\frac{2 r_{0}}{r_{c}}-\lambda_{0} h_{c},
\end{aligned}
$$




$$
\begin{aligned}
& b_{1}=\lambda_{c} h_{c} f^{\prime \prime \prime}(0) \frac{\sum_{j=1}^{J-1} \operatorname{sen}^{4}\left(\frac{\pi j}{J}\right)}{6 \sum_{j=1}^{J-1} \operatorname{sen}^{2}\left(\frac{\pi j}{J}\right)}, \\
& b_{2}=\lambda_{c} h_{c} f^{\prime \prime \prime}(0) \frac{\sum_{j=1}^{J-1} \operatorname{sen}^{2}\left(\frac{\pi j}{J}\right) \operatorname{sen}^{2}\left(\frac{(J-1) \pi j}{J}\right)}{2 \sum_{j=1}^{J-1} \operatorname{sen}^{2}\left(\frac{\pi j}{J}\right)}, \\
& b_{3}=-\lambda_{c} h_{c} f^{\prime \prime \prime}(0) \frac{\sum_{j=1}^{J-1} \operatorname{sen}^{4}\left(\frac{(J-1) \pi j}{J}\right)}{6 \sum_{j=1}^{J-1} \operatorname{sen}^{2}\left(\frac{(J-1) \pi j}{J}\right)}, \\
& b_{4}=-\lambda_{c} h_{c} f^{\prime \prime \prime}(0) \frac{\sum_{j=1}^{J-1} \operatorname{sen}^{2}\left(\frac{\pi j}{J}\right) \operatorname{sen}^{2}\left(\frac{(J-1) \pi j}{J}\right)}{2 \sum_{j=1}^{J-1} \operatorname{sen}^{2}\left(\frac{(J-1) \pi j}{J}\right)}
\end{aligned}
$$

Desde que $\left|\xi_{s}\right|<1, s=2,3, \ldots, J-2, \quad \xi_{1}=1$ e $\xi_{J-1}=-1$ podemos deduzir através de (4.14) e (4.15) que, para $n$ grande, $(P D)$ tem uma solução aproximada dada por:

$$
u_{j}^{n} \approx \varepsilon v_{1}(n, j, \eta) \approx \varepsilon a_{1}(\eta) \operatorname{sen}\left(\frac{\pi j}{J}\right)+\varepsilon(-1)^{n} a_{J-1}(\eta) \operatorname{sen}\left(\frac{(J-1) \pi j}{J}\right) .
$$

Assim a evolução de $u_{j}^{n}$ é controlada por $a_{1}(\eta)$ e $a_{J-1}(\eta)$ descritos em (4.38) e (4.39).

As equações (4.38) e (4.39) possuem quatro pontos críticos, a saber:

$$
(0,0) ;\left(0, \sqrt{\frac{-d_{2}}{b_{3}}}\right) ;\left(\sqrt{\frac{-d_{1}}{b_{1}}}, 0\right) ;\left(\sqrt{\frac{d_{2} b_{2}-b_{1} b_{3}}{b_{1} b_{3}-b_{2} b_{4}}} ; \sqrt{\frac{d_{1} b_{4}-d_{2} b_{1}}{b_{1} b_{3}-b_{2} b_{4}}}\right) .
$$

0 segundo e terceiro pontos críticos ocorrem quando uma bifurcação origina-se do primeiro ponto crítico ( trivial ). O quarto ocorre quando uma bifurcação origina-se do segundo ou terceiro ponto crítico. Em termos da solução de $(P D)$, o segundo ponto critico representa uma solução oscilante em $n$, o terceiro uma solução estacionária e o quarto uma mistura dos dois. A existência de uma componente mista é importante visto que esta demonstra que o início da instabilidade em $(P D)$ está associada com a bifurcação de orbitas periódicas (em $n$ ) através da solução não trivial.

É certo que nossa análise é restrita para uma vizinhança de $\lambda \approx \lambda_{c} \mathrm{e}$ $r \approx r_{c}$, mas através da continuação para outros valores dos parâmetros, deduzimos que a bifurcação secundária de órbitas periódicas, proveniente de soluções estáticas é uma ocorrência genérica em $(P D)$. O número de possíveis diagramas de bifurcação para (4.38) e (4.39) é grande devido ao número de parâmetros. Descrevemos dois mostrando a bifurcação secundária da componente mista, proveniente do estado estacionário ( Figura 4.8-a) e a bifurcação proveniente do 


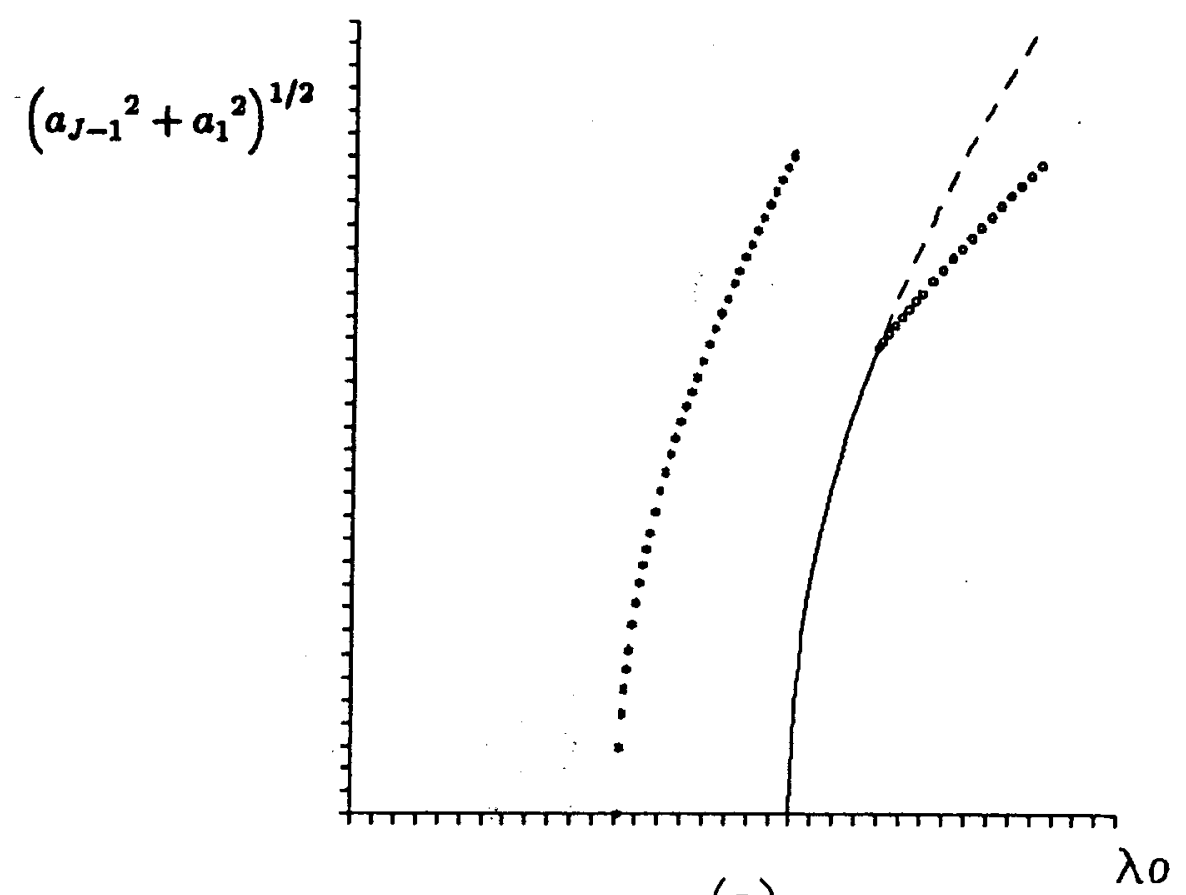

(a)

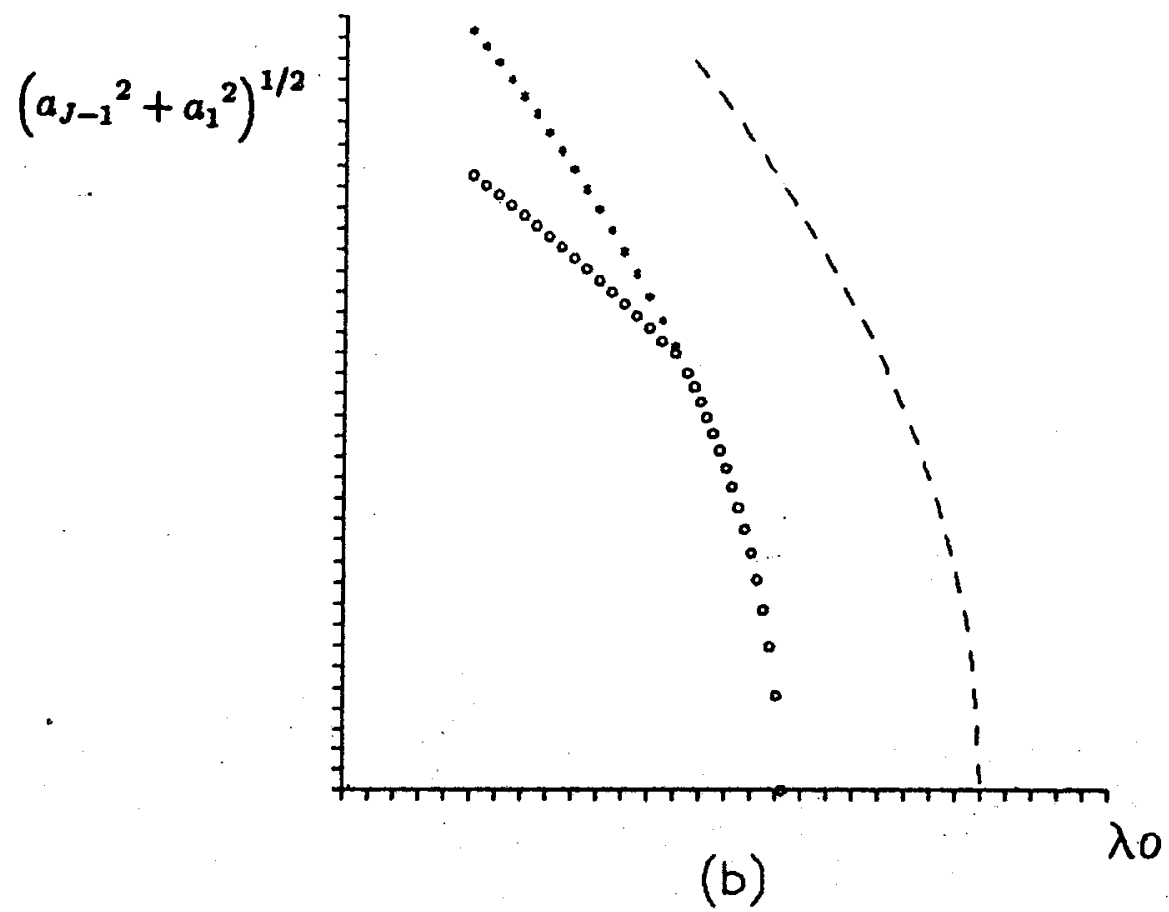

Figura 4.8: Diagrama de bifurcação: (a) estado estacionário $\left(f^{\prime \prime \prime}(0)<0\right.$ e $\left.r_{0}<0\right)$; (b)estado oscilatório $\left(f^{\prime \prime \prime}(0)>0\right.$ e $\left.r_{0}<0\right)$. Legenda : $* * *$ solução periódica instável; - solução estática estável; - - - solução estática instável; 000 solução periódica estável. 


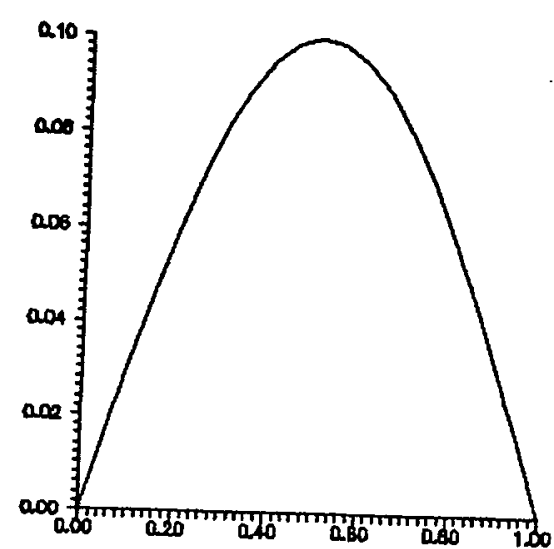

(a)

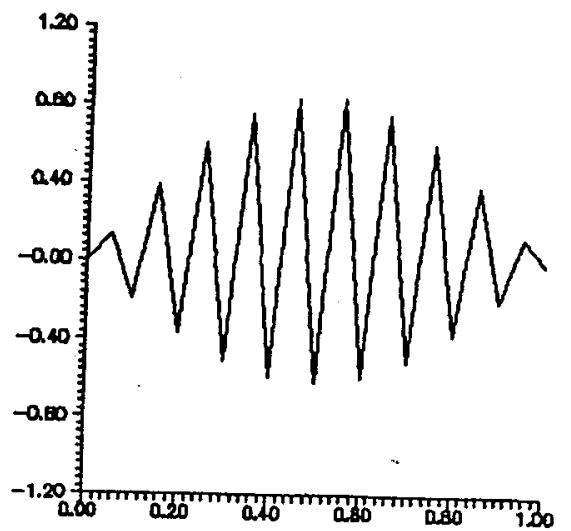

(c)

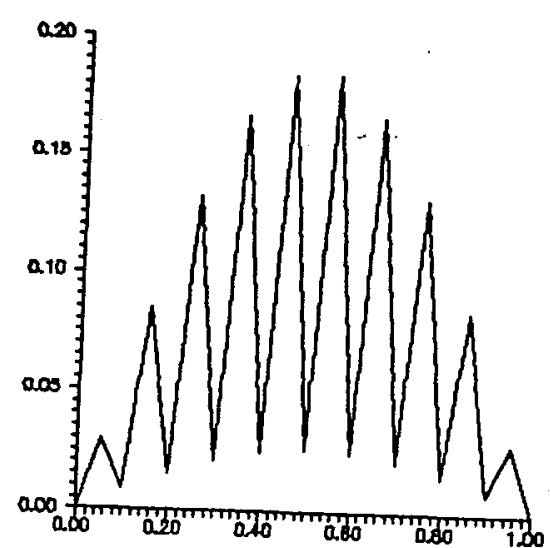

(b)

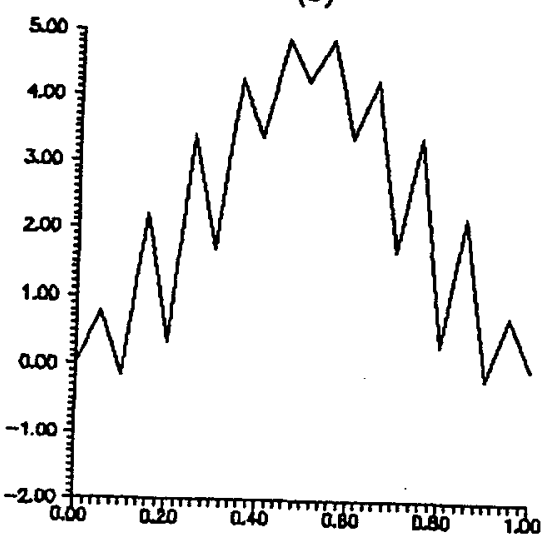

(d)

Figura 4.9: Solução de $(P D)$ para $r>r_{c}, \lambda>\lambda_{c}$. e $f^{\prime \prime \prime}(0)>0$ (a) $t=0$. (b) $t=300 h$. (c) $t=400 h$. (d) $t=520 h$.

estado oscilatório ( Figura 4.8-b). Os diagramas foram gerados calculando-se a norma euclidiana das soluções críticas de (4.38)-(4.39) com a variação de $\lambda_{0}$. Para determinar a estabilidade de cada ramo devemos considerar (4.38) e (4.39) escritas na seguinte forma:

$$
\begin{array}{r}
a_{1}(\eta+1)=f\left(a_{1}(\eta), a_{J-1}(\eta)\right) \\
a_{J-1}(\eta+1)=g\left(a_{1}(\eta), a_{J-1}(\eta)\right)
\end{array}
$$

Um ponto crítico de (4.40)-(4.41) é estável se $\left|\sigma_{s}\right|<1$, onde $\sigma_{s}$ são os autovalores da matriz jacobiana de (4.40)-(4.41) calculada no ponto crítico (ver Mickens [14] pag. 271). O método numérico $(P D)$ será instável se a componente com $s=J-1$ ou $s=1$ for amplificado mais rapidamente que em $(P)$. Desta forma a interação das duas componentes é crucial para aproximação de $(P)$ por $(P D)$. Esta análise mostra um mecanismo direto para tranferência e geração não linear de energia entre a componente mais e menos oscilatória na discretização de $(P D)$. Esta possibilidade, que tem importantes efeitos na estabilidade numérica de $(P D)$, é conseqüência da interação entre a dinâmica fundamental de EDP e o artefato da discretização.

A Figura (4.9) mostra a solução de $(P D) \operatorname{com} f(u)=\operatorname{senh}(u)$. Os 


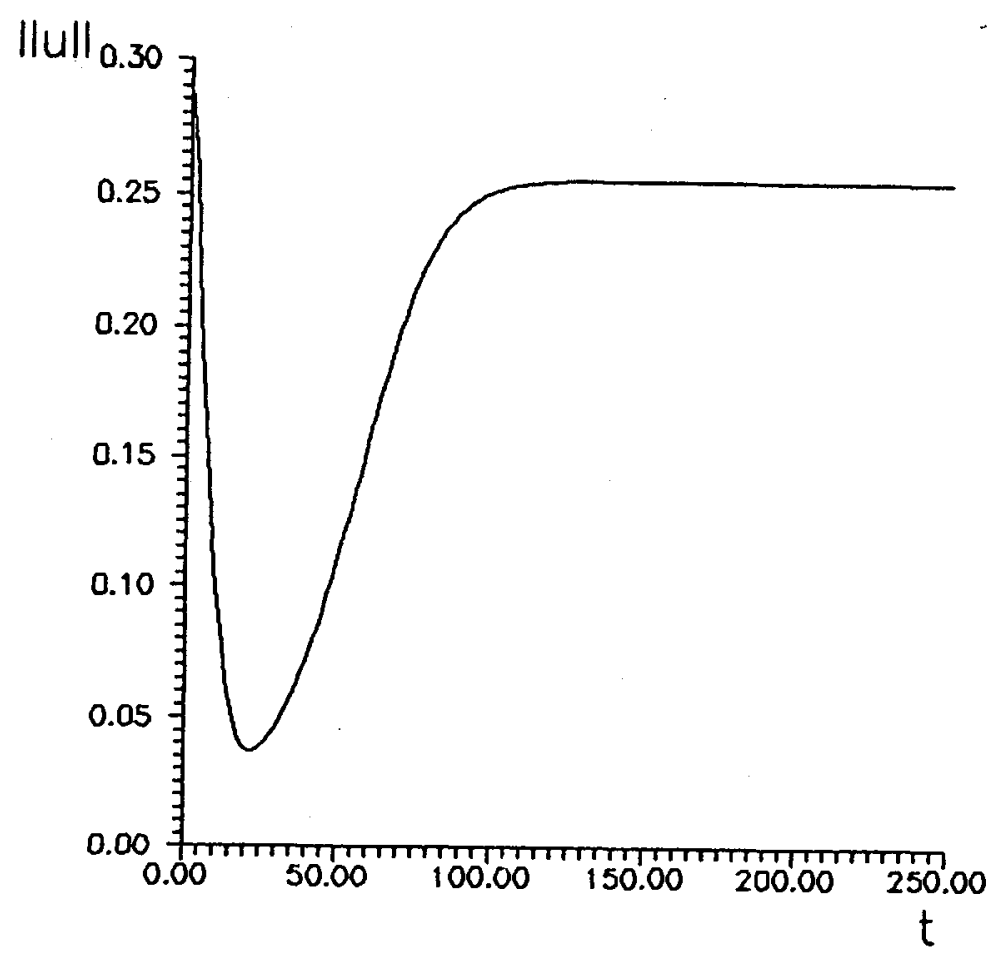

Figura 4.10: Comportamento da norma da solução de $(P D)$ em relação ao tempo para $r<r_{c}, \lambda<\lambda_{c}$ e $f^{\prime \prime \prime}(0)>0$.

parâmetros foram escolhidos tais que $k=0.05, \lambda=10.0\left(>\lambda_{c}\right)$ e $r>r_{c}$. A condição inicial é regular o suficiente para que a excitação inicial de $a_{J-1}$ seja muito pequena em relação a de $a_{1}$. Inicialmente a solução evolui de forma estável, enquanto a componente mais oscilatória $a_{J-1}$ é estimulada pelos efeitos lineares e por intermédio da interação com $a_{1}$ ( ver as equações (4.38) e (4.39) e Figura 4.9-b). Após um período de tempo, $a_{J-1}$ " domina " a solução, como mostra Figura 4.9-c. De qualquer forma, existe uma tranferência e geração de energia em $a_{1}$ e após o perfil da Figura 4.9-d a solução atinge "overflow".

A Figura 4.10 mostra o comportamento da norma da solução de $(P D)$ com $f(u)=u+u^{3}$. Os parâmetros são escolhidos tais que $k=0.05, \lambda=$ $9.7\left(<\lambda_{c}\right)$ e $r<r_{c}$. A condição inicial é formada pelas componentes mais e menos oscilatórias $\left(u_{o}(x)=0.01 * \operatorname{sen}(19 \pi x)+0.1 * \operatorname{sen}(\pi x)\right)$. A instabilidade linear amplifica a componente mais instável até que a não linearidade balanceia a instabilidade, e o equilibrio é atingido. 


\subsection{Bifurcação no Infinito}

Nesta seção analisaremos $(P D)$ no caso (II) onde $f^{\prime}(0)=0$. É possível discutir este problema da perspectiva da seção 3.6, ou seja, bifurcações de soluções estáticas através de $\lambda=\infty$. Consideremos $\theta=1 / 2$ e vamos discutir bifurcações das soluções $n$-dependentes através de $r=\infty$. Sendo $\theta=1 / 2$ de $(P D)$ temos:

$$
\left\{\begin{array}{l}
2\left(u_{j}^{n+1}-u_{j}^{n}\right)=r \delta_{x}{ }^{2}\left(u_{j}^{n+1}+u_{j}^{n}\right)+\lambda h\left[f\left(u_{j}^{n+1}\right)+f\left(u_{j}^{n}\right)\right] \\
u_{0}^{n}=u_{J}^{n}=0 \\
u_{j}^{0}=u_{0}(j k) j=1,2, \ldots, J-1
\end{array}\right.
$$

Linearizando sobre $u_{j}^{n} \equiv 0$ e resolvendo o problema resultante obtem-se:

$$
v_{j}^{n+1}=\sum_{s=1}^{J-1} A_{s} \xi_{s}^{n} \operatorname{sen}\left(\frac{s \pi j}{J}\right)
$$

onde os $A_{s}$ são determinados pela condição inicial e os $\xi_{s}$ são obtidos de (4.7) $\operatorname{com} \theta=1 / 2$, ou seja:

$$
\xi_{s}=\frac{1-2 r \operatorname{sen}^{2}\left(\frac{s \pi}{2 J}\right)}{1+2 r \operatorname{sen}^{2}\left(\frac{s \pi}{2 J}\right)}
$$

Com isto temos que $\left|\xi_{s}\right|<1$ para $s=1,2, \ldots, J-1$. Desta forma $(P D)$ é numericamente estável de acordo com a teoria linear, para todos os valores de $r$, pois $\left|\xi_{s}\right|<1$ para $s=1,2, \ldots, J-1$. De qualquer modo quando $r \rightarrow \infty$ temos que $\left|\xi_{s}\right| \rightarrow 1$. Isto sugere que $r=\infty$ é um candidato a ponto de bifurcação em (4.42). Podemos fazer $r \rightarrow \infty$ tomando $k=h r_{0}{ }^{-1 / 2}$ e fazendo $h \rightarrow 0$. Neste caso (4.43) pode ser escrita na seguinte forma:

$$
\xi_{s}=\frac{h-2 r_{0} \operatorname{sen}^{2}\left(\frac{s \pi h}{2 r_{0} 1 / 2}\right)}{h+2 r_{0} \operatorname{sen}^{2}\left(\frac{s \pi h}{2 r_{0}^{1 / 2}}\right)}
$$

Quando $h \rightarrow 0$ temos que $\xi_{s} \rightarrow 1$, se $s$ satifaz $s h \ll 1$, caso contrário $\xi_{s} \rightarrow-1$. Desde que a taxa de crescimento aproxima-se de valores neutros em $n$, deduzimos que $h=0$ é um candidato a ponto de bifurcação.

Como no caso contínuo, uma análise não linear fraca não será produtiva, pois neste caso todas as componentes são aproximadamente neutras . De qualquer modo a discussão sugere que de alguma forma bifurcam soluções $n$-dependentes através de $r=\infty$. Isto volta a sugerir que alguma forma de instabilidade de amplitude finita é possivel, visto que as soluções bifurcantes no infinito certamente repelem dados iniciais numa vizinhança. É certo que, na prática, não é sensato empregar o método com $r \gg 1$. Todavia, se o ramo da solução bifurcante através de $r=\infty$ estende para valores moderados de $r$, então a dinâmica de (4.42) é afetada desde que a instabilidade de amplitude finita possa ocorrer. 


\section{Capítulo 5}

\section{Conclusão}

Nos capítulos 1 e 2 introduzimos o problema parabólico e os métodos de discretização por diferenças finitas. No capítulo 3 analisamos o problema de Reação-Difusão (problema $(P)$ ) através da teoria da bifurcação local, estabilidade linear e estabilidade não linear fraca. No capítulo 4 analisamos a discretização do problema de Reação-Difusão (problema $(P D)$ ) de forma análoga a feita no capítulo 3.

O nosso objetivo foi descrever a manifestação da instabilidade numérica nos problemas onde o mecanismo de dissipação é balanceado por uma não linearidade. Temos analisado $(P)$ e $(P D)$ numa vizinhança da solução trivial, e os pontos importantes a serem destacados são:

(1) A taxa de crescimento nos problemas linearizado, $\sigma$ em $(P)$ e $\xi$ em $(P D)$, são necessariamente reais (Teoremas 3.3.1 e 4.2.1).

(2) Bifurcação e mudança de estabilidade na solução trivial estão associadas com $\operatorname{Re}(\sigma)=0$ em $(P)$ e $|\xi|=1$ em $(P D)$.

(3) Dos pontos onde bifurcam soluções estáticas em $(P)$ temos que $\sigma=0$, o que corresponde a $\xi=1 \mathrm{em}(P D)$. Obtemos boas aproximações dos pontos de bifurcação e das amplitudes das soluções bifurcantes de $(P)$ através de $(P D)$, quando $\sqrt{\lambda} / \pi \ll J$.

(4) Os pontos onde bifurcam soluções periódicas em $(P D)$ temos $\xi=-1$, os quais não têm correspondentes em $(P)$ ( $\sigma$ é real). Este comportamento é produto da discretização, e está associado com a instabilidade numérica em $(P D)$. 
(5) A manifestação da instabilidade numérica depende crucialmente das propriedades das orbitas periódicas bifurcantes. Se a orbita repele os dados iniciais numa vizinhança, então a instabilidade numérica se manifesta com o crescimento ilimitado de um harmônico oscilatório, caso contrário instabilidade de amplitude finita pode ocorrer.

(6) A existência da componente mista demostra que o início da instabilidade está associado com bifurcação secundária de órbitas periódicas. A direta interação entre o crescimento do harmônico de menor oscilação $(\operatorname{sen}(\pi x)$ ) presente em $(P)$ e $(P D)$, e do harmônico de maior oscilação produzido pela discretização $(\operatorname{sen}((J-1) \pi x))$, é fundamental para a estabilidade numérica não linear ( seção 4.4 caso (c) ).

Duas observações sobre os resultados aqui obtidos se fazem necessárias. Primeiro, nossa análise está fortemente baseada na simplificação dos problemas $(P)$ e $(P D)$ numa vizinhança da solução trivial. Todavia, a bifurcação secundária de uma órbita periódica, que desestabiliza numericamente o problema, ocorre em qualquer vizinhança de uma solução estatica de $(P)$. Segundo, nossa análise é realizada escolhendo $r \approx r_{c}$, sendo que na prática é sensato escolher $r<r_{c}$.

Em problemas mais complicados que $(P)$, o limite da estabilidade linear pode não ser conhecido explicitamente. Assim é importante saber reconhecer as diversas manifestações da instabilidade numérica em problemas não lineares. A análise dos métodos numéricos será certamente beneficiada através da interação com a Teoria da Bifurcação e dos Sistemas Dinâmicos. É importante a comparação do diagrama de bifurcação do problema discreto, com o diagrama do problema contínuo. $\mathrm{Na}$ prática isto significa examinar os resultados numéricos para um domínio dos parâmetros físicos, ao contrário da análise clássica que considera a convergência para um conjunto fixo de parâmetros. Isto revela escalas criticas de natureza física dos parâmetros e da discretização do problema. Em resumo, é indispensável considerar o método numérico como um "sistema dinâmico" e comparar o fluxo gerado numericamente com a essência do problema contínuo.

Nos exemplos aqui apresentados, os dados numéricos foram gerados através de implementação na linguagem Turbo Pascal V. 3.01A, e os gráficos foram gerados através do Grapher V. 2.0.

Neste trabalho restringimos nossa análise a discretização do problema $(P)$, pelos métodos de Crank-Nicolson, considerando duas classes de não linearidade $f(u)$. Assim novas pesquisas podem ser realizadas procurando generalizar o problema $(P)$ e a não linearidade $f(u)$, como também discretizar $(P)$ por outros métodos. 


\section{Bibliografia}

[1] BOHL, E. Discrete versus continuous models for dissipative systems. In: KUPPER, T.; MITTELMANN, H. D.; WEBER, H.; Numerical Methods for Bifurcation Problems. Boston, Birkhäuser, 1984. p. $68-78$.

[2] BRITTON, N. F. Reaction-Diffusion Equations and Applications to Biology. New York, Academic Press, 1986.

[3] CHOW, S. N.; HALE, J. Methods of Bifurcation Theory. Berlin, Springer, 1982.

[4] CONWAY, E.; HOFF, D.; SMOLLER, J. Large time behavior of solutions of systems of nonlinear reaction-diffusion equations. SIAM J. Appl. Math., V. 35, N. 1,p. 1-16, 1978.

[5] FIGUEIREDO, D. G. Análise de Fourier e Equações Diferenciais Parciais. Rio de Janeiro, IMPA, 1977.

[6] FLETT, T. M. Differential Analysis Lodon, Cambridge, 1980.

[7] HENRICI, P. Elements of Numerical Analisis. New York, Wiley, 1964.

[8] HOFF, D. Stability and convergence of finite difference methods for systems of nonlinear reaction-difusion equations. SIAM J. Numer. Anal., V. 15, N. 6, p. 1161 - 71, 1978.

[9]. IÓRIO, R.; IÓRIO, V. M. Equações Diferenciais Parciais: 'Uma Introdução. Rio de Janeiro, IMPA, 1988.

[10] IOOSS, G.; JOSEPH, D. D. Elementary Stability and Bifurcation Theory. New York, Springer - Verlag, 1980.

[11] KIELHÖFER, H. On the Lyapunov-Stability of stationary solutions of semilinear parabolic differential equations. J. Differ. Equations, V. 22, N. 1, p. 193-208, 1976. 
[12] LEES, M. A linear three-level difference scheme for quasilinear parabolic equation. Math. Comp., V. 20, N. 1, p. 516-22, 1966.

[13] MATKOWSKY, B. J. A simple nonlinear dynamic stability problem. Bull. Amer. Math. Soc., V. 76, p. 620-25. 1970.

[14] MICKENS, R. E. Difference Equations Theory and Applications. New York, Van Nostrand Reinhold, 1990.

[15] NEWELL, A. C. Finite amplitude instabilities of parcial differential equation. SIAM J. Appl. Math., V. 33, N. 1, p. 133-60, 1985

[16] RABINOWITZ, P. H. Applications of Bifurcation Theory. New York,Academic Press, 1977.

[17] RICHTMYER, R.; MORTON, K. Difference Methods for Initial Value Problems. New York, Intercience, 1967.

[18] ROSEMBLAT, S.; DAVIS, S. H. Bifurcation from infinity. SIAM. J. Appl. Math., V. 37, N. 1, p. 1-19. 1979.

[19] SMITH, G. D. Partial Differential Equation - Finite Difference Methods. Oxford, Clarendon Press, 1985.

[20] SMOLLER, J. Shock Waves and Reaction-Diffusion Equations. New York, Springer, 1980.

[21] SPIEGEL, M. R. Finite Differences and Difference Equations. New York, Mc Graw-Hill, 1976

[22] STUART, A. M. Nonlinear instability dissipative finite difference schemes. SIAM Review, V. 31, N. 2, p. 191-220. 1989

[23] STUART, A. M. A note on high/low wave number interaction in spatially discrete parabolic equations. IMA $J$ Appl. Math., V. 42, N. 1, p. 27-42, 1989.

[24] WEISS, R. Bifurcation in difference approximations to two-point bondary value problems. Math. Comp., V. 29, N. 131, p. 746-60. 1975.

[25] WILlians, W. E. Partial Differential Equations. Oxford,Clarendon, 1980. 